\title{
In-medium loop corrections and longitudinally polarized gauge bosons in high-energy showers
}

\author{
Peter Arnold $^{a}$ and Shahin Iqbal ${ }^{b}$ \\ ${ }^{a}$ Department of Physics, University of Virginia, \\ Charlottesville, Virginia 22904-4714, U.S.A. \\ ${ }^{b}$ National Centre for Physics, Quaid-i-Azam University Campus, \\ Islamabad 45320, Pakistan \\ E-mail: parnold@virginia.edu, smi6nd@virginia.edu
}

ABSTRACT: The splitting processes of bremsstrahlung and pair production in a medium are coherent over large distances in the very high energy limit, which leads to a suppression known as the Landau-Pomeranchuk-Migdal (LPM) effect. We continue study of the case when the coherence lengths of two consecutive splitting processes overlap (which is important for understanding corrections to standard treatments of the LPM effect in QCD), avoiding soft-emission approximations. In this particular paper, we show (i) how the "instantaneous" interactions of Light-Cone Perturbation Theory must be included in the calculation to account for effects of longitudinally-polarized gauge bosons in intermediate states, and (ii) how to compute virtual corrections to LPM emission rates, which will be necessary in order to make infrared-safe calculations of the characteristics of in-medium QCD showering of high-energy partons. In order to develop these topics in as simple a context as possible, we will focus in the current paper not on QCD but on large- $N_{\mathrm{f}}$ QED, where $N_{\mathrm{f}}$ is the number of electron flavors.

KeYwords: Quark-Gluon Plasma, Perturbative QCD

ARXIV EPRINT: 1806.08796 


\section{Contents}

1 Introduction $\quad 1$

1.1 Overview 1

1.2 Overlapping formation times in large- $N_{\mathrm{f}}$ QED 4

$\begin{array}{lll}1.3 & \text { Assumptions and approximations } & 8\end{array}$

$\begin{array}{ll}1.4 & \text { Some qualitative results }\end{array}$

$\begin{array}{lll}1.5 & \text { Outline } & 11\end{array}$

$\begin{array}{lll}1.6 & \text { Reference acronyms } & 11\end{array}$

2 Qualitative differences between QCD and QED 12

$\begin{array}{lll}2.1 & \text { Single splitting } & 12\end{array}$

2.1.1 Formation times 12

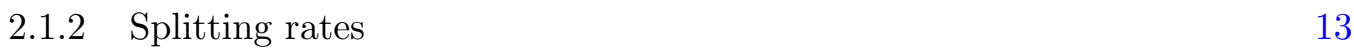

$\begin{array}{ll}\text { 2.1.3 An aside on nearly-democratic splitting } & 14\end{array}$

$\begin{array}{lll}2.2 & \text { Overlapping double splitting } & 14\end{array}$

2.2.1 Review of QCD estimate for $g \rightarrow g g g \quad 14$

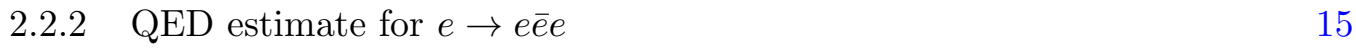

$\begin{array}{ll}2.2 .3 \text { Leading-log formula for } e \rightarrow e \bar{e} e & 16\end{array}$

3 Light cone perturbation theory for overlapping LPM 18

$\begin{array}{ll}3.1 \text { Diagrammatic rules and their translation } & 18\end{array}$

$\begin{array}{lll}3.2 & \text { An implicit approximation } & 22\end{array}$

4 Virtual corrections to single splitting $\quad 24$

4.1 Back-end transformation 24

$\begin{array}{lll}4.2 & \text { Front-end transformations } & 26\end{array}$

$\begin{array}{ll}4.3 \text { The fundamental virtual diagram } & 29\end{array}$

$\begin{array}{lll}4.3 .1 & \text { Overview } & 29\end{array}$

4.3.2 Dimensional regularization: strategy 31

4.3.3 Dimensional regularization: application to $x y y \bar{x} \quad 33$

$\begin{array}{lll}\text { 4.3.4 Renormalization } & 36\end{array}$

4.4 Relation to the $\gamma \rightarrow e \bar{e}$ virtual diagram (r) 38

5 Conclusion $\quad 39$

$\begin{array}{ll}\text { A Summary of results } & 39\end{array}$

A.1 Leading-order single-splitting rates 40

$\begin{array}{ll}\text { A.1.1 } e \rightarrow \gamma e & 40\end{array}$

$\begin{array}{lll}\text { A.1.2 } \gamma \rightarrow e \bar{e} & 40\end{array}$

A.2 Overlap corrections to real double splitting 41

$\begin{array}{lll}\text { A.2.1 Sequential diagrams } & 41\end{array}$

A.2.2 Diagrams with one instantaneous vertex 43 
A.2.3 Diagrams with two instantaneous vertices 44

A.3 NLO corrections to single splitting $e \rightarrow \gamma e \quad 44$

A.4 NLO corrections to single splitting $\gamma \rightarrow e \bar{e}$

B Similarities and dissimilarities with refs. $[23,25] \quad 46$

$\begin{array}{lll}\mathrm{C} & \hat{q} \text { in } \mathrm{QCD} \text { and QED } & 47\end{array}$

$\begin{array}{lll}\text { C.1 } \hat{q} \text { for weakly-coupled systems } & 47\end{array}$

C.2 Small- $x$ logs in QCD 48

$\begin{array}{lll}\text { C.3 The upshot for this paper } & 48\end{array}$

C.4 The Coulomb logarithm in Migdal's QED result 49

$\begin{array}{ll}\text { D Diagrammatic vertex rules } & 50\end{array}$

E Calculation of real double splitting (figure 7) 54

$\begin{array}{lll}\text { E.1 Sequential diagrams } & 55\end{array}$

E.1.1 Generic formulas $\quad 55$

E.1.2 QED formulas for $(\bar{\alpha}, \bar{\beta}, \bar{\gamma}) \quad 56$

E.2 Frequencies $\Omega$ and eigenmodes $\quad 57$

$\begin{array}{ll}\text { E.2.1 3-particle evolution frequency } & 57\end{array}$

$\begin{array}{ll}\text { E.2.2 4-particle evolution frequencies and modes } & 58\end{array}$

$\begin{array}{lll}\text { E.3 Diagrams with instantaneous vertices } & 58\end{array}$

$\begin{array}{ll}\text { E.3.1 The } I \bar{I} \text { diagram } & 59\end{array}$

$\begin{array}{ll}\text { E.3.2 Diagrams with one instantaneous vertex } & 60\end{array}$

F Explicit formulas for evaluation of $x y y \bar{x}$ diagram $\quad 62$

F.1 General formula for $d=2 \quad 62$

F.2 Small $\Delta t$ expansion $\quad 63$

F.2.1 Structure 63

$\begin{array}{ll}\text { F.2.2 Value of } a^{-1^{\top}} \Omega^{2} a^{-1} & 64\end{array}$

F.2.3 Putting it together 66

$\begin{array}{lll}\text { F.3 Needed generalizations to } d=2-\epsilon & 66\end{array}$

$\begin{array}{lll}\text { F.4 The subtraction } \mathcal{D}_{2}(\Delta t) & 68\end{array}$

$\begin{array}{lll}\text { F.4.1 Combination with } \mathbb{I} & 68\end{array}$

$\begin{array}{lll}\text { F.4.2 The subtracted piece } & 69\end{array}$

$\begin{array}{lll}\text { F.5 Integration over } y_{e} & 69\end{array}$

$\begin{array}{lll}\text { F.6 Renormalization } & 70\end{array}$

$\begin{array}{lll}\text { F.7 } & \hat{q} \text { and dimensional regularization } & 71\end{array}$

G The integral II $\quad 71$

G.1 Perturbative treatment of $O\left(M \Omega^{2} \Delta t B^{2}\right)$ corrections to the exponent 71

$\begin{array}{lll}\text { G.2 Units } & 72\end{array}$

$\begin{array}{lll}\text { G.3 Representation of Bessel functions } & 72\end{array}$

$\begin{array}{ll}\text { G.4 Doing the } \boldsymbol{B} \text { integrals } & 73\end{array}$ 
$\begin{array}{lll}\text { G.5 Doing the } \Delta t \text { integral } & 74\end{array}$

$\begin{array}{lll}\text { G.6 } & \text { Evaluating } \mathbb{I}_{\text {regular }} & 75\end{array}$

$\begin{array}{lll}\text { G.7 } & \text { Evaluating } \mathbb{I}_{\text {div }} & 76\end{array}$

G.8 Final result for $\mathbb{I}$ for $M>0 \quad 77$

$\begin{array}{lll}\text { G.9 Generalization to include } M<0 & 78\end{array}$

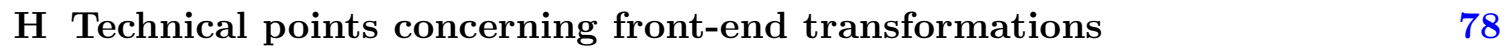

$\begin{array}{lll}\text { H.1 Branch cuts for the transformation }(\mathrm{k}) \rightarrow(\mathrm{r}) & 78\end{array}$

H.2 Front-end transformations in $d=2-\epsilon$ dimensions $\quad 80$

H.2.1 Transformation of leading-order rates 80

$\begin{array}{ll}\text { H.2.2 Transformation of NLO rates } & 81\end{array}$

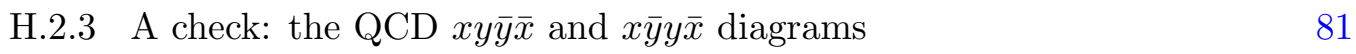

\section{Introduction}

\subsection{Overview}

When passing through matter, high energy particles lose energy by showering, via the splitting processes of hard bremsstrahlung and pair production. At very high energy, the quantum mechanical duration of each splitting process, known as the formation time, exceeds the mean free time for collisions with the medium, leading to a significant reduction in the splitting rate known as the Landau-Pomeranchuk-Migdal (LPM) effect [1-3]. ${ }^{1} \mathrm{~A}$ long-standing problem in field theory has been to understand how to implement this effect in cases where the formation times of two consecutive splittings overlap.

Let $x$ and $y$ be the longitudinal momentum fractions of two consecutive bremsstrahlung gauge bosons. In the limit $y \ll x \ll 1$, the problem of overlapping formation times has been analyzed at leading logarithm order in refs. [4-6] in the context of energy loss of highmomentum partons traversing a QCD medium (such as a quark-gluon plasma). Together with Chang, we subsequently developed and implemented field theory formalism needed for the more general case where $x$ and $y$ are arbitrary [7-10]. We used the formalism to calculate the LPM interference effects on real double gluon bremsstrahlung $g \rightarrow g g g$ in medium, given by the processes depicted in figure 1, in the high-energy limit. [For the sake of simplicity, that specific calculation also made other simplifying approximations by taking the multiple scattering $(\hat{q})$ limit and the large- $N_{\mathrm{c}}$ limit.] But that calculation was incomplete for two reasons.

First, the calculations of refs. [7-10] only included gluons that were transversely polarized. But the polarization of the intermediate-state gluon in the first diagram of figure 1

\footnotetext{
${ }^{1}$ Refs. [1, 2] are also available in English in L. Landau, The Collected Papers of L.D. Landau, Pergamon Press, New York (1965).
} 


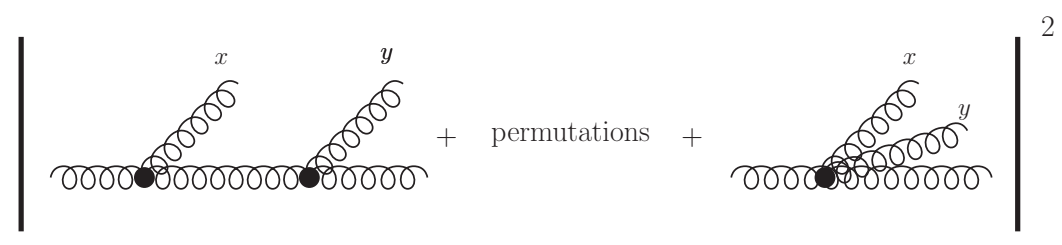

Figure 1. Real double gluon bremsstrahlung processes $g \rightarrow g g g$ considered in the analysis of overlapping formation time effects at high energy in refs. [7-10]. Only the high energy particles are shown: their many interactions with the medium are not shown explicitly. $x$ and $y$ represent the momentum fractions (relative to the initial particle) of two of the daughters of this splitting process.

does not have to be transverse: a full calculation should include the effects of the longitudinal polarization as well. ${ }^{2}$ It has long been known how to integrate out the effects of longitudinal polarizations and find the effective theory of the transverse polarizations, which is the program of Light Cone Perturbation Theory (LCPT) [11-13]. ${ }^{3}$ In LCPT, where light-cone gauge $A^{+}=0$ is used, the longitudinal polarization gives rise to an interaction that is instantaneous in light-cone time $x^{+} \equiv x^{0}+x^{z}$ (similar to how in Coulomb gauge the non-transverse polarization $A^{0}$ of the gauge field responds to sources instantaneously in normal time $x^{0}$, even though physical quantities ultimately do not). In LCPT, the longitudinal polarization can be integrated out to yield a Hamiltonian that is supplemented by just an extra 4-point interaction among the fields. The result is to add one extra diagram (and its permutations), figure 2, to the sum of diagrams depicting the amplitude in figure 1. In figure 2, the bar across the intermediate gluon line indicates that it is an (integrated-out) longitudinally polarized gluon. That gluon line is drawn vertically to emphasize that it represents an instantaneous interaction in the context of time $\left(x^{+}\right)$-ordered perturbation theory, with $x^{+}$running from left to right in the figure. One goal of this paper is to include this type of process into our earlier calculations of the effect of overlapping formation times on sequential bremsstrahlung.

A calculation of in-medium real double bremsstrahlung $g \rightarrow g g g$ is not enough, however, to study the effects on in-medium energy loss and the characteristics of in-medium

\footnotetext{
${ }^{2}$ Some historical explanation: longitudinal polarizations were left out of our earlier analysis of refs. [7-10] because of an unstated and incorrect assumption. Consider the two consecutive vertices appearing in the first diagram of figure 1. Our interest is in the case where the formation times associated with these vertices overlap, in which case the characteristic scale of the time separation of those vertices (and so also their spatial separation in the direction of motion $z$ ) is of order the formation time. That formation time is parametrically large in the large-energy limit $\left(t_{\text {form }} \sim \sqrt{E / \hat{q}}\right.$, ignoring dependence on daughter momentum fractions). The effects of longitudinal polarizations do not extend over large distances, and so ref. [7] implicitly ignored them. But that's not a valid argument that the effects of nearly-coincident gluon emissions can be ignored, as was made clear in our own calculations by the subsequent calculation in ref. [10] of the 4-point vertex contributions represented by the last diagram of figure 1. It was not until we started computing virtual corrections (discussed in a moment) that we clearly realized our mistake in ignoring intermediate longitudinal polarizations.

${ }^{3}$ For readers not familiar with time-ordered LCPT who would like the simplest possible example of how it reassuringly reproduces the results of ordinary Feynman diagram calculations, we recommend section 1.4.1 of Kovchegov and Levin's monograph [14]. For some less-simple examples, see also ref. [15].
} 


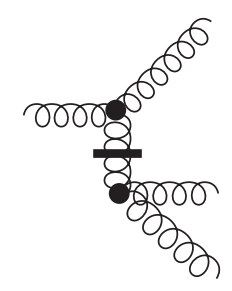

Figure 2. The contribution to $g \rightarrow g g g$ due to longitudinal polarization of the intermediate gluon line (represented by a bar across the line). This process must be added to the $g \rightarrow g g g$ amplitude of figure 1 if the intermediate lines in figure 1 are summed only over transverse polarizations as in refs. $[7-10]$.

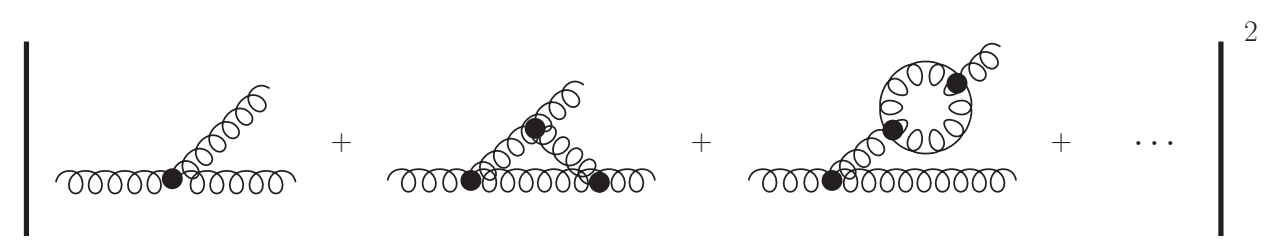

Figure 3. Examples of one-loop virtual corrections to single bremsstrahlung $g \rightarrow g g$.

shower development. Crudely analogous to what happens for vacuum bremsstrahlung in QED, there are infrared divergences whose treatment at this order requires additionally computing virtual corrections to single bremsstrahlung $g \rightarrow g g$, such as depicted by the diagrams in figure 3 . The second goal of this paper is to show how to calculate such loop corrections (without soft gluon approximations) in the context of finding the effects of overlapping formation times on energy loss and shower development. A new technical feature, compared to our calculations of $g \rightarrow g g g$, is that loop calculations require UV regularization and renormalization. In particular, one of the effects of loops (besides mitigating certain infrared divergences of the $g \rightarrow g g g$ calculation) will be to replace the coupling $\alpha_{\mathrm{S}}$ in the leading-order single-bremsstrahlung process $g \rightarrow g g$ by the running coupling $\alpha_{\mathrm{s}}\left(Q_{\perp}\right)$ evaluated at a characteristic transverse momentum scale of the LPM-modified bremsstrahlung process.

In this particular paper, we work out all of these issues for a warm-up theory: QED in the large- $N_{\mathrm{f}}$ limit, where $N_{\mathrm{f}}$ is the number of electron flavors. The large- $N_{\mathrm{f}}$ limit is taken just to reduce the complexity of the calculations and so streamline the presentation of the important developments. The method itself should be straightforwardly generalizable to the QCD analysis of the LPM effect in figures 1-3 (at least in the large- $N_{\mathrm{c}}$ limit of refs. [7-10]), but we leave that QCD calculation for the future.

There are several aspects of this paper that are related to recent work by Beuf $[22,23]$ and Hänninen, Lappi, and Paatelainen [24, 25] on next-to-leading-order deep inelastic scattering (NLO DIS). Here, making use of LCPT, we will study the combination of $1 \rightarrow 3$ splitting $e \rightarrow e \bar{e} e$ and UV-renormalized one-loop corrections to $1 \rightarrow 2$ splitting $(e \rightarrow \gamma e$ or $\gamma \rightarrow e \bar{e}$ ) in the presence of a thick medium (though the formalism we use can in principle handle more general situations). In NLO DIS, Beuf and Hänninen et al. instead used 


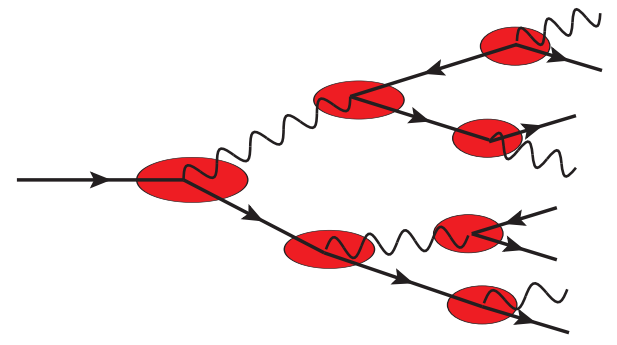

(a)

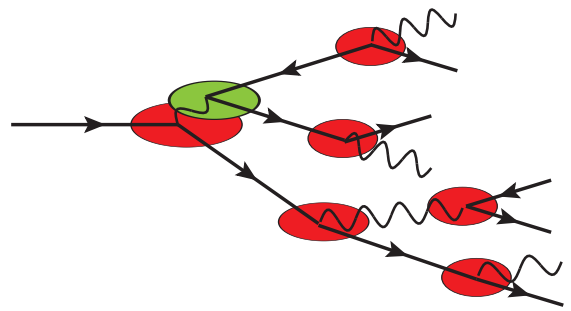

(b)

Figure 4. Schematic picture of in-medium QED showers of an initial high energy electron. The ovals represent LPM formation lengths for each splitting. In (a), splittings are independent and do not interfere with each other. In (b), two splittings have overlapping formation lengths and so may not be treated as independent. Showering is extremely collinear in the high energy limit, but the transverse directions in these schematic drawings have been drastically magnified for the purpose of illustration.

LCPT to study the combination of $1 \rightarrow 3$ splitting $\gamma^{*} \rightarrow q \bar{q} g$ and UV-renormalized one-loop corrections to $1 \rightarrow 2$ splitting $\left(\gamma^{*} \rightarrow q \bar{q}\right)$ in the presence of an extremely thin medium. We briefly comment further on the similarities and dissimilarities in appendix B.

The goal of this paper is to develop calculational methods. We leave discussion of application of the results to computing IR-safe characteristics of energy loss and shower development to a later paper [16].

\subsection{Overlapping formation times in large- $N_{\mathrm{f}}$ QED}

Figure 4 depicts the showering of a high-energy particle in a medium. For simplicity of discussion, let's focus for the moment on roughly-democratic splitting processes, meaning that neither daughter of any splitting is soft (i.e. neither has very small energy compared to the parent). Let's also first discuss the case of QED with only one electron flavor $\left(N_{\mathrm{f}}=1\right)$. If the coupling associated with the splitting vertices is small, then there is a hierarchy of scale in figure 4 between (i) the formation length $l_{\text {form }}$ associated with each splitting (denoted by the length of the ovals) and (ii) the mean free path $l_{\text {rad }}$ between consecutive roughly-democratic splittings. Parametrically,

$$
l_{\mathrm{rad}} \sim \frac{l_{\text {form }}}{\alpha} .
$$

A rough mnemonic for this result is that each formation length of media traversed offers one opportunity for splitting, with probability of order $\alpha$. On average it then takes $\sim 1 / \alpha$ such opportunities to radiate.

Because of this scale hierarchy, the probability for two consecutive splittings to have overlapping formation times, as depicted in figure $4 \mathrm{~b}$, is suppressed by a factor of $\alpha$. To leading order in $\alpha$, one may treat the splittings in showers as non-overlapping, as depicted in figure 4a, and so may treat the probabilities of each splitting in the shower as independent. The goal of the current program is to understand how to calculate (beyond 


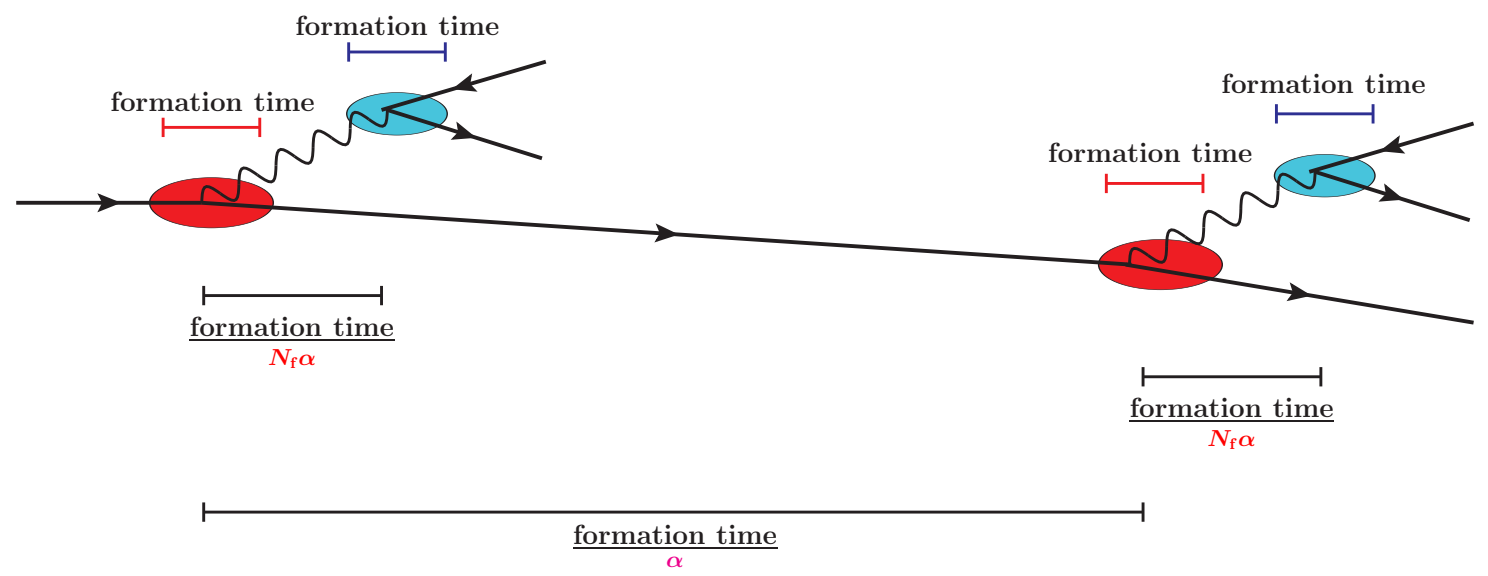

Figure 5. Parametric summary of relative size of typical formation lengths and distances between splittings for large- $N_{\mathrm{f}}$ QED when $\alpha \ll N_{\mathrm{f}} \alpha \ll 1$. In this picture, splittings are assumed to be roughly democratic, i.e. we are not depicting the case of soft daughters. We have not bothered to show the part of the shower representing the subsequent evolution of pair-produced electrons and positrons, but they behave similarly to the evolution of the original electron.

soft-emission approximations) the $\alpha$-suppressed effect of overlapping formation times, such as the overlapping pair of splittings in figure $4 \mathrm{~b}$.

So far, the qualitative discussion has been the same as the discussion for QCD in the introduction of ref. [7]. But now consider the large- $N_{\mathrm{f}}$ limit, which will reduce the number of calculations needed in this paper. If the properties of the medium are held constant, then $N_{\mathrm{f}}$ does not affect the rate for bremsstrahlung $e \rightarrow \gamma e$. Formation times are also not affected. But the rate for pair production $\gamma \rightarrow e \bar{e}$ is proportional to $N_{\mathrm{f}}$, and so the mean free path for pair production is smaller than for bremsstrahlung by a factor of $N_{\mathrm{f}}$. The hierarchy of scales relevant to typical showering is then summarized by figure 5 , assuming that $N_{\mathrm{f}}$ is large but $N_{\mathrm{f}} \alpha$ is still small: ${ }^{4}$

$$
\alpha \ll N_{\mathrm{f}} \alpha \ll 1
$$

The probability that two of the closer splittings in the figure $(e \rightarrow \gamma e \rightarrow e \bar{e} e)$ might overlap will be of order $N_{\mathrm{f}} \alpha$. That's overlap of (i) bremsstrahlung with (ii) the subsequent pair production later initiated by the bremsstrahlung photon. The chance of any other type of overlap, such as two consecutive bremsstrahlung processes $e \rightarrow \gamma e \rightarrow \gamma \gamma e$, is only of order $\alpha$ and so is parametrically less likely in the large- $N_{\mathrm{f}}$ limit. So, to get the dominant correction due to overlapping formation lengths in this problem under the formal assumption (1.2), the only type of overlap we need to compute is the type shown in figure 6 (plus related virtual processes).

The interference diagrams needed to calculate the effect of overlapping formation times in large- $N_{\mathrm{f}} \mathrm{QED}$ are shown in figures 7-9. These diagrams are drawn using the conventions

\footnotetext{
${ }^{4}$ We will not attempt it here, but it may well be possible to sum all orders of $N_{\mathrm{f}} \alpha$ for $\alpha \ll 1$, similar in spirit to the analysis of transport and pressure in refs. [17] and [18, 19].
} 


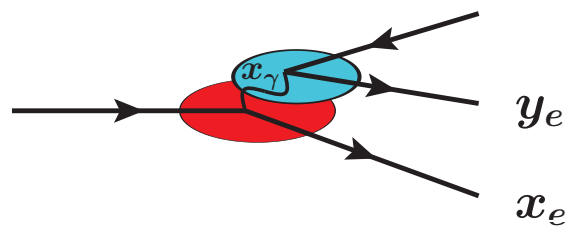

Figure 6. The dominant type of overlap correction when $\alpha \ll N_{\mathrm{f}} \alpha \ll 1$. (Also shown here is the convention we will use later to label the longitudinal momentum fractions $x_{e}$ and $y_{e}$ of the two final-state electrons relative to the initial electron. The corresponding fraction of the intermediate photon is $x_{\gamma}=1-x_{e}$.)

Diagrams with only transverse intermediate photons:

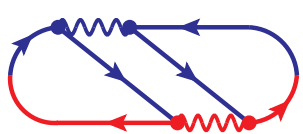

(a)

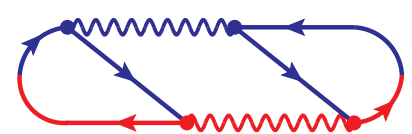

(b)

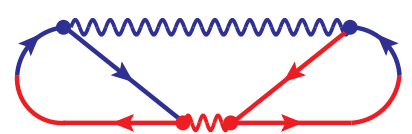

(c)

Diagrams including longitudinal intermediate photons:

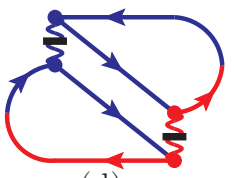

(d)

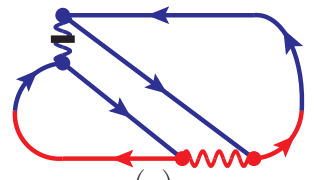

(e)

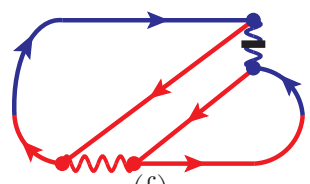

(f)

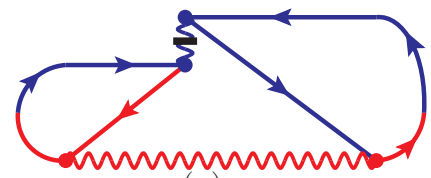

(g)

Figure 7. Time-ordered interference diagrams for $e \rightarrow e \bar{e} e$ in large- $N_{\mathrm{f}}$ QED. As in refs. [7, 8], blue represents a contribution to the amplitude and red represents a contribution to the conjugate amplitude. As in the other figures of this paper, repeated interactions with the medium are present but not explicitly shown. The complex conjugates of the above interference diagrams should also be included by taking $2 \operatorname{Re}[\cdots]$ of the above.

of refs. [7, 8] and represent contributions to the rate for the process. Each diagram shows the product of a term in the amplitude for the process (colored blue in the diagram) times a term in the conjugate amplitude (colored red). The diagrams are time-ordered (more precisely in this paper, light-cone time ordered), with time running from left to right. We will only consider rates which are integrated over the transverse momenta of the final particles, which allows one to ignore the evolution of any final-state particle after it has been emitted in both the amplitude and conjugate amplitude. ${ }^{5}$ So, for instance, the diagrams of figure $7 \mathrm{a}$ and $7 \mathrm{~g}$ represent the $\left(\boldsymbol{p}_{\perp}\right.$-integrated $)$ interferences of figure 10 .

We will see later that the results for most of the virtual diagrams can be related to results for $e \rightarrow e \bar{e} e$ (figure 7 ), or to each other. There will only be one quintessential virtual diagram that we will have to compute from scratch, which will turn out to be the boxed diagram shown in figure 8k. That diagram, and the related diagram of figure 9r, contain the only true UV divergence in these large- $N_{\mathrm{f}}$ calculations ${ }^{6}$ and will be the diagrams responsible for the usual renormalization of the QED coupling $\alpha$.

\footnotetext{
${ }^{5}$ See the discussion in section IV.A of ref. [7] and appendix F of ref. [8].

${ }^{6}$ One simplification of the large- $N_{\mathrm{f}}$ limit is that we do not have one-loop fermion self energies nor one-loop vertex corrections, and so we need not also compute the divergent diagrams associated with those.
} 


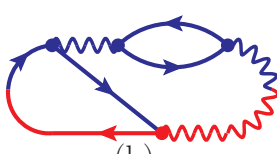

(h)

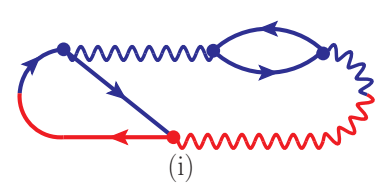

(i)

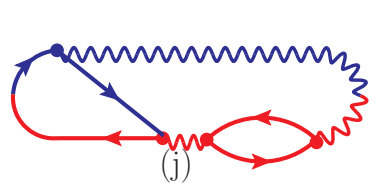

(j)
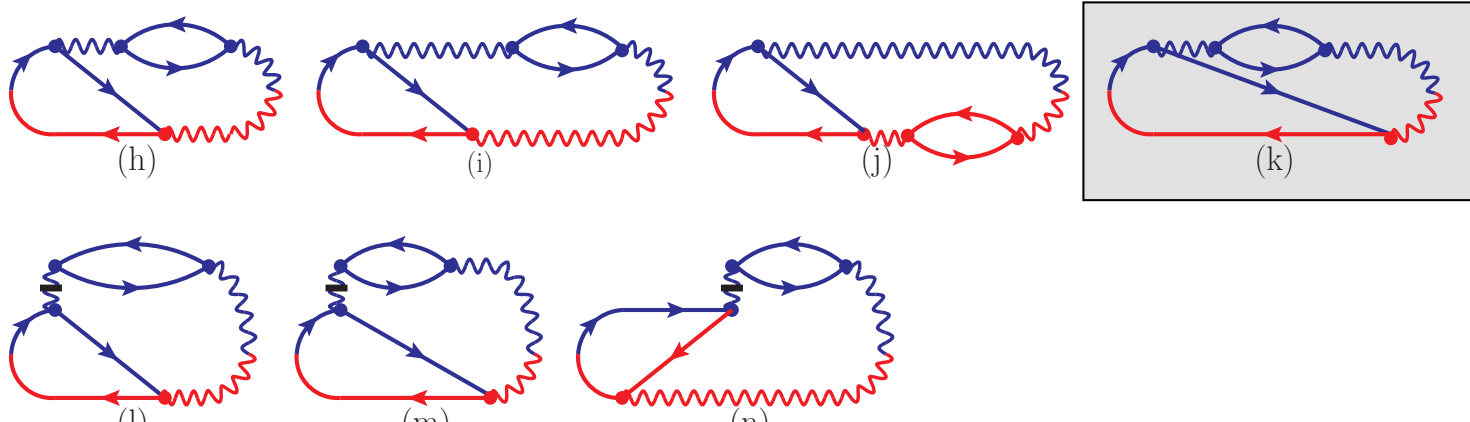

$(\mathrm{m})$

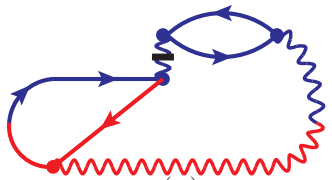

(n)

Figure 8. Time-ordered interference diagrams for the virtual correction to $e \rightarrow \gamma e$ in large- $N_{\mathrm{f}}$ QED. The boxed diagram is the only one whose result cannot be simply related to one of the $e \rightarrow e \bar{e} e$ diagrams of figure 7 . Again, complex conjugates should be included by taking $2 \operatorname{Re}[\cdots]$.
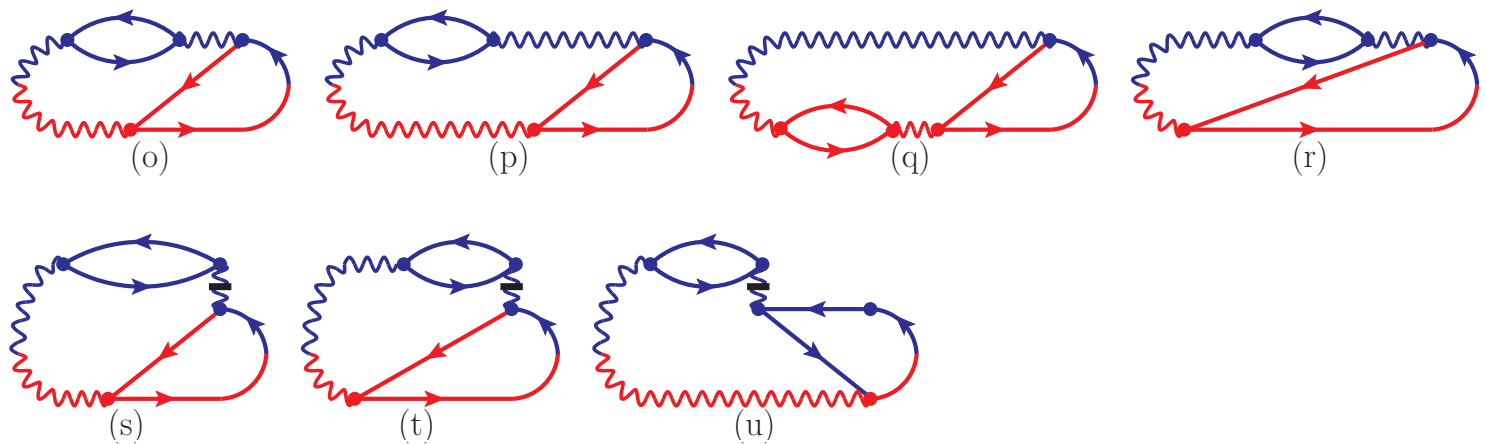

(u)

Figure 9. Time-ordered interference diagrams for the virtual correction to $\gamma \rightarrow e \bar{e}$ in large- $N_{\mathrm{f}}$ QED. All of these diagrams can be related to one of the diagrams of figures 7 or 8 .

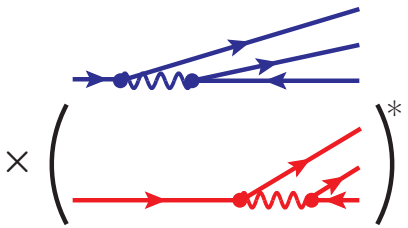

(a)

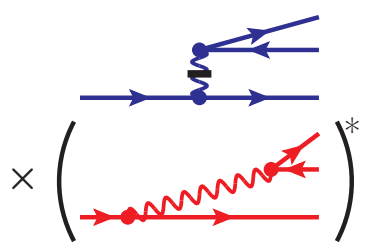

(g)

Figure 10. The interferences represented by figures $7 \mathrm{a}$ and $7 \mathrm{~g}$. These interferences are still timeordered. For example, in (a), the vertex times in the conjugate amplitude are restricted to be larger than those in the amplitude, as depicted.

In the approach of our earlier work [7-10], inspired by Zakharov's treatment of the LPM effect $[20,21]$, we interpret the interference diagram of figure $7 \mathrm{a}$, for instance, as the evolution of an initial $e \bar{e}$ pair, where the $e$ represents the initial electron in the amplitude and the $\bar{e}$ represents the same particle in the conjugate amplitude. In this language, the time evolution of figure 7a is interpreted, as shown in figure 11a, as a phase of 3-particle evolution ( $\gamma e$ in the amplitude and $\bar{e}$ in the conjugate amplitude), followed by 4 -particle evolution (eee in the amplitude and $\bar{e}$ in the conjugate amplitude), followed by 3-particle evolution 


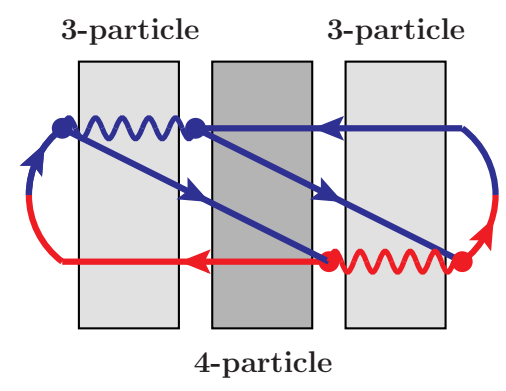

(a)

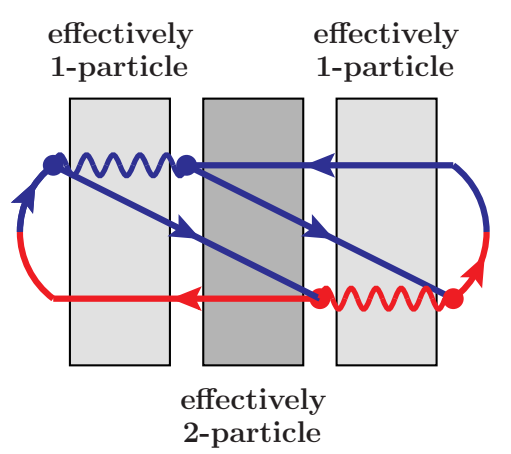

(b)

Figure 11. (a) Interpretation of the interference diagram as simultaneous evolution in time of the particles in the amplitude and conjugate amplitude (all interacting with the medium, and averaged over the randomness of the medium). The labels denote how many high-energy particles need to be evolved between splitting vertices. (b) Figure (a) relabeled according to the effective number of particles needed after symmetries are used to reduce the problem [7].

(e $\bar{e}$ in the amplitude and $\gamma$ in the conjugate amplitude). We then used symmetries of the problem to replace each medium-averaged $N$-particle evolution problem by an effectively $(N-2)$-particle evolution problem, ${ }^{7}$ as summarized in figure $11 \mathrm{~b}$. One of our tasks in the current paper will be to translate the LCPT diagrammatic rules for longitudinal photon interactions into corresponding rules within our framework.

Before going forward, we should mention that formally there are some additional virtual diagrams in Light Cone Perturbation Theory, which will be shown and discussed later (figure 23) but which are negligible in the high-energy limit provided we use dimensional regularization.

\subsection{Assumptions and approximations}

As with the earlier work in refs. [7-10], the formalism we will present is quite general, but for now we will implement it in simple cases that make the actual calculations much easier.

First, we will assume that the medium is large, static, and uniform. More precisely, we approximate the medium as (statistically) uniform and unchanging over the scale of formation lengths/times.

\footnotetext{
${ }^{7}$ For a full discussion of this reduction, see section III of ref. [7]. Let $z$ be the direction of motion of the initial particle. In the high-energy limit, the propagation of the $N$ particles can be formulated as a twodimensional non-relativistic Schrödinger problem in the transverse $(x y)$ plane, with longitudinal momenta $p_{z i}$ playing the role of the "masses" in the Schrödinger equation. One of the symmetries is transverse translation invariance (over the small transverse distance scales probed by the splitting), which means that one can eliminate one degree of freedom by taking out the uninteresting "center of mass" motion, reducing the problem to effectively $N-1$ (two-dimensional) degrees of freedom. The other symmetry is that of small 3-dimensional rotations which change the direction of the $z$ axis by a very tiny amount (and so preserve $\left|\boldsymbol{p}_{\perp}\right| \ll p_{z}$ for all the particles). For our particular problem, this symmetry allows one to remove one additional degree of freedom. This is not obvious; refer to ref. [7] for details. The specific case of reducing 3 particles to 1 particle was implicit to the analysis of BDMPS [27-29] and Zakharov [20, 21], though presented in different language.
} 
Second, we will make the "multiple scattering approximation" that interactions with the medium can be characterized by the parameter $\hat{q}$ that is often used in discussions of quark-gluon plasmas, and which is the proportionality constant in the relationship $\left\langle Q_{\perp}^{2}\right\rangle=$ $\hat{q} \Delta z$, where $Q_{\perp}$ is the transverse momentum that a high-energy charged particle picks up passing through length $\Delta z$ of the medium. For a variety of reasons, the effective value of $\hat{q}$ can have logarithmic dependence on energy at fixed order in the coupling $\alpha$ that controls high-energy splittings. There are important qualitative differences between QCD and QED, which we briefly review in appendix $\mathrm{C}$, but it is off the main topic of this paper. Here, we will make the approximation that $\hat{q}$ is a constant and assume that one is using a value of $\hat{q}$ appropriate for the overall energy scale of the initial particle.

\subsection{Some qualitative results}

This paper further develops methods for next-to-leading order calculations of the LPM effect and gives analytic results for the case of large- $N_{\mathrm{f}}$ QED. The details of the full analytic results are complicated enough that we will not present them here in the introduction. And we are mostly leaving numerical analysis and application of those results to a later paper. But there are two features of our results which we will present here.

The first feature regards the appropriate choice of renormalization scale for the factor of $\alpha$ that controls the cost of high-energy splitting and determines the overall importance of overlapping formation times in showering - that is, the scale of the $\alpha$ associated with each high-energy splitting vertex. In earlier work [7], we asserted based on qualitative physical reasoning that this coupling should be taken to be $\alpha\left(Q_{\perp}\right)$, where $Q_{\perp}$ is the characteristic transverse momentum scale of the LPM-modified splitting process. Our explicit next-toleading-order LPM results bear this out. For roughly-democratic splitting processes, we find that the logarithmic terms in our NLO results for single splitting are ${ }^{8}$

$$
\left[\frac{d \Gamma}{d x}\right]_{e \rightarrow \gamma e}=\left[\frac{d \Gamma}{d x}\right]_{e \rightarrow \gamma e}^{\mathrm{LO}}\left[1+\beta_{0} \alpha \ln \left(\frac{|E \Omega|^{1 / 2}}{\mu}\right)+\cdots\right],
$$

where $\mu$ is the renormalization scale for $\alpha(\mu), \beta_{0}$ is the first coefficient of the renormalization group $\beta$-function for $\alpha$, and $|\Omega|$ is a frequency that is of order the inverse formation time. To avoid poor convergence of the perturbative expansion due to large logarithms, one should therefore choose $\mu \sim|E \Omega|^{1 / 2}$ above. As we review in section 2 , this is indeed equivalent (for roughly-democratic splittings) to $\mu \sim Q_{\perp}$.

The other feature regards our result for the effect of overlapping formation times on the real double splitting process $e \rightarrow \gamma e \rightarrow e \bar{e} e$. Though our analytic result is complicated for the general case, there is a simple leading-log formula in the limit that the intermediate photon is soft. That formula can be derived with a relatively simple analysis based on (i) the leading-order LPM formula for pair production $\gamma \rightarrow e \bar{e}$ combined with (ii)

\footnotetext{
${ }^{8}$ Specifically, see eq. (4.39) later in this paper, specializing here to the roughly-democratic case where neither $x_{e}$ nor $1-x_{e}$ are small. We have not drawn from our results any conclusions about the best renormalization scale in limiting cases such as $1-x_{e} \ll 1$, because then the logarithmic corrections due to choice of renormalization scale are overwhelmed by other, power-law corrections which dominate. Those power-law corrections are the same parametric size as $(2.21)$.
} 


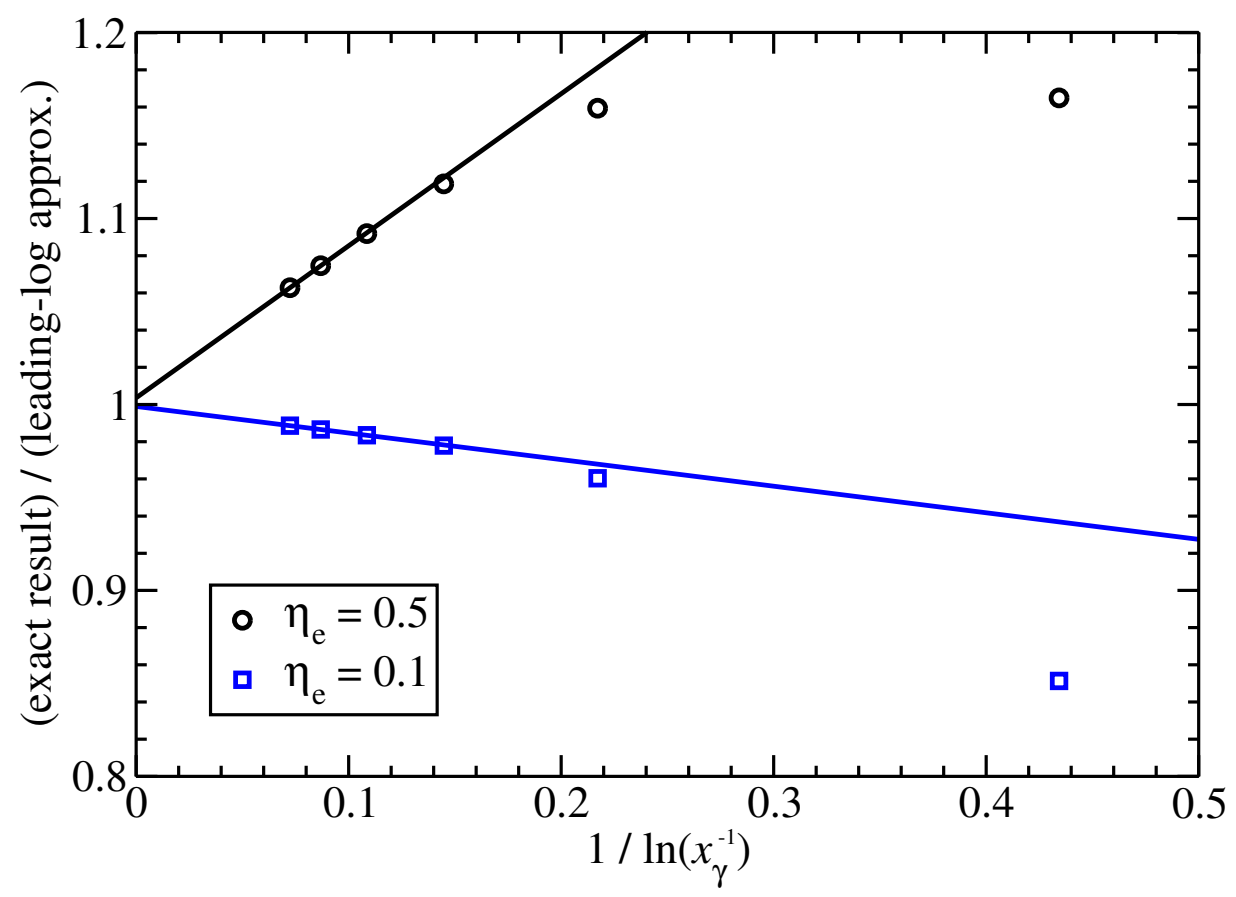

Figure 12. A plot demonstrating the success of the leading-log approximation (1.4) as $x_{\gamma} \rightarrow 0$. The vertical axis is the ratio of full numerical results to the leading-log approximation, and the horizontal axis is the inverse logarithm $1 / \ln \left(x_{\gamma}^{-1}\right)$. The data points come from numerical calculations of our general $e \rightarrow e \bar{e} e$ results, given in appendix A.2. The extrapolation to $x_{\gamma} \rightarrow 0$ is taken by a straight-line fit to the leftmost two data points of each set. Extrapolations are shown for two different values of $\mathfrak{y}_{e}$.

Dokshitzer-Gribov-Lipatov-Altarelli-Parisi (DGLAP) evolution of parton distributions to get the probability of seeing the photon $\gamma$ inside the initial electron $e$. We explain this analysis in section 2 , which yields the formula

$$
\left[\Delta \frac{d \Gamma}{d x_{\gamma} d \mathfrak{y}_{e}}\right]_{e \rightarrow e \bar{e} e} \approx \frac{N_{\mathrm{f}} \alpha^{2}\left[\mathfrak{y}_{e}^{2}+\left(1-\mathfrak{y}_{e}\right)^{2}\right]}{2 \pi^{2}\left[\mathfrak{y}_{e}\left(1-\mathfrak{y}_{e}\right)\right]^{1 / 2} x_{\gamma}^{3 / 2}} \ln \left(\frac{1}{x_{\gamma}\left[\mathfrak{y}_{e}\left(1-\mathfrak{y}_{e}\right)\right]^{1 / 2}}\right) \sqrt{\frac{\hat{q}}{E}} \quad\left(x_{\gamma} \ll 1\right),
$$

where $x_{\gamma}$ is the longitudinal momentum fraction of the intermediate photon relative to the initial electron in the bremsstrahlung $e \rightarrow \gamma e$, and $\mathfrak{y}_{e}$ is the longitudinal momentum fraction of the final electron relative to the photon in the subsequent pair production $\gamma \rightarrow e \bar{e}$. The $\approx \operatorname{sign}$ in (1.4) is our notation for indicating that this formula is only valid at leading-log order. The $\Delta$ in front of $d \Gamma / d x_{\gamma} d \mathfrak{n}_{e}$ is our notation [8] to denote the effect of overlapping formation times on rates for double splitting. That is, $\Delta\left[d \Gamma / d x_{\gamma} d \mathfrak{n}_{e}\right]$ is the difference between (i) $d \Gamma / d x_{\gamma} d \mathfrak{y}_{e}$ and (ii) what one would get by always treating the two consecutive medium-induced splittings $e \rightarrow \gamma e$ and $\gamma \rightarrow e \bar{e}$ as quantum-mechanically independent. Figure 12 verifies the leading-log approximation by showing that the ratio of our full numerical results for $e \rightarrow e \bar{e} e$ divided by the approximation (1.4) extrapolates to 1 as $x_{\gamma} \rightarrow 0$. The convergence is slow because logarithms grow slowly. 
As we will discuss in section 2, there are two interesting qualitative differences between QED $e \rightarrow e \bar{e} e$ and QCD $g \rightarrow g g g$ : (i) the $x_{\gamma} \ll 1$ behavior (1.4) will not give rise to any infrared (IR) divergences in energy-loss calculations, and so needs no IR cancellation between real and virtual diagrams, and (ii) the in-medium collinear logarithmic enhancement factor in (1.4) does not cancel between real double-splitting diagrams as it did for $g \rightarrow g g g$ [8] via a Gunion-Bertsch cancellation. ${ }^{9}$ This reflects a difference between soft pair production and soft bremsstrahlung.

Even though QED is qualitatively different from QCD in these respects, it nonetheless provides a good training ground for working out calculational methods for overlap effects in QCD. And overlap effects in QED are interesting in their own right, even if so far only treated here in the large- $N_{\mathrm{f}}$ limit.

\subsection{Outline}

We have tried to organize this paper so that the main text gives an introduction and overview of the techniques we use while the fine details of the calculation are left to an extensive set of appendices. In section 2, we summarize qualitative differences between QCD and (large- $N_{\mathrm{f}}$ ) QED regarding the LPM effect in both single-splitting and overlapping double-splitting processes. A quantitative review of single-splitting formulas is left to appendix A.1, and appendix C contains a translation of modern notation using $\hat{q}$ to the QED results originally presented by Migdal [3]. Section 3 introduces the elements needed for organizing calculations of overlapping formation-time effects in terms of light-cone perturbation theory (LCPT), with the full list of details left to appendix D. The use of those rules to calculate overlap effects in the real double-splitting process $e \rightarrow e \bar{e} e$ of figure 7 is left to appendix E, where much of the calculation is adapted from our previous work on $g \rightarrow g g g$ in QCD. In section 4, we turn to the virtual corrections of figures 8 and 9 . We first discuss the techniques needed, which we call back- and front-end transformations, to easily relate almost all virtual diagrams to non-virtual diagrams. We then turn to the one remaining virtual diagram (the boxed diagram of figure $8 \mathrm{k}$ ) and outline its computation and renormalization but leave details for appendices F and G. ${ }^{10}$ Finally, we end with a brief conclusion in section 5. A complete summary of the next-to-leading order LPM rate formulas derived in this paper is given in appendix A.

\subsection{Reference acronyms}

When discussing detailed formulas in appendices and sometime footnotes, we will often need to refer to particular sections or equations of our earlier work [7-10]. To streamline such references, we will often refer to our earlier work in such cases by the author acronyms and numbers AI1 [7], ACI2 [8], ACI3 [9], and ACI4 [10]. So, for instance, "ACI3 (3.4)" will be shorthand for eq. (3.4) of ref. [9], and "AI1 section II.A" will be shorthand for section II.A of ref. [7].

\footnotetext{
${ }^{9}$ Specifically, see appendix B of ref. [8].

${ }^{10}$ If, like us, you think it's really fun to figure out how to do a new type of dimensionally-regularized integral, then appendix $\mathrm{G}$ is the appendix for you.
} 


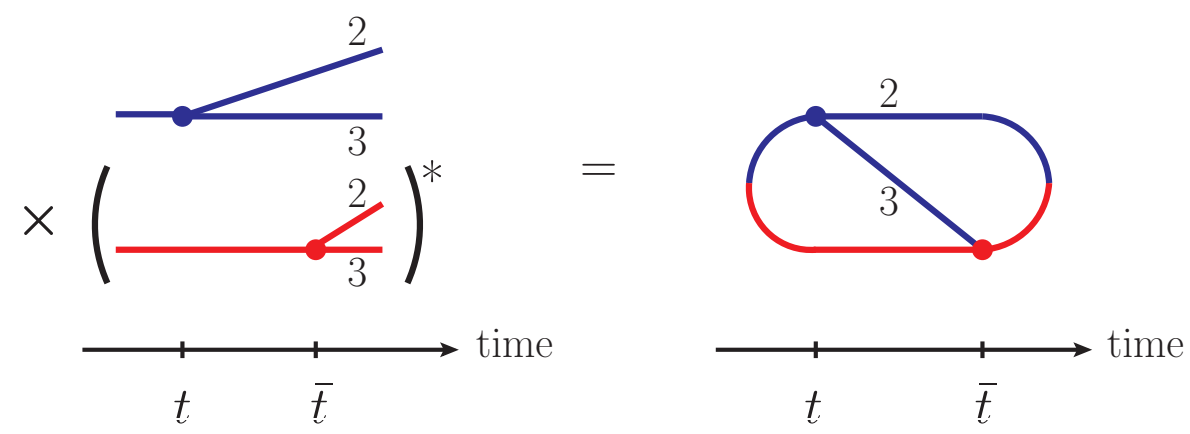

Figure 13. Schematic (time-ordered) interference diagram for a leading-order single-splitting process $E \rightarrow x_{2} E$ and $x_{3} E$, where here the lines can be any particle type.

\section{Qualitative differences between QCD and QED}

\subsection{Single splitting}

The crucial difference to keep in mind between the LPM effect in QCD and QED is that the photon is neutral but the gluon has color charge. In terms of parametric behavior, this makes a huge difference in how LPM suppression behaves when a bremsstrahlung photon or gluon is soft. The LPM effect relies on the near-collinearity of high-energy splitting processes, and the formation time $l_{\text {form }}$ (and so the amount of LPM rate suppression) is smaller when the splitting is less collinear. Since it is easier to deflect a low-momentum particle than a high-momentum particle, the collinearity of QCD gluon bremsstrahlung is controlled by how soft the bremsstrahlung gluon is: high-energy gluon bremsstrahlung is less LPM-suppressed the softer the bremsstrahlung gluon is. A soft photon, however, does not scatter in first approximation, and so the collinearity of QED photon bremsstrahlung is insensitive to this. But a softer photon means a longer-wavelength photon, which means a photon with less resolving power, which is why the QED case has the opposite behavior: high-energy photon bremsstrahlung is more LPM-suppressed the softer the photon is.

\subsubsection{Formation times}

A little more concretely, let's review, in a general way, some standard parametric estimates of formation times. Consider a single splitting process of a high-energy particle with energy $E$ into daughters of energy $E_{2}=x_{2} E$ and $E_{3}=x_{3} E$ and ask for the formation time. That's the time scale $|t-\bar{t}|$ over which it's possible for splitting in the amplitude at time $t$ to interfere with splitting in the conjugate amplitude at time $\bar{t}$, as depicted by the interference diagram in figure 13 . That time scale $t_{\text {form }}$ is determined by the difference $\delta E$ of the energies of (i) the two-particle state $|2,3\rangle$ after splitting in the amplitude and (ii) the one-particle state before splitting in the conjugate amplitude:

$$
\delta E=\left(E_{\boldsymbol{p}_{2}}+E_{\boldsymbol{p}_{3}}\right)-E_{\boldsymbol{p}_{1}} \simeq-\frac{\boldsymbol{p}_{1}^{2}+m_{1}^{2}}{2 E}+\frac{\boldsymbol{p}_{2}^{2}+m_{2}^{2}}{2 E_{2}}+\frac{\boldsymbol{p}_{3}^{2}+m_{2}^{2}}{2 E_{2}},
$$

where $\boldsymbol{p}_{i}$ are the transverse momenta, which we take to be small compared the energies $E_{i}$. In this paper, we will assume throughout that energies are high enough that we can ignore 
the effective masses $m_{i}$ of the high-energy particles in the medium. One can then make a rough parametric estimate $t \sim 1 /|\delta E|$ of the formation time by using the definition of $\hat{q}$ to take $\boldsymbol{p}_{i}^{2} \sim \hat{q}_{i} t$ above, where $\hat{q}_{i}$ is the $\hat{q}$ appropriate for particles of type $i$. Then, from (2.1),

$$
t \sim \frac{1}{|\delta E|} \sim\left[-\frac{\hat{q}_{1} t}{2 E}+\frac{\hat{q}_{2} t}{2 x_{2} E}+\frac{\hat{q}_{3} t}{2 x_{3} E}\right]^{-1}
$$

and so

$$
t_{\text {form }} \sim \sqrt{\frac{E}{-\hat{q}_{1}+\left(\hat{q}_{2} / x_{2}\right)+\left(\hat{q}_{3} / x_{3}\right)}} .
$$

For photon bremsstrahlung $e \rightarrow \gamma e$, we have $\hat{q}_{\gamma}=0$, and (2.3) gives

$$
t_{\text {form }}^{e \rightarrow \gamma e} \sim \sqrt{\frac{E}{-\hat{q}+\left(\hat{q} / x_{e}\right)}}=\sqrt{\frac{x_{e} E}{\left(1-x_{e}\right) \hat{q}}}=\sqrt{\frac{\left(1-x_{\gamma}\right) E}{x_{\gamma} \hat{q}}},
$$

where $\hat{q} \equiv \hat{q}_{e}$. Above, $x_{e}$ and $x_{\gamma}$ (which in this case is $x_{\gamma}=1-x_{e}$ ) are respectively the longitudinal momentum fractions of the electron and photon daughters of the splitting. In contrast, for gluon bremsstrahlung $q \rightarrow q g$ or $g \rightarrow g g$, we have $\hat{q}_{q} \sim \hat{q}_{g}$ and

$$
t_{\text {form }}^{X \rightarrow g X} \sim \sqrt{\frac{E}{\left.-\hat{q}_{X}+\left(\hat{q}_{g} / x_{g}\right)+\left(\hat{q}_{X} /\left(1-x_{g}\right)\right)\right)}} \sim \sqrt{\frac{x_{g}\left(1-x_{g}\right) E}{\hat{q}_{\mathrm{QCD}}}} .
$$

The parametric estimates (2.4) and (2.5) show that the photon formation time becomes long (more LPM suppression) as $x_{\gamma} \rightarrow 0$ whereas the gluon formation time becomes short (less LPM suppression) as $x_{g} \rightarrow 0$.

We've used the very general formula (2.3) to derive standard parametric estimates of LPM formation times because it is then easy in the same breath to obtain the standard parametric result for QED pair production $\gamma \rightarrow e \bar{e}$, which is qualitatively different from photon bremsstrahlung. (2.3) gives

$$
t_{\text {form }}^{\gamma \rightarrow e \bar{e}} \sim \sqrt{\frac{E}{\left(\hat{q} /\left(1-x_{e}\right)\right)+\left(\hat{q} / x_{e}\right)}}=\sqrt{\frac{x_{e}\left(1-x_{e}\right) E}{\hat{q}}} .
$$

The QCD pair production formation time $(g \rightarrow q \bar{q})$ is parametrically similar to this, as well as to the QCD bremsstrahlung formation time (2.5).

\subsubsection{Splitting rates}

Below, we will need the differential rates $d \Gamma / d x$ for single splitting. For nearly-democratic splittings, the relation given by (1.1) is that $\Gamma \sim \alpha / t_{\text {form }}$. But we will also be interested in non-democratic splittings (one daughter soft) and will want to discuss the differential rate $d \Gamma / d x$, where $x$ is one of the daughter momentum fractions. The differential rate is sensitive to the DGLAP splitting function for the process:

$$
\frac{d \Gamma^{1 \rightarrow 23}}{d x} \sim \frac{\alpha P_{1 \rightarrow 23}(x)}{t_{\text {form }}^{1 \rightarrow 23}},
$$


which gives

$$
\left[\frac{d \Gamma}{d x}\right]_{\text {brem }} \sim \frac{\alpha}{x_{\gamma, g} t_{\text {form }}}
$$

for bremsstrahlung $\left(e \rightarrow \gamma e\right.$ or $X \rightarrow g X$, with $x_{g}$ being the softest gluon in the case $g \rightarrow g g)$ and

$$
\left[\frac{d \Gamma}{d x}\right]_{\text {pair }} \sim \frac{N_{\mathrm{f}} \alpha}{t_{\mathrm{form}}}
$$

for pair production $(\gamma \rightarrow e \bar{e}$ or $g \rightarrow q \bar{q})$.

For a review of precise, quantitative formulas for single splitting rates (in the high energy limit where the $\hat{q}$ approximation can be used), see appendix A.1. Appendix C discusses exactly how, in the QED case, these formulas match up to the original results presented by Migdal [3] in the case where the medium is an atomic gas.

\subsubsection{An aside on nearly-democratic splitting}

We mention in passing that QED and QCD are parametrically similar in the case of nearlydemocratic splitting (neither daughter soft compared to the parent). All the formation times discussed above then have the same order of magnitude:

$$
t_{\text {form }} \sim \sqrt{\frac{E}{\hat{q}}} \quad \text { (nearly-democratic splitting). }
$$

(The splitting rates are then also all parametrically the same except for factors of $N_{\mathrm{f}}$.) The amount of transverse momentum $Q_{\perp}$ transferred from the medium in one formation time is of order $Q_{\perp}^{2} \sim \hat{q} t_{\text {form }}$, which can be written in a number of equivalent ways:

$$
Q_{\perp} \sim\left(\hat{q} t_{\text {form }}\right)^{1 / 2} \sim(\hat{q} E)^{1 / 4} \sim\left(\frac{E}{t_{\text {form }}}\right)^{1 / 2} \quad \text { (nearly-democratic splitting). }
$$

We used the last form to identify $\mu \sim|E \Omega|^{1 / 2}$ with $Q_{\perp}$ in the discussion of renormalization scale in section 1.4.

One could of course also write down case-by-case parametric estimates of $Q_{\perp}$ for nondemocratic splittings, but we do not have need of them.

\subsection{Overlapping double splitting}

In ref. [8] (ACI2), ${ }^{11}$ we discussed how to parametrically estimate the size (though not the sign) of QCD overlapping formation-time effects for real double splitting $g \rightarrow g g g$ by using formation times to estimate rates. Here we will first review that QCD estimate and then see what changes when we switch to (large- $\left.N_{\mathrm{f}}\right)$ QED.

\subsubsection{Review of QCD estimate for $g \rightarrow g g g$}

Let $y E$ and $x E$ be the energies of the softest and next-softest of the three final-state gluons, so that $y \lesssim x \lesssim 1-x-y$. Since this is $\mathrm{QCD}$, the formation time $t_{\text {form, } y}$ for radiating the

\footnotetext{
${ }^{11}$ Specifically ACI2 section I.D.
} 
$y$ gluon will be shorter than the formation time $t_{\text {form }, x}$ for radiating the $x$ gluon. The probability that a $y$ emission happens to take place during the $x$ emission is then just $t_{\text {form, } \mathrm{x}}$ times the rate of $y$ emission. Overall, that means that the joint differential rate for overlapping $x$ and $y$ emissions is

$$
\left[\frac{d \Gamma}{d x d y}\right]_{\text {overlap }} \sim \frac{d \Gamma_{x}}{d x} \times \frac{d \Gamma_{y}}{d y} t_{\text {form }, x}
$$

Using (2.8), that's

$$
\left[\frac{d \Gamma}{d x d y}\right]_{\text {overlap }} \sim \frac{\alpha_{\mathrm{s}}^{2}}{x y t_{\text {form }, y}}
$$

and thence, from (2.5),

$$
\left[\frac{d \Gamma}{d x d y}\right]_{\text {overlap }} \sim \frac{\alpha_{\mathrm{s}}^{2}}{x y^{3 / 2}} \sqrt{\frac{\hat{q}}{E}} .
$$

The fact that two emissions overlap does not a priori mean they will influence each other, and so the qualitative argument for (2.14) only provides an estimate for how large overlap effects might be. However, detailed calculations [8] of overlap effects for $g \rightarrow g g g$ confirm it.

Note that if one multiples $(2.14)$ by the energy $(x+y) E$ lost by the leading parton and integrates over $x$ and $y$, one would find a power-law divergent contribution from overlap effects to energy loss. No such power-law divergence appears in the soft-gluon bremsstrahlung calculations of refs. [4-6], but those calculations inextricably combine the effects of soft virtual emission with those of soft real emission, for which there are cancellations in QCD. Here we have only estimated the size of soft real emission alone.

\subsubsection{QED estimate for $e \rightarrow e \bar{e} e$}

For large- $N_{\mathrm{f}}$ QED we've already identified that the dominant overlap correction comes from the $e \rightarrow e \bar{e} e$ process of figure 6 . Let $x_{e} E$ and $y_{e} E$ be the energies of the two final-state electrons. As shown in the figure, our convention throughout this paper will be that $x_{e}$ is the daughter whose electron line is connected to the initial-state electron, and $y_{e}$ is the electron in the $e \bar{e}$ pair produced by the intermediate photon. This is a distinction made possible by the large- $N_{\mathrm{f}}$ limit, in which the chance that the electron in the $\gamma \rightarrow e \bar{e}$ pair has the same flavor as the initial electron (which would allow interference terms involving exchange of the two electrons) is $1 / N_{\mathrm{f}}$ suppressed. In this section, we will focus on the case $x_{e} \sim 1$, which includes the case $x_{\gamma} \rightarrow 0$ but not $x_{e} \rightarrow 0$. (Our later explicit calculations of diagrams make no such assumption.)

The formation time for the initial bremsstrahlung $e \rightarrow \gamma e$ can be taken from (2.4):

$$
t_{\text {form }, x} \sim \sqrt{\frac{E}{x_{\gamma} \hat{q}}} \quad\left(x_{e} \sim 1\right) .
$$

To get the formation time for the subsequent pair production process $\gamma \rightarrow e \bar{e}$, we have to be careful applying the single splitting estimate (2.6) because we are considering the case where the photon is already soft. The $E$ in (2.6) is the photon energy, which we are now 
calling $x_{\gamma} E$. The $x_{e}$ in (2.6) is the longitudinal momentum fraction of the pair's electron relative to the photon, which in the application here (see figure 6) is

$$
\mathfrak{y}_{e} \equiv \frac{y_{e} E}{x_{\gamma} E}=\frac{y_{e}}{1-x_{e}} .
$$

With these substitutions, (2.6) gives

$$
t_{\text {form }, y} \sim \sqrt{\frac{\mathfrak{y}_{e}\left(1-\mathfrak{y}_{e}\right) x_{\gamma} E}{\hat{q}}}
$$

in the present context. As in the QCD estimate, this is $\lesssim t_{\text {form }, x}$, and so we can estimate the probability of overlap the same way, using (2.12), which can also be written

$$
\left[\frac{d \Gamma}{d x d \mathfrak{y}_{e}}\right]_{\text {overlap }} \sim \frac{d \Gamma_{x}}{d x} \times \frac{d \Gamma_{y}}{d \mathfrak{y}_{e}} t_{\text {form }, x}
$$

Here we need to make sure to use the pair-production formula (2.9) to get $d \Gamma / d \mathfrak{y}_{e} \sim$ $N_{\mathrm{f}} \alpha / t_{\text {form, } y}$. So, using (2.17) above,

$$
\left[\frac{d \Gamma}{d x_{e} d \mathfrak{y}_{e}}\right]_{\text {overlap }} \sim \frac{N_{\mathrm{f}} \alpha^{2}}{x_{\gamma} t_{\text {form }, y}} \sim \frac{N_{\mathrm{f}} \alpha^{2}}{x_{\gamma}^{3 / 2}\left[\min \left(\mathfrak{y}_{e}, 1-\mathfrak{y}_{e}\right)\right]^{1 / 2}} \sqrt{\frac{\hat{q}}{E}} \quad\left(x_{e} \sim 1\right) .
$$

Equivalently, using (2.16),

$$
\left[\frac{d \Gamma}{d x_{e} d y_{e}}\right]_{\text {overlap }} \sim \frac{N_{\mathrm{f}} \alpha^{2}}{x_{\gamma}^{3 / 2}\left[\min \left(y_{e}, x_{\gamma}-y_{e}\right)\right]^{1 / 2}} \sqrt{\frac{\hat{q}}{E}} .
$$

Unlike the QCD case, the integral over $y_{e}$ does not diverge:

$$
\left[\frac{d \Gamma}{d x_{e}}\right]_{\text {overlap }}^{e \rightarrow e \bar{e} e} \sim \frac{N_{\mathrm{f}} \alpha^{2}}{x_{\gamma}^{3 / 2}} \int_{0}^{1} \frac{d \mathfrak{y}_{e}}{\left[\min \left(\mathfrak{y}_{e}, 1-\mathfrak{y}_{e}\right)\right]^{1 / 2}} \sim \frac{N_{\mathrm{f}} \alpha^{2}}{x_{\gamma}^{3 / 2}} \quad\left(x_{e} \sim 1\right) .
$$

Furthermore, were we to use this formula to calculate the overlap effects on energy loss, we would get finite results, even without accounting (as one still should) for virtual corrections. QED has much better infrared behavior than QCD for these calculation because the LPM suppression of photon radiation increases as the photon becomes softer.

\subsubsection{Leading-log formula for $e \rightarrow e \bar{e} e$}

Before moving on to the details of our complete calculation of overlap effects in the general case, we first briefly discuss a relatively simple argument for the leading-log formula (1.4) for $x_{\gamma} \ll 1$. As mentioned earlier, the existence of such a logarithm is another difference between $e \rightarrow e \bar{e} e$ and $g \rightarrow g g g$.

Consider the $e \rightarrow e \bar{e} e$ process of figure 6 and re-interpret it as the Feynman diagram shown in figure 14. In the latter figure, we have not explicitly drawn the (still overlapping) ovals corresponding to formation times. Instead, the box here denotes the leading-order pair-production process $\gamma \rightarrow e \bar{e}$ via interaction with the medium, and we will consider that 


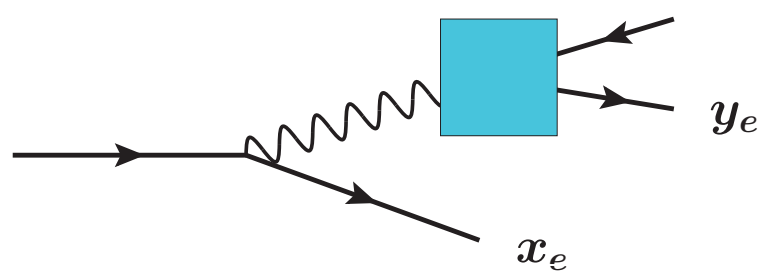

Figure 14. The double splitting process $e \rightarrow \gamma e \rightarrow e \bar{e} e$ viewed in an approximation (relevant to overlap effects in the limit $x_{\gamma} \ll 1$ ) treating it as fundamentally pair production $\gamma \rightarrow e \bar{e}$ with the $\gamma$ interpreted as a parton inside the original electron, described by DGLAP evolution.

photon $\gamma$ as a parton contained inside the initial electron. In a related context, ref. [8] (ACI2) argues that the corresponding parton-model-like (i.e. DGLAP) approximation to such a process has the generic form ${ }^{12}$

$$
\frac{d \Gamma}{d x d y} \approx \frac{\alpha}{2 \pi} P(1-x) \ln \left(\frac{Q^{2}}{Q_{0}^{2}}\right) \frac{d \Gamma}{d y} \approx \frac{\alpha}{2 \pi} P(1-x) \ln \left(\frac{t_{\text {form }, x}}{t_{\text {form }, y}}\right) \frac{d \Gamma}{d y},
$$

where $d \Gamma / d y$ is the differential rate for the fundamental parton-level process (represented here by the box in figure 14) and here the rest of (2.22) represents the probability of finding the photon inside the initial electron. The analysis of that probability is different from what it would be in vacuum because scattering from the medium cuts off collinear logarithms. [See ref. [8] for the argument that the DGLAP logarithm $\ln \left(Q^{2} / Q_{0}^{2}\right)$ of the virtuality ratio translates to the logarithm $\ln \left(t_{\text {form }, x} / t_{\text {form, } y}\right)$ of the formation-time ratio.] The translation of (2.22) to figure 14 is then

$$
\frac{d \Gamma}{d x_{e} d y_{e}} \approx \frac{\alpha}{2 \pi} P_{e \rightarrow e}\left(x_{e}\right) \ln \left(\frac{t_{\text {form }, x}}{t_{\text {form }, y}}\right)\left[\frac{d \Gamma}{d y_{e}}\right]_{\gamma \rightarrow e \bar{e}}^{\mathrm{LO}}
$$

or equivalently

$$
\frac{d \Gamma}{d x_{\gamma} d \mathfrak{y}_{e}} \approx \frac{\alpha}{2 \pi} P_{e \rightarrow \gamma}\left(x_{\gamma}\right) \ln \left(\frac{t_{\text {form }, x}}{t_{\text {form }, y}}\right)\left[\frac{d \Gamma}{d \mathfrak{y}_{e}}\right]_{\gamma \rightarrow e \bar{e}}^{\mathrm{LO}} .
$$

The parametric estimate of $d \Gamma / d \mathfrak{y}_{e}$ was given earlier as $\sim N_{\mathrm{f}} \alpha / t_{\text {form, } y} \sim N_{\mathrm{f}} \alpha \sqrt{\hat{q} / \mathfrak{y}_{e}\left(1-\mathfrak{y}_{e}\right) E}$, but here we want the exact leading-order result. That's reviewed in appendix A.1 and (using the same translations as in our parametric analysis) is

$$
\left[\frac{d \Gamma}{d \mathfrak{y}_{e}}\right]_{\gamma \rightarrow e \bar{e}}^{\mathrm{LO}}=\frac{N_{\mathrm{f}} \alpha}{\pi} P_{\gamma \rightarrow e}\left(\mathfrak{y}_{e}\right) \operatorname{Re}\left(i \Omega_{0}^{\gamma \rightarrow e \bar{e}}\right)
$$

here, with

$$
\Omega_{0}^{\gamma \rightarrow e \bar{e}}=\sqrt{\frac{-i \hat{q}}{2 \mathfrak{y}_{e}\left(1-\mathfrak{y}_{e}\right) E_{\gamma}}}=\sqrt{\frac{-i \hat{q}}{2 \mathfrak{y}_{e}\left(1-\mathfrak{y}_{e}\right) x_{\gamma} E}} .
$$

Combining (2.24)-(2.26) with the formation times (2.15) and (2.17), using the explicit formulas for the DGLAP splitting functions [see (A.6) and (A.10)], and taking the limit $x_{\gamma} \ll 1$ (and hence $x_{e} \rightarrow 1$ ) yields the leading-log approximation (1.4) quoted earlier and verified from our full calculation of overlap effects.

\footnotetext{
${ }^{12}$ See specifically ACI2 eqs. (B5-B6) in ACI2 appendix B.1 [8].
} 


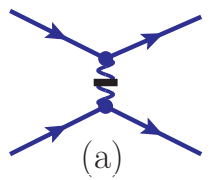

(a)

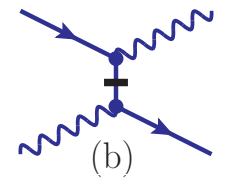

(b)

Figure 15. (a) Depiction of the interactions that arise in QED after integrating out longitudinalpolarized photons in light-cone gauge. (b) Similar interaction arising in LCPT from integrating out unphysical fermion states.

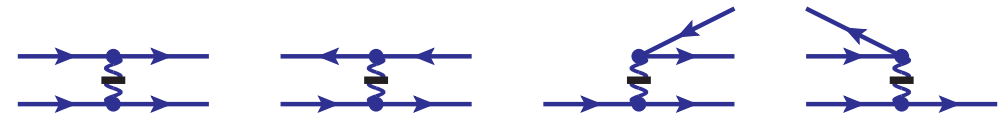

Figure 16. Various light-cone time ordered versions of figure 15a, appropriate for Hamiltonian formalism. Light-cone time runs from left to right.

\section{Light cone perturbation theory for overlapping LPM}

\subsection{Diagrammatic rules and their translation}

Our earlier work did not include the effects of longitudinally-polarized gauge bosons in intermediate states. One may retain a description solely in terms of transverse polarizations by first integrating out the longitudinal polarizations in light-cone gauge, as in Light Cone Perturbation Theory. This gives rise to 4-fermion interactions such as shown in figure 15a, which are instantaneous in light-cone time $x^{+}$, local in transverse position $\boldsymbol{x}^{\perp}$, and nonlocal in $x^{-}$. In Hamiltonian formalism (as in LCPT), the legs can be incoming or outgoing, such as shown in figure 16 for figure 15a. In LCPT, a similar 4-particle interaction arises from fermion exchange, corresponding to figure 15b. Loosely speaking, this interaction term accounts for the difference between using (i) the actual off-shell exchanged fermion and (ii) treating that fermion as though it had on-shell polarization $u_{p}$ or $v_{p}$. An important feature of LCPT is that figure 15 for QED (and similar 4-field diagrams for QCD) is all that is needed to account for the effects of longitudinal gauge bosons or off-shell fermion polarizations: there are no $n$-field interaction terms in the light-cone Hamiltonian with $n>4$.

Figures 17 and 18 show two examples of standard LCPT rules for vertices (shown inside the boxes) and examples of their translation to corresponding vertices of interference diagrams in the reduced-particle description used in our earlier work [7, 10], such as depicted by figure 11b. Other basic vertices are covered in appendix D. In the language of figure 11a, the interference diagram elements, shown outside the boxes in figures 17 and 18, represent vertices for $2 \rightarrow 3$ and $2 \rightarrow 4$ particle transitions respectively. In the reduced-particle description of figure $11 \mathrm{~b}$, these become effectively $0 \rightarrow 1$ and $0 \rightarrow 2$ transitions, with the states described by one transverse momentum or position variable $(\boldsymbol{P}$ or $\boldsymbol{B})$ per effective particle.

The first expression for $\langle\boldsymbol{P}|-i \delta H|\rangle$ in figure 17 shows the general rule for converting ordinary matrix elements (the boxed formula) into the conventions of AI1 [7] for effectively $0 \rightarrow 1$ particle transitions. The only change here to the $\delta H$ matrix element is that the normalization conventions of AI1 [7] include a factor of $\left|2 E_{n}\right|^{-1 / 2}$ for each individual particle state. Our convention is that $E_{n}=x_{n} E$, where $E$ is the energy of the original high-energy 


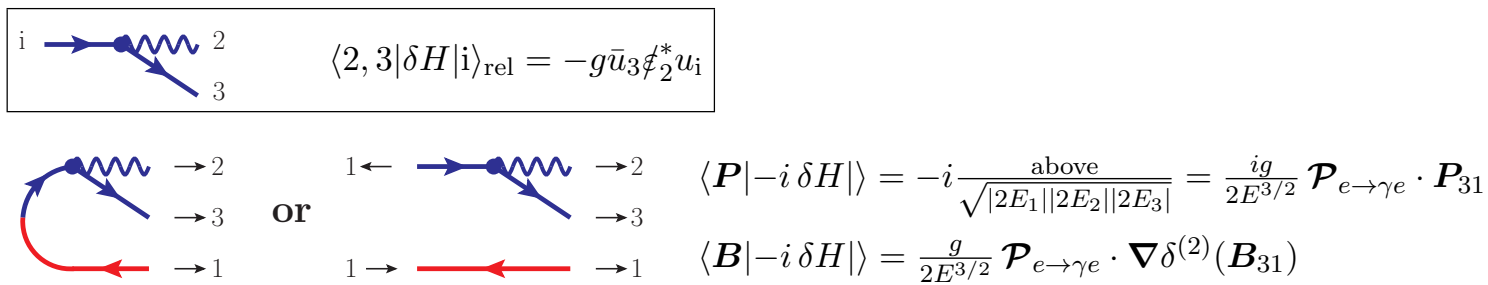

Figure 17. The box shows the Hamiltonian matrix element for $e \rightarrow \gamma e$, as in LCPT, normalized with relativistic normalization as in [14]. The next line shows the corresponding formula, in the normalization and conventions of AI1 [7], for an initial splitting $e \rightarrow \gamma e$ in the amplitude (as opposed to conjugate amplitude), like the left-most (earliest time) vertex in figure $11 \mathrm{~b}$ or in figures $7(\mathrm{a}-\mathrm{c})$ and 8 (h-k). As written, the latter formulas only apply to $2 \rightarrow 3$ particle transitions in the language of figure 11a, which is equivalent to effectively $0 \rightarrow 1$ particle transitions in the language of figure $11 \mathrm{~b}$. Note that these formulas show the matrix element of $-i \delta H$ rather than $\delta H$, according to the convention of AI1 [7]. [The origin of the $-i$ factor is the $-i$ in the evolution operator $e^{-i H t}$ for amplitudes.]

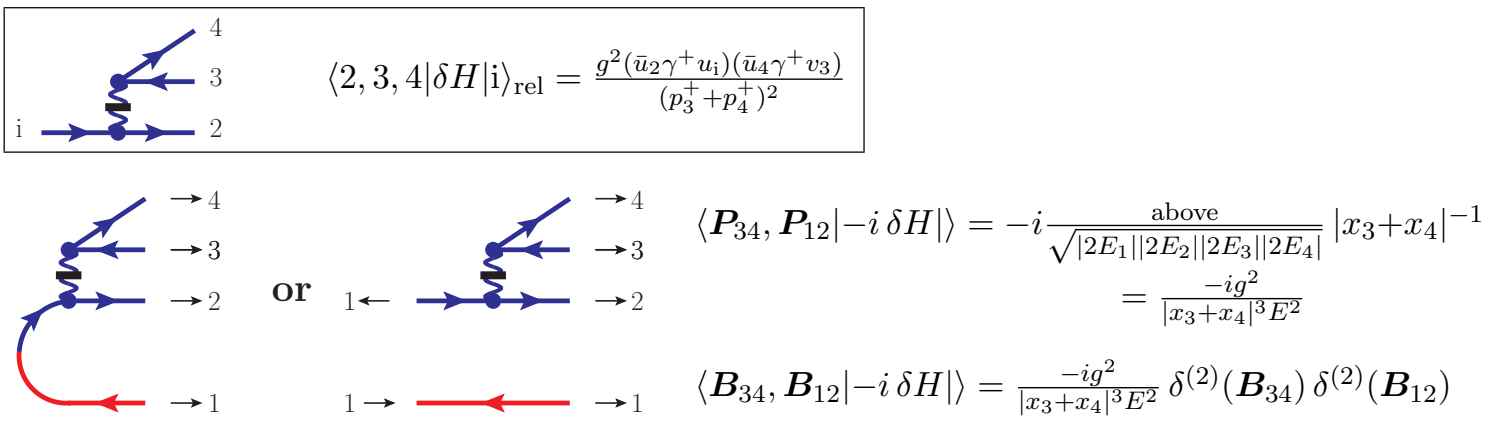

Figure 18. Like figure 17 but for an example of an "instantaneous" LCPT interaction, shown in the box. Here, in the language of figure 11, the formulas outside of the box give an example of a $2 \rightarrow 4$ (effectively $0 \rightarrow 2$ ) transition. Chirality is conserved following each fermion line through the vertex.

parent at the very start of the processes depicted in figures $7-9$, and where $x_{n}$ is the longitudinal momentum fraction of particle $n$ with the convention that $x_{n}$ is negative for particles in the conjugate amplitude, so that $\sum_{n} x_{n}$ is always zero.

The final expression for $\langle\boldsymbol{P}|-i \delta H|\rangle$ in figure 17 shows what you get if you express $\bar{u}_{3} \ddagger_{2}^{*} u_{\mathrm{i}}$ in the same notation used in our previous work [7]. Here

$$
\boldsymbol{P}_{i j} \equiv x_{j} \boldsymbol{p}_{i}-x_{i} \boldsymbol{p}_{j}
$$

where the $\boldsymbol{p}$ 's represent transverse momenta. For 3-particle (effectively 1-particle) states, the $\boldsymbol{P}_{i j}$ are related by momentum conservation: ${ }^{13}$

$$
\boldsymbol{P}_{12}=\boldsymbol{P}_{23}=\boldsymbol{P}_{31}
$$

\footnotetext{
${ }^{13}$ For a discussion of (3.1), (3.2) and (3.4) in the context of our application and notation here, see specifically AI1 sections II.E and III [7].
} 
The $\mathcal{P}$ are related to the square roots of helicity-dependent DGLAP splitting functions. Here,

$$
\mathcal{P}_{\mathrm{i} \rightarrow 2,3}\left(x_{\mathrm{i}} \rightarrow x_{2}, x_{3}\right)=\frac{\left(\boldsymbol{e}_{x} \pm i \boldsymbol{e}_{y}\right)}{\left|x_{\mathrm{i}} x_{2} x_{3}\right|} \sqrt{P_{\mathrm{i} \rightarrow 23}\left(x_{\mathrm{i}} \rightarrow x_{2}, x_{3}\right)}=\frac{\left(\boldsymbol{e}_{x} \pm i \boldsymbol{e}_{y}\right)}{\left|x_{\mathrm{i}} x_{2} x_{3}\right|} \sqrt{\left|x_{\mathrm{i}}\right| P_{\mathrm{i} \rightarrow 23}\left(1 \rightarrow z_{2}, z_{3}\right)},
$$

where $z_{n} \equiv x_{n} / x_{\mathrm{i}}$ are the momentum fractions of the daughters relative to their immediate parent, $P_{\mathrm{i} \rightarrow 23}\left(1 \rightarrow z_{2}, z_{3}\right)$ is the helicity-dependent DGLAP splitting function appropriate to the particle types and helicities of the initial and final particles in the splitting process, and the \pm in the circular basis vector $\boldsymbol{e}_{x} \pm i \boldsymbol{e}_{y}$ is chosen accordingly based on those helicities. ${ }^{14}$

The Fourier conjugate of $\boldsymbol{P}_{i j}$ is, in our notation,

$$
\boldsymbol{B}_{i j} \equiv \frac{\boldsymbol{b}_{i}-\boldsymbol{b}_{j}}{x_{i}+x_{j}}
$$

The $\langle\boldsymbol{B}|-i \delta H|\rangle$ formula in figure 17 is simply the Fourier transform of the $\langle\boldsymbol{P}|-i \delta H|\rangle$ formula.

Now turn to figure 18. The first expression for $\left\langle\boldsymbol{P}_{34}, \boldsymbol{P}_{12}|-i \delta H|\right\rangle$ there shows the general rule for converting ordinary matrix elements (the boxed formula) into the conventions of refs. [7, 10] (AI1,ACI3) for instantaneous, effectively $0 \rightarrow 2$ particle transitions. In addition to the $\left|2 E_{n}\right|^{-1 / 2}$ factors, there is an additional factor of $\left|x_{3}+x_{4}\right|^{-1}$ that is associated with the normalization of the state $\left|\boldsymbol{P}_{34}, \boldsymbol{P}_{12}\right\rangle$ that one obtains when reducing from a 4-particle description, of the state just after the interaction, to an effective 2-particle description. ${ }^{15}$ There is nothing special here about the pairing of the indices 1234 in this normalization factor; one could just as well have written

$$
\left\langle\boldsymbol{P}_{23}, \boldsymbol{P}_{41}|-i \delta H|\right\rangle=-i \frac{\text { above }}{\sqrt{\left|2 E_{1}\right|\left|2 E_{2}\right|\left|2 E_{3}\right|\left|2 E_{4}\right|}}\left|x_{1}+x_{4}\right|^{-1}
$$

in figure 18. (Note that $\left|x_{3}+x_{4}\right|^{-1}=\left|x_{1}+x_{2}\right|^{-1}$ and $\left|x_{1}+x_{4}\right|^{-1}=\left|x_{2}+x_{3}\right|^{-1}$ since $\sum_{n} x_{n}=$ 0$.$) The only advantage to choosing \left\langle\boldsymbol{P}_{34}, \boldsymbol{P}_{12}\right|$ in figure 18 is that then the $\left|x_{3}+x_{4}\right|^{-1}$ from the normalization factor there neatly combines with the $\left(x_{3}+x_{4}\right)^{-2}$ coming from the denominator $\left(p_{3}^{+}+p_{4}^{+}\right)^{-2}$ in the boxed LCPT rule. Finally, the $\left\langle\boldsymbol{B}_{34}, \boldsymbol{B}_{12}|-i \delta H|\right\rangle$ formula in figure 18 is just the Fourier transform of the $\left\langle\boldsymbol{P}_{34}, \boldsymbol{P}_{12}|-i \delta H|\right\rangle$ formula.

We need not state whether our convention for light-cone components is $v^{ \pm}=v^{0} \pm v^{3}$ or $v^{ \pm}=\left(v^{0} \pm v^{3}\right) / \sqrt{2}$ because it does not matter. The formulas for matrix elements, such as in figure 18 , give the same result either way.

From formulas such as figures 17 and 18 for basic interactions, many variations follow from substitutions and complex conjugation. As an example, the interference-diagram rule in figure 17 also gives the additional interference-diagram rules shown in figure 19. This procedure has the advantage of definiteness, but one may also formulate a more generic rule that covers all the different variations - see appendix D.

\footnotetext{
${ }^{14}$ See AI1 section IV.E and AI1 appendix C [7] for more details of our conventions.

${ }^{15}$ See specifically AI1 section IV.D and AI1 appendix B [7]. It is the same factor as in the bottom diagram of AI1 figure 16 .
} 

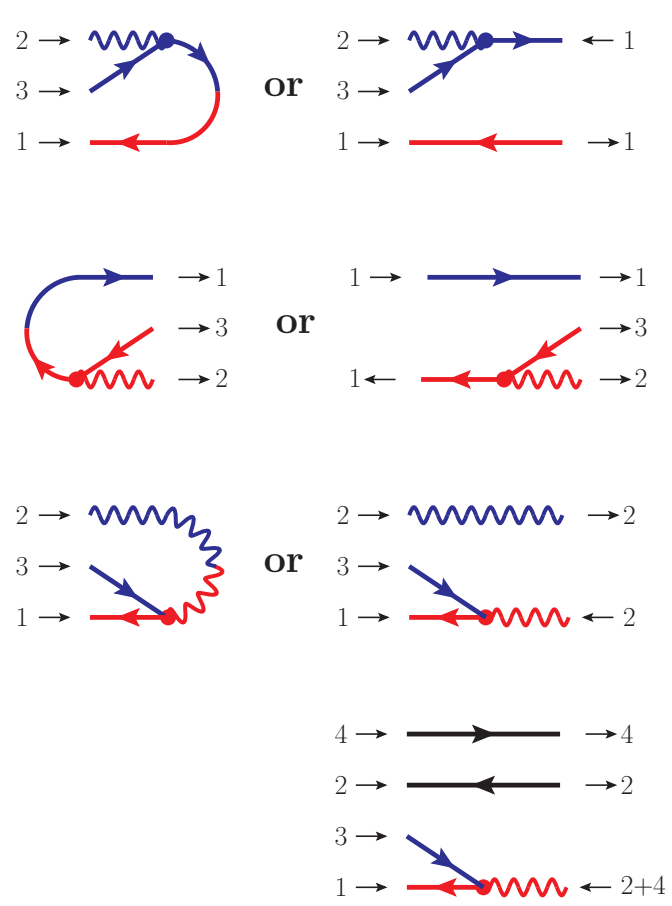

$$
\begin{aligned}
& \langle|-i \delta H| \boldsymbol{P}\rangle=-\langle\boldsymbol{P}|-i \delta H|\rangle^{*}=\frac{i g}{2 E^{3 / 2}} \mathcal{P}_{e \rightarrow \gamma e}^{*} \cdot \boldsymbol{P}_{31} \\
& \langle|-i \delta H| \boldsymbol{B}\rangle=-\langle\boldsymbol{B}|-i \delta H|\rangle^{*} \\
& =-\frac{g}{2 E^{3 / 2}} \mathcal{P}_{e \rightarrow \gamma e}^{*} \cdot \boldsymbol{\nabla} \delta^{(2)}\left(\boldsymbol{B}_{31}\right) \\
& \langle\boldsymbol{P}|+i \overline{\delta H}|\rangle=\langle\boldsymbol{P}|-i \delta H|\rangle_{(x, \boldsymbol{p}) \rightarrow(-x,-\boldsymbol{p})}^{*} \\
& =-\frac{i g}{2 E^{3 / 2}} \mathcal{P}_{e \rightarrow \gamma e}^{*} \cdot \boldsymbol{P}_{31} \\
& \langle\boldsymbol{B}|+i \overline{\delta H}|\rangle=\langle\boldsymbol{B}|-i \delta H|\rangle_{(x, \boldsymbol{b}) \rightarrow(-x, \boldsymbol{b})}^{*} \\
& \quad=-\frac{g}{2 E^{3 / 2}} \mathcal{P}_{e \rightarrow \gamma e}^{*} \cdot \boldsymbol{\nabla}^{(2)}\left(\boldsymbol{B}_{31}\right) \\
& \langle|+i \overline{\delta H}| \boldsymbol{P}\rangle=\langle\boldsymbol{P}|-i \delta H|\rangle^{*} \\
& \quad=-\frac{i g}{2 E^{3 / 2}} \mathcal{P}_{e \rightarrow \gamma e}^{*} \cdot \boldsymbol{P}_{31} \\
& \langle|+i \overline{\delta H}| \boldsymbol{B}\rangle=\langle\boldsymbol{B}|-i \delta H|\rangle^{*} \\
& =\frac{g}{2 E^{3 / 2}} \mathcal{P}_{e \rightarrow \gamma e}^{*} \cdot \boldsymbol{\nabla} \delta^{(2)}\left(\boldsymbol{B}_{31}\right) \\
& \left\langle\boldsymbol{P}^{\prime}|+i \overline{\delta H}| \boldsymbol{P}_{31}, \boldsymbol{P}_{42}\right\rangle \\
& \quad=\operatorname{previous} \times\left|x_{1}+x_{3}\right|^{-1}(2 \pi)^{2} \delta^{(2)}\left(\boldsymbol{P}_{42}^{\prime}-\boldsymbol{P}_{42}\right) \\
& \left\langle\boldsymbol{B}^{\prime}|+i \overline{\delta H}| \boldsymbol{B}_{13}, \boldsymbol{B}_{24}\right\rangle \\
& =\operatorname{previous} \times\left|x_{1}+x_{3}\right|^{-1} \delta^{(2)}\left(\boldsymbol{B}_{42}^{\prime}-\boldsymbol{B}_{42}\right)
\end{aligned}
$$

Figure 19. Variations on figure 17 that are related by complex conjugation and sometimes, depending on the directions drawn for momentum flow, by reversal $\left(x_{n}, \boldsymbol{p}_{n}\right) \rightarrow\left(-x_{n},-\boldsymbol{p}_{n}\right)$ of momentum variables (see appendix D for more detail). The overall factor of $\left|x_{1}+x_{3}\right|^{-1}=\left|x_{2}+x_{4}\right|^{-1}$ in the last diagram arises from the same effectively-2-particle state normalization factor for $\left|\boldsymbol{P}_{31}, \boldsymbol{P}_{42}\right\rangle$ that was discussed earlier in the text for the $\left\langle\boldsymbol{P}_{34}, \boldsymbol{P}_{12}\right|$ of figure 18. The two spectator lines in this case are colored black here to indicate that their color (red or blue) does not matter. [Note: the above vertex diagrams are best viewed in color.]

To illustrate the possible relations, we've shown more variations in figure 19 than we will actually need. The first line of vertex diagrams is not relevant for large $N_{\mathrm{f}}$, but at sub-leading order in $1 / N_{\mathrm{f}}$ would appear in the virtual-correction diagram of figure 20a. The second line of vertex diagrams would appear in the diagram for the complex conjugate of figure $7 \mathrm{a}$, shown in figure 20b. But this diagram need not be evaluated separately since we will be taking $2 \operatorname{Re}[\cdots]$ of the diagrams in figure 7 . The third and fourth lines of vertex diagrams in figure 19 appear in figures $8(\mathrm{i}, \mathrm{k}, \mathrm{m})$ and figures $8(\mathrm{~h}, \mathrm{l})$, respectively. Note that these vertices correspond to $(e \rightarrow \gamma e)^{*}$ in the conjugate amplitude and not to $e \bar{e} \rightarrow \gamma$. They are drawn as they are just because the diagrams for the amplitude and conjugate amplitude have been sewn together. Depending on your taste, it might have been clearer to draw the third line of figure 19 as figure 20c, but we have chosen to keep with the same style of drawings as in ref. [7] (AI1).

Using these rules, and the additional vertex rules of appendix $\mathrm{D}$, the calculation of the real double splitting diagrams of figure 7 proceeds almost the same as our gluon-splitting calculations in our earlier work [7-10]. In particular, the calculation of the second line of diagrams in figure 7 , which involve the instantaneous 4 -fermion vertex of LCPT, is very similar to the calculation of QCD diagrams involving the 4-gluon vertex [10]. Because 


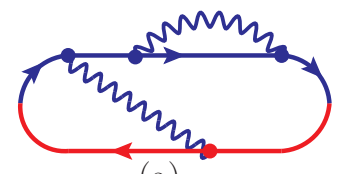

(a)

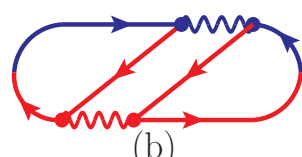

(b)

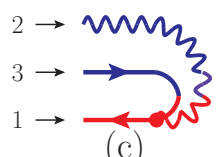

(c)

Figure 20. (a) A virtual correction not considered in this paper because it is sub-leading in $1 / N_{\mathrm{f}}$. (b) The complex conjugate of figure 7a. (c) An alternative way that we could have drawn the vertex in the third line of diagrams in figure 19, which would have made it clearer that the vertex correspond to $e \rightarrow \gamma e$ in the conjugate amplitude.

these calculations are so close to previous work, we leave the details and analytic results to appendix E.

We'll mention just one qualitative detail here: during effectively 2-particle evolution (e.g. the middle shaded region of figure 11b), the system evolves in medium like a coupled pair of 2-dimensional non-Hermitian harmonic oscillators, with two complex eigenfrequencies. ${ }^{16}$ In (large- $N_{\mathrm{c}}$ ) QCD double bremsstrahlung, both eigenfrequencies are non-zero. In QED, however, one vanishes. See appendix E for details, but this difference does not have any particular impact on the method of calculation.

\subsection{An implicit approximation}

There was an implicit approximation made in the above treatment of instantaneous LCPT interactions. Remember that the high-energy particles shown in our diagrams, such as figure 7 , are implicitly interacting many times with the medium. We should take a minute to think about what these diagrams would look like if we explicitly drew all the interactions with the soft particles in the medium. In particular, let's think about what happens to the longitudinal intermediate photon lines in the second row of diagrams in figure 7 . In principle, these could be dressed by soft interactions as in figure 21a. (Note that we've been careful not to add any new high-energy particles to the final state, because otherwise the figures we have drawn previously would not have been complete drawings of all the high-energy particles.) In the language of thermal field theory Feynman diagrams, the contribution from figure 21a would be captured by dressing the photon propagator with thermal loops as in figure 21b. These soft interactions have not been included in the vertex rule we have given in figure 18: the formulas there were based on the vacuum LCPT rule given inside the box.

Fortunately, medium corrections to the vertex rule of figure 18 will be suppressed by some power of the high energy scale $E$. The typical transverse separation $b$ of the high-energy particles during an LPM formation time are of order

$$
b \sim \frac{1}{Q_{\perp}} \sim \sqrt{\frac{t_{\text {form }}}{E}} \sim \frac{1}{(\hat{q} E)^{1 / 4}}
$$

(suppressing dependence on longitudinal momentum fractions, and working in the thickmedia limit being considered in this paper). In the limit of large $E$, this is parametrically

\footnotetext{
${ }^{16}$ See AI1 section V.B [7]. Two complex frequencies $\Omega$ also appear in the earlier work of refs. [4-6] on double, small- $x$ gluon bremsstrahlung, where each $\Omega$ is determined by one of the two bremsstrahlung gluon energies.
} 


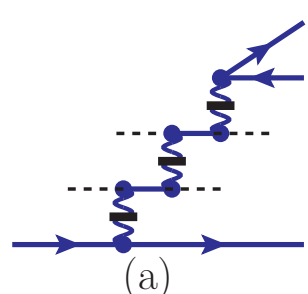

(a)

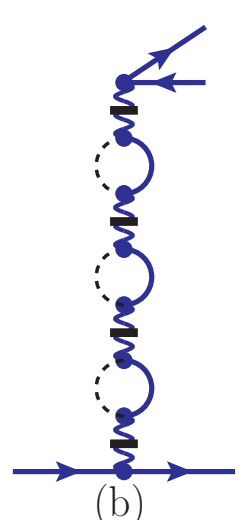

(b)

Figure 21. (a) Possible soft medium corrections to the instantaneous longitudinal photon interaction of LCPT. Dashed lines represent soft electrons or positrons; intermediate solid lines represent intermediate high-energy particles. (b) How such corrections might alternatively be drawn in the language of thermal or finite-density loops. [(b) is expressed here as a Feynman diagram rather than a time-ordered diagram.]
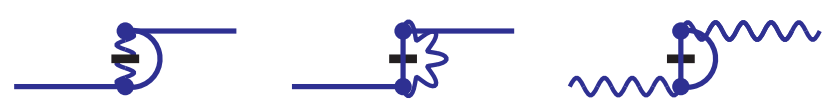

Figure 22. Additional 2-point LCPT interactions arising from normal ordering the instantaneous interactions of figure 15 .

small compared to any distance scale that characterizes the medium, such as the typical distance between medium particles. So, the chance that there is a medium interaction during the instant of the instantaneous interaction is parametrically small and can be ignored. ${ }^{17}$

On a similar note, there are some other types of interactions in time-ordered LCPT calculations that we have also ignored. The LCPT rules that we have quoted assume that the LCPT Hamiltonian has been normal ordered. When normal ordering the instantaneous interactions, such as figure 15, there are contractions that produce additional 2-point interactions in the normal-ordered Hamiltonian, which are often depicted diagrammatically [13] as in figure 22. In our large- $N_{\mathrm{f}}$ QED calculation, these can contribute to the virtual correction to $e \rightarrow \gamma e$ as in figure 23, which should be added to the diagrams we listed earlier in figure 9. Fortunately, we can ignore these additional diagrams for reasons somewhat similar to those for the LCPT analysis of NLO deep inelastic scattering in refs. [22, 24]. Because we are in the high-energy limit, we have ignored the masses of our high-energy particles. We will be using dimensional regularization, and normal-ordering contractions such as figure 22 vanish in dimensional regularization for massless particles in vacuum, which is ultimately a consequence of dimensional analysis. Unlike refs. [22, 24], however, our loops are not in vacuum, and the medium introduces a scale that in principle invalidates the argument that these loops will vanish. But because these particular loops involve an instantaneous interaction, and because transverse separations are suppressed by powers

\footnotetext{
${ }^{17}$ In contrast, the effect of the medium on the evolution between vertex times in our diagrams (e.g. the shaded regions of figure 11) cannot be ignored because the times between these vertices are of order formation times, which are parametrically large in the high energy limit.
} 


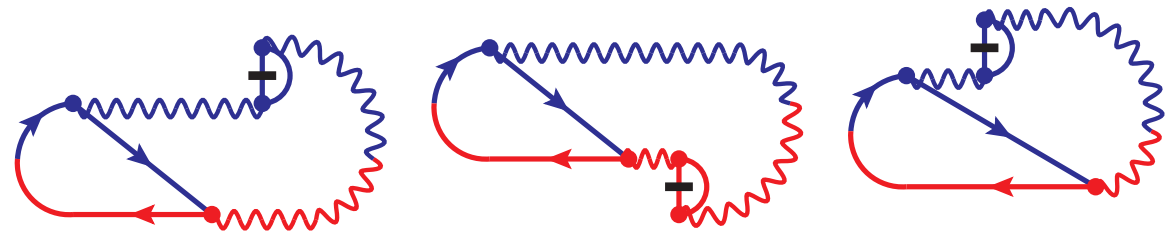

Figure 23. Additional virtual diagrams for an (in-medium) LCPT calculation of $e \rightarrow \gamma e$, which should in principle be added to figure 8 but which are ignorable in our calculation.

of the energy $E$, the effect of the medium on the loops of figure 22 are negligible in the high-energy limit. So we may ignore the diagrams of figure 23.

\section{Virtual corrections to single splitting}

Consider the diagrams of figure 8, which represent next-to-leading order corrections to single-splitting. Each of these diagrams contain a virtual $e \bar{e}$ loop. We shall now see that almost all of these diagrams are related in a relatively simple way to the double-splitting diagrams of figure 7. As mentioned earlier, there is one exception: the boxed diagram of figure $8 \mathrm{k}$, which will require a new (and quite non-trivial) calculation. But let's start with the simpler cases.

\subsection{Back-end transformation}

Most of the diagrams of figure 8 (namely $h, i, j, l, n$ ) are related to various double emission diagrams of figure 7 (a,b,c,e,g respectively) by a simple diagrammatic procedure: take the latest-time vertex in the diagram, and slide it around the right end of the diagram from being a vertex in the amplitude to being a vertex in the conjugate amplitude, or vice versa. For concreteness, a specific example is shown in figure 24. We will call this a "back-end" transformation. Provided that the vertex being moved is the latest-time vertex in both diagrams, there is an extremely simple relationship between the values of the diagrams: before integrating over any longitudinal momentum fractions, the two diagrams differ only by an overall minus sign. Heuristically, this minus sign can be roughly understood from the relation of virtual loops, through the optical theorem, to the probability of something not happening. So, for example, figures $7(\mathrm{~b}, \mathrm{c})$ are, roughly speaking, related to the probability $P_{2}$ of one splitting $e \rightarrow \gamma e$ later being followed by another, $\gamma \rightarrow e \bar{e}$. Figures $8(\mathrm{i}, \mathrm{j})$, on the other hand, are roughly related to the probability $P_{1}$ of one splitting $e \rightarrow \gamma e$ not being later followed by another. ${ }^{18}$ Any increase in $P_{2}$ should be accompanied by a decrease in $P_{1}$ by conservation of probability, and so one may expect these diagrams to be the same

\footnotetext{
${ }^{18}$ This characterization makes sense for (1) a formal calculation of probabilities in the limit where the medium has some finite (but very large) size $L$, formally expanding to second-order in perturbation theory in hard splittings. That is, the limit $\alpha \rightarrow 0$ for fixed $L$ and fixed particle energies (with $L$ large compared to formation times and $\alpha$ here referring to the $\alpha$ at the scale characterizing the high-energy splitting). More precisely, this is the limit where the mean free time between splittings is large compared to $L$. But one may be interested in the opposite order of limits (2) $L \rightarrow \infty$ with fixed $\alpha \ll 1$ and fixed particle energies that is, the case where the mean free time between splittings is small compared to $L$. As discussed in detail in ACI2 [8], the formal calculation (1) can be used to figure out a correction to splitting probabilities that allows for the calculation (2), and so it is in the formal context of (1) that we always discuss our diagrams, even for those cases where our ultimate interest may be (2).
} 


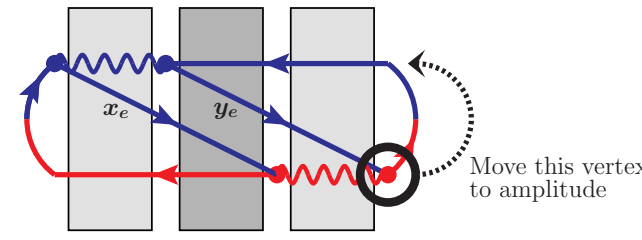

diagram (a)

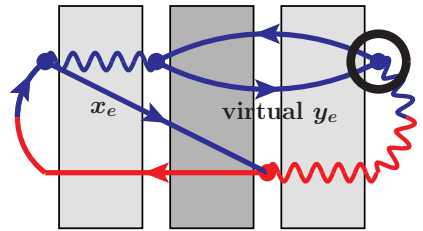

diagram $(\mathrm{h})$

Figure 24. Example of two diagrams related by a back-end transformation, in which the final vertex in one diagram is slid around the right-hand side (back end) of the diagram to become the final vertex of the other diagram. We've shaded the evolution between vertices as in figure 11, just to emphasize that the general pattern of these evolutions does not change.

size but with opposite signs. (In principle, the total probability that's actually conserved at this order is $P_{0}+P_{1}+P_{2}$, where $P_{0}$ is the probability that neither emission occurs. But the cases of the diagrams that we are relating by back-end transformations are cases where changes to $P_{0}$ do not come into play.)

By writing expressions for diagrams that are related by a back-end transformation, using the vertex rules of section 3.1 and appendix D, and then tying them together with $n$-particle evolution in the medium as in figure 11 and refs. [7, 8] (AI1,ACI2), one may verify that back-end transformations really are that simple: there is just an overall sign difference. Note in particular that the longitudinal momentum fractions of the particles are the same in the two diagrams of figure 24 , for each of the three shaded regions of in-medium evolution. So those evolution factors match up identically between the two diagrams.

Virtual loops should be integrated over the longitudinal momenta of the particles in the loop. So the final relationship between the pairs of back-end related diagrams discussed above can be written as

$$
\left[\frac{d \Gamma}{d x_{e}}\right]_{(\mathrm{h}, \mathrm{i}, \mathrm{j}, \mathrm{l}, \mathrm{n})}=-\int_{0}^{1-x_{e}} d y_{e}\left[\frac{d \Gamma}{d x_{e} d y_{e}}\right]_{(\mathrm{a}, \mathrm{b}, \mathrm{c}, \mathrm{e}, \mathrm{g})},
$$

where $\Gamma$ is the rate, $x_{e}$ is the momentum of the daughter whose electron line is connected to the original electron, and $y_{e}$ is the other electron in the final state of real double splitting processes $e \rightarrow e \bar{e} e$, as in figure 24. The integration limits come from the facts that (i) the longitudinal momentum fractions in the produced $e \bar{e}$ pair are $y_{e}$ and $1-x_{e}-y_{e}$ in these diagrams (relative to the initial electron), and (ii) longitudinal momentum fractions are always positive in LCPT.

Thinking about the UV divergences of loop diagrams vs. tree diagrams, one might be uneasy with the idea that diagrams (a) and (h) in figure 24 could possibly be related so simply. In particular, diagram (h) involves a photon self-energy, and photon self-energies are UV divergent. ${ }^{19}$ In contrast, diagram (a) represents an interference term that contributes

\footnotetext{
${ }^{19}$ The back-end transformation says that diagrams (a) and (h) differ by an overall sign before integrating (h) over $y_{e}$. In LCPT, the range of integration in (4.1) for $y_{e}$ is finite ( $y_{e}$ is not integrated to infinity) because both virtual particles in the loop are forced to have positive longitudinal momentum $p^{+}$. So any UV divergence will already be present even before integrating over $y_{e}$.
} 
to the calculation of the magnitude-square of a tree-level amplitude, and tree-level rates are not UV divergent. Nonetheless, diagram (a) by itself is UV divergent, even though those divergences cancel in the sum $2 \operatorname{Re}(\mathrm{a}+\mathrm{b}+\mathrm{c})$ of such interference terms, drawn in figure $7 .^{20}$ This is a general issue with individual time-ordered (as opposed to Feynman) diagrams. For the same reason, the UV divergence of an individual virtual-correction diagram like diagram (h) is different from merely the UV divergence one associates with a photon selfenergy, because diagram $(\mathrm{h}$ ) has a time-order constraint that the emission in the conjugate amplitude occur during the electron pair fluctuation of the photon. Because the sum of virtual diagrams $2 \mathrm{Re}(\mathrm{h}+\mathrm{i}+\mathrm{j})$ of figure 8 is related by back-end transformation to the sum of diagrams $2 \operatorname{Re}(a+b+c)$, and because UV divergences cancel in the sum $2 \operatorname{Re}(a+b+c)$, as they must, this means that all the UV divergences of the sum $2 \operatorname{Re}(\mathrm{h}+\mathrm{i}+\mathrm{j})$ must cancel as well, including UV divergences associated with the photon self-energy. That leaves diagram (k) as the only uncanceled UV divergence among the first line of figure 8 (and it turns out to be the only uncanceled UV divergence among all the diagrams). Reassuringly, we will find that the time ordering represented by diagram (k), by itself, indeed gives exactly the right amount of divergence to produce, in our calculation, the known renormalization of $\alpha_{\mathrm{EM}}$.

\subsection{Front-end transformations}

There is a somewhat related relation between pairs of diagrams where we instead take the earliest-time vertex in a diagram and slide it around the left end of the diagram to move it from the amplitude to the conjugate amplitude or vice versa. We will refer to this as a front-end transformation, an example of which is shown in figure 25. We'll discuss how to implement a front-end transformation in a moment, but first note its utility. Using a front-end transformation, along with complex conjugation as necessary, the $\gamma \rightarrow$ $e \bar{e}$ virtual correction diagrams of figures $9(\mathrm{o}, \mathrm{p}, \mathrm{q}, \mathrm{s}, \mathrm{u})$ can be related to the real double bremsstrahlung diagrams of figures $7(\mathrm{a}, \mathrm{b}, \mathrm{c}, \mathrm{f}, \mathrm{g})$, respectively. Using both a front-end and a back-end transformation on a single diagram, figures $8(\mathrm{~m})$ and $9(\mathrm{t})$ can be related to figures $7(\mathrm{e}, \mathrm{f})$, respectively, and figure $9(\mathrm{r})$ can be related to figure $8(\mathrm{k})$. In consequence, the only virtual diagram calculation that we will need to do from scratch is the boxed diagram of figure $8(\mathrm{k})$ : an (in-medium) photon self-energy loop in the "middle" of a single splitting $e \rightarrow \gamma e$.

Front-end transformations are more complicated to implement than back-end ones. If we were to simply slide the vertex around as in figure 25 while keeping the labeling of all longitudinal momenta the same, we would get the transformation shown by the first two diagrams of figure 26. There are two problems with the middle diagram in figure 26, which is supposed to represent a virtual correction to $\gamma \rightarrow e \bar{e}$. The first problem is that

\footnotetext{
${ }^{20}$ On the technical side, see the discussion of " $\Delta t \rightarrow 0$ " UV divergences and their cancellation for QCD diagrams similar to our $(a+b+c)$ here in sections II.A.4 and II.B.2 of ref. [8], or the earlier discussion for a different set of QCD diagrams in ref. [7]. Also, our language is somewhat loose above. When we refer to the sum $2 \operatorname{Re}(\mathrm{a}+\mathrm{b}+\mathrm{c})$ we actually mean the difference between (i) that sum and (ii) what one would have gotten by instead treating the process as two successive, independent single splittings each calculated using leading-order formulas for single spitting rates. In our notation here and elsewhere, we call this difference " $\Delta d \Gamma / d x d y . "$ Physically, $\Delta d \Gamma / d x d y$ corresponds to the correction to double emission due to overlap effects. See sections I.A and II.A of ref. [8].
} 


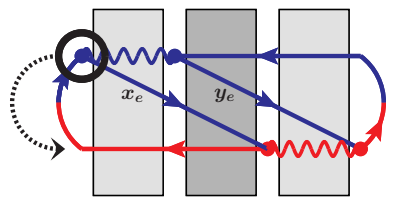

diagram (a)

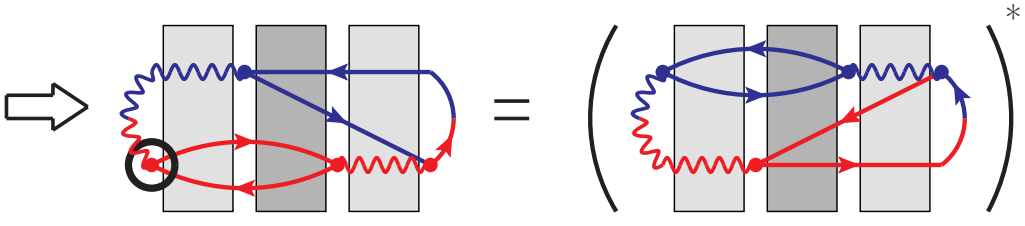

$(\operatorname{diagram}(\mathrm{o}))^{*}$

Figure 25. Like figure 24 but for a "front-end" transformation.
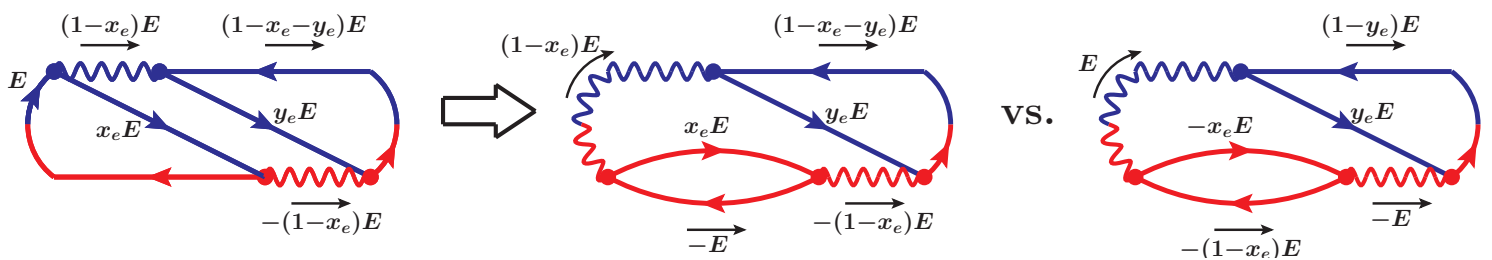

Figure 26. The first diagram shows the same contribution $e \rightarrow e \bar{e} e$ as in figure 25, but here we have labeled all longitudinal momenta. The second diagram shows what would happen if we slid the initial vertex around as in the front-end transformation of figure 25 without changing the labeling of longitudinal momenta. For comparison, the last diagram shows the actual labeling of longitudinal momenta for this $\gamma \rightarrow e \bar{e}$ process with an initial photon of energy $E$. In all of these diagrams, momenta are taken to flow in the direction of fermion-line arrows unless indicated otherwise by a small black arrow. This generally corresponds to labeling the momenta as flowing from left to right in the interference diagram.

our convention is always to let $E$ refer to the energy of the original high-energy particle in any process, which in this case would be the photon. The second is that $x_{e}$ is positive in the diagram of figure 26, which is then inconsistent with the fact that the longitudinal momentum $x_{e} E$ shown in the second diagram should be negative, given our convention that longitudinal momenta of conjugate-amplitude particles are negative in our interference diagrams. This second problem can also be visualized by comparing the earlier figures 24 and 25 for back-end and front-end transformations. In the back-end case of figure 24, both the original diagram and the transformed diagram had exactly the same particles evolving in each of the three shaded regions. In the front-end case given by the first two diagrams of figure 25, the last shaded region is the same, but an "amplitude" particle (blue line) has been switched to a "conjugate amplitude" particle (red line) in each of the first two shaded areas. That means that the evolution in those shaded areas is not exactly the same for those two diagrams, and so their relation is not as simple as it was for a back-end transformation.

There is a simple way to overcome both obstacles, which is to make the change of variables

$$
\left(x_{e}, y_{e}, E\right) \rightarrow\left(\frac{-x_{e}}{1-x_{e}}, \frac{y_{e}}{1-x_{e}},\left(1-x_{e}\right) E\right)
$$

when making a front-end transformation like figure 25 . This converts the momenta of the middle diagram of figure 26 into those of the last diagram. It also negates the value of $x_{e}$. As long as one writes formulas for evolution in diagrams in a way that is general enough to 


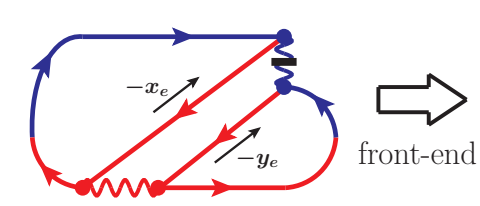

$\operatorname{diagram}(\mathrm{f})$

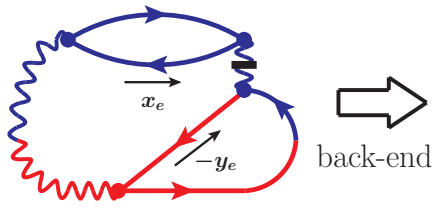

diagram (s)
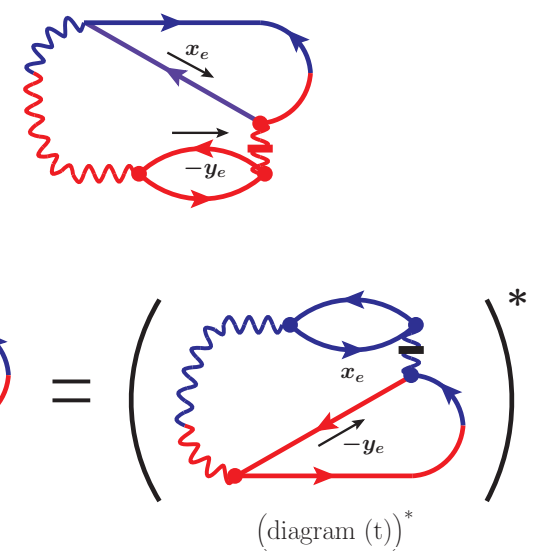

Figure 27. The transformation of diagram (f) to diagram (t) by front- and back-end transformations, relabeling momentum fractions, and conjugation.

correctly handle the cases of both positive or negative longitudinal momentum fractions, similar to ref. [7] (AI1), then all will be well. The transformation (4.2) is its own inverse, and so works just as well transforming $\gamma \rightarrow e \bar{e}$ back again to $e \rightarrow e \bar{e} e$. In terms of the diagram labels in figures 7 and 9 ,

$$
2 \operatorname{Re}\left[\frac{d \Gamma}{d y_{e}}\right]_{(\mathrm{o}, \mathrm{p}, \mathrm{q}, \mathrm{s}, \mathrm{u})}=-2 N_{\mathrm{f}} \operatorname{Re} \int_{0}^{1} d x_{e}\left\{\left[\frac{d \Gamma}{d x_{e} d y_{e}}\right]_{(\mathrm{a}, \mathrm{b}, \mathrm{c}, \mathrm{f}, \mathrm{g})} \text { with substitutions (4.2) }\right\}
$$

is the front-end analog of $(4.1) .{ }^{21}$ Here we have taken $2 \operatorname{Re}[\cdots]$ of all the diagrams, which we must do anyway at the end of the calculation, and which here obviates the need to specify exactly which diagrams need to be complex conjugated after the front-end transformation. Note that we have written the $\gamma \rightarrow e \bar{e}$ rate as $d \Gamma / d y_{e}$ to conform with our convention that the electron produced by pair production is labeled $y_{e}$, as in the last diagram of figure 26 . The relative factor $N_{\mathrm{f}}$ in (4.3) just reflects the fact that one of the electron flavors in $e \rightarrow e \bar{e} e$ is fixed to be that of the initial electron but there is no similar flavor constraint for NLO $\gamma \rightarrow e \bar{e}$.

Performing both a front-end and back-end transformation relates

$$
2 \operatorname{Re}\left[\frac{d \Gamma}{d y_{e}}\right]_{(\mathrm{t})}=+2 N_{\mathrm{f}} \operatorname{Re} \int_{0}^{1} d x_{e}\left\{\left[\frac{d \Gamma}{d x_{e} d y_{e}}\right]_{(\mathrm{f})} \quad \begin{array}{l}
\text { with substitutions }(4.2) \\
\text { followed by }\left(x_{e}, y_{e}\right) \rightarrow\left(1-y_{e}, x_{e}\right)
\end{array}\right\} .
$$

As shown in figure 27, the additional step of $\left(x_{e}, y_{e}\right) \rightarrow\left(1-y_{e}, x_{e}\right)$ is needed to make the labeling of the momentum fractions in diagram ( $\mathrm{t}$ ) match up with our conventions.

A different variant of the front-end transformation is needed for interference diagrams whose earliest vertex is an instantaneous 4-fermion interaction, such as figure 7(e). Figure 28 shows diagrammatically the difference between (i) "simply sliding the vertex

\footnotetext{
${ }^{21}$ The change of $E$ to $\left(1-x_{e}\right) E$ in (4.2) might have also led to some power of $1-x_{e}$ appearing as an overall normalization factor in (4.3). However, for the front-end transformation of $d \Gamma / d x_{e} d y_{e}$ here, there turns out to be no such factor in four spacetime dimensions. (See appendix H.2 for a more general discussion.)
} 

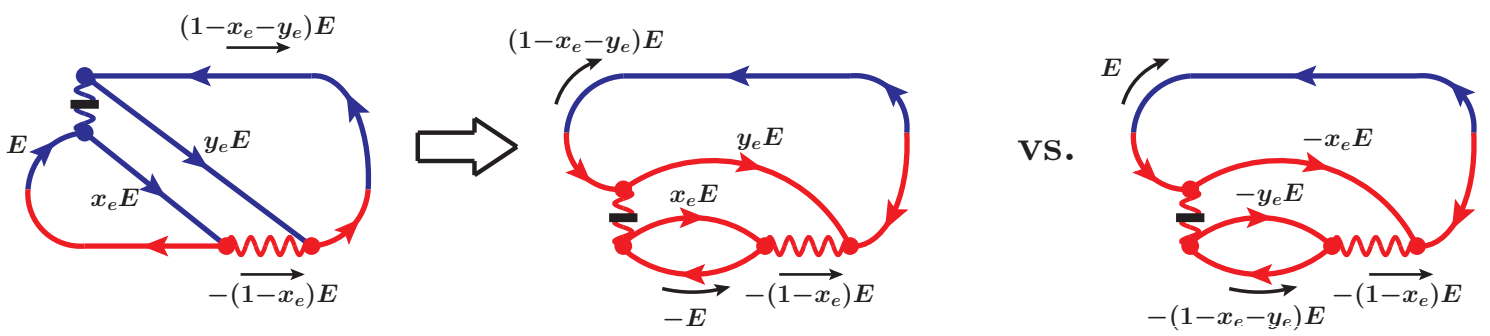

Figure 28. A front-end transformation somewhat like figure 26 but for a diagram whose earliesttime vertex is instead an instantaneous 4 -fermion interaction. The last diagram above is related by back-end transformation to the complex conjugate of figure $8(\mathrm{~m})$.

around" while keeping the labeling the same vs. (ii) the actual result we want to achieve with a front-end transformation. We can implement the necessary relabeling of momenta by making the change of variables

$$
\left(x_{e}, y_{e}, E\right) \rightarrow\left(\frac{-y_{e}}{1-x_{e}-y_{e}}, \frac{-x_{e}}{1-x_{e}-y_{e}},\left(1-x_{e}-y_{e}\right) E\right)
$$

somewhat similar to (4.2). Performing both a front-end and back-end transformation then relates diagrams $(\mathrm{m})$ and $(\mathrm{e})$ as

$$
2 \operatorname{Re}\left[\frac{d \Gamma}{d x_{e}}\right]_{(\mathrm{m})}=+2 \operatorname{Re} \int_{0}^{1-x_{e}} d y_{e}\left\{\left[\frac{d \Gamma}{d x_{e} d y_{e}}\right]_{(\mathrm{e})} \text { with substitutions (4.5) }\right\} .
$$

\subsection{The fundamental virtual diagram}

\subsubsection{Overview}

As previously discussed, the one virtual correction diagram which cannot be related to the $e \rightarrow e \bar{e} e$ diagrams is figure 29, which is a more detailed version of figure 8k. Following notation similar to that of ref. [7] (AI1), we will refer to this as the $x y y \bar{x}$ diagram since it involves, in order, (i) emission of an $x_{e}$ electron in the amplitude, (ii) emission of a $y_{e}$ electron in the amplitude, (iii) re-absorption of the $y_{e}$ electron in the amplitude, followed finally by (iv) emission of the $x_{e}$ electron in the conjugate amplitude. This virtual correction diagram contributes

$$
\left[\frac{d I}{d x_{e}}\right]_{x y y \bar{x}}=\int_{0}^{1-x_{e}} d y_{e}\left[\frac{d I}{d x_{e} d y_{e}}\right]_{x y y \bar{x}}
$$

to the differential emission probability for $e \rightarrow \gamma e$, where, adapting the notation of refs. $[7,8]$ (AI1,ACI2),

$$
\begin{aligned}
{\left[\frac{d I}{d x_{e} d y_{e}}\right]_{x y y \bar{x}}=\left(\frac{E}{2 \pi}\right)^{2} } & \int_{t_{\mathrm{x}}<t_{\mathrm{y}}<t_{\mathrm{y}^{\prime}}<t_{\overline{\mathrm{x}}}} \sum_{\text {pol. }}\left\langle|i \overline{\delta H}| \boldsymbol{B}^{\overline{\mathrm{x}}}\right\rangle\left\langle\boldsymbol{B}^{\overline{\mathrm{x}}}, t_{\overline{\mathrm{x}}} \mid \boldsymbol{B}^{\mathrm{y}^{\prime}}, t_{\mathrm{y}^{\prime}}\right\rangle \\
& \times\left\langle\boldsymbol{B}^{\mathrm{y}^{\prime}}|-i \delta H| \boldsymbol{C}_{41}^{\mathrm{y}^{\prime}}, \boldsymbol{C}_{23}^{\mathrm{y}^{\prime}}\right\rangle\left\langle\boldsymbol{C}_{41}^{\mathrm{y}^{\prime}}, \boldsymbol{C}_{23}^{\mathrm{y}^{\prime}}, t_{\mathrm{y}^{\prime}} \mid \boldsymbol{C}_{41}^{\mathrm{y}}, \boldsymbol{C}_{23}^{\mathrm{y}}, t_{\mathrm{y}}\right\rangle \\
& \times\left\langle\boldsymbol{C}_{41}^{\mathrm{y}}, \boldsymbol{C}_{23}^{\mathrm{y}}|-i \delta H| \boldsymbol{B}^{\mathrm{y}}\right\rangle\left\langle\boldsymbol{B}^{\mathrm{y}}, t_{\mathrm{y}} \mid \boldsymbol{B}^{\mathrm{x}}, t_{\mathrm{x}}\right\rangle\left\langle\boldsymbol{B}^{\mathrm{x}}|-i \delta H|\right\rangle .
\end{aligned}
$$



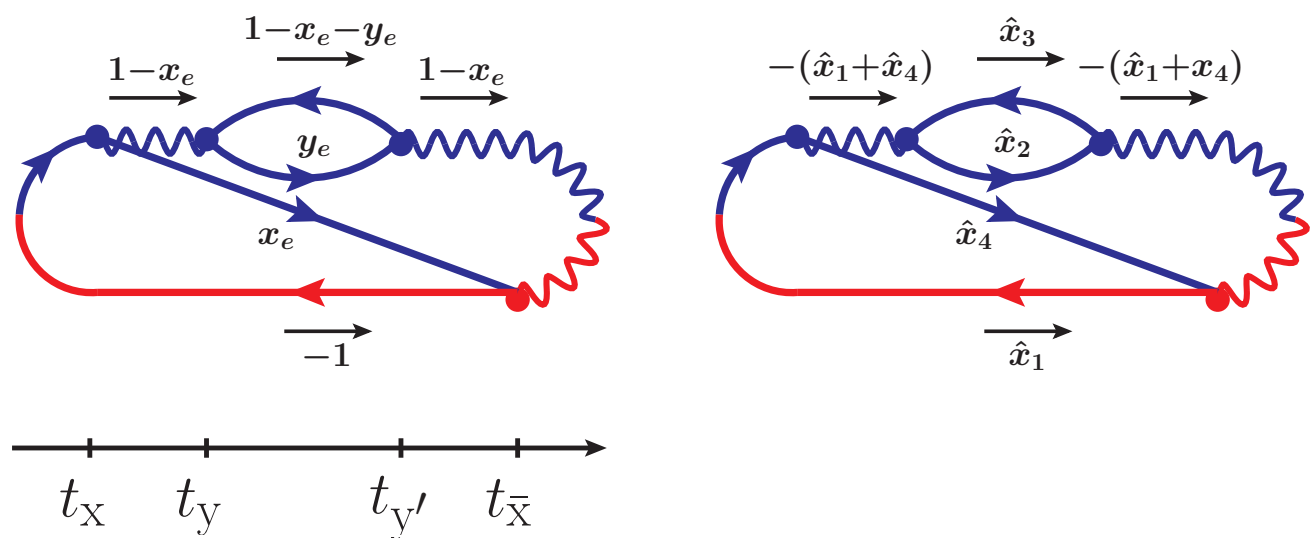

Figure 29. The basic virtual diagram $x y y \bar{x}$ of figure $8 \mathrm{k}$, here with longitudinal momentum fractions labeled in terms of $x_{e}$ and $y_{e}$ [left] or the labels $\hat{x}_{i}$ (4.9) [right]. Our notation for vertex times is also shown.

In broad outline, this formula simply convolves the relevant vertex matrix elements at different vertex times with medium-averaged propagators between those times. ${ }^{22}$ The notation $\boldsymbol{C}_{i j}$ refers to the $\boldsymbol{B}_{i j} \equiv\left(\boldsymbol{b}_{i}-\boldsymbol{b}_{j}\right) /\left(x_{i}+x_{j}\right)$ variables defined in (3.4); we use the letter $\boldsymbol{C}$ just to help distinguish $\boldsymbol{B}_{i j}$ variables used in the context of 4-particle (effectively 2-particle) evolution. The indices on $\boldsymbol{C}_{i j}$ refer to the particles involved in the 4-particle evolution according to the convention of the right-hand drawing in figure 29, for which we introduce the labeling [7]

$$
\left(\hat{x}_{1}, \hat{x}_{2}, \hat{x}_{3}, \hat{x}_{4}\right) \equiv\left(-1, y_{e}, 1-x_{e}-y_{e}, x_{e}\right)
$$

of longitudinal momentum fractions.

Initially, the evaluation of (4.8) proceeds very similarly to that of other diagrams, following refs. [7, 8] (AI1,ACI2). One puts in explicit formulas for the $\delta H$ matrix elements and for the time-evolution factors. It is then possible to analytically perform all the integrations implicit in (4.8) over the intermediate transverse positions specified by the $\boldsymbol{B}$ 's and $C$ 's, as well as all but one of the time integrations. Due to time translation invariance, one combination of those time integrations just gives a factor of total time $\mathbb{T}$. After all these integrations, the differential rate $d \Gamma \equiv d I / \mathbb{T}$ corresponding to (4.8) may be reduced to a single remaining integral over the time difference $\Delta t$ between the two intermediate vertex times in the process under consideration. Schematically,

$$
\left[\frac{d I}{d x_{e} d y_{e}}\right]_{x y y \bar{x}}=\int_{0}^{\infty} d(\Delta t) F(\Delta t) .
$$

For the $x y y \bar{x}$ process of figure $29, \Delta t \equiv t_{\mathrm{y}^{\prime}}-t_{\mathrm{y}}$ is the duration of the photon self-energy loop. The integrand $F(\Delta t)$ has a somewhat complicated formula (schematically similar to other diagrams), which we leave to appendix F. Here, in the main text, our goal is to give a broad overview of what is involved in dealing with the UV divergence of the $\Delta t$ integral

\footnotetext{
${ }^{22}$ In particular, compare and contrast to AI1 (4.10) [7].
} 
in (4.10). To be concrete: for small $\Delta t$, the integrand turns out (see appendix F.2) to have non-integrable divergences

$$
F(\Delta t) \propto \frac{\ln \left(2 i \Omega_{\mathrm{i}} \Delta t\right)}{(\Delta t)^{2}}-\frac{i \Omega_{\mathrm{i}}}{\Delta t} \quad\left(\text { for } \Delta t \ll\left|\Omega_{\mathrm{i}}\right|^{-1}\right),
$$

where the magnitude of

$$
\Omega_{\mathrm{i}}=\sqrt{-\frac{i\left(1-x_{e}\right) \hat{q}}{2 x_{e} E}}
$$

can be interpreted physically as the scale of the QED inverse formation time (2.4) for leading-order $e \rightarrow \gamma e$.

UV divergences from $\Delta t \rightarrow 0$ in the calculation of time-ordered interference diagrams are not restricted just to loop diagrams like figure 29. Individually, the real double splitting diagrams of figure $7(\mathrm{a}-\mathrm{c})$ for $e \rightarrow e \bar{e} e$ also have $\Delta t \rightarrow 0$ divergences when written in the form $\int d(\Delta t) F(\Delta t)$, analogous to the detailed discussion of the QCD case in refs. [7-9] (AI1ACI3). Those divergences cancel between the three diagrams of figure $7(\mathrm{a}-\mathrm{c})$, also similar to refs. [7-9]. Consistently evaluating the remaining finite part is a little tricky and was accomplished [9] by computing the individual diagrams using dimensional regularization.

Because the virtual diagrams of figure $8(\mathrm{~h}-\mathrm{j})$ are related to figure $7(\mathrm{a}-\mathrm{c})$ by a simple back-end transformation (4.1), the divergences of $(\mathrm{h}-\mathrm{j})$ will also cancel. Additionally, we find that diagrams involving instantaneous longitudinal photon interactions are all individually finite. That means that the only uncanceled UV divergence in our calculations of $e \rightarrow e \bar{e} e$ and $e \rightarrow \gamma e$ is the one for the $x y y \bar{x}$ diagram shown in both figures $7(\mathrm{k})$ and 29. [The divergence of the $\gamma \rightarrow e \bar{e}$ diagram of figure 9(r) is similarly uncanceled, but, as already noted, that diagram is related to the other by front- and back-end transformations.]

Unfortunately, our previous method [9] for calculating diagrams in dimensional regularization breaks down for the $x y y \bar{x}$ diagram, for reasons that will be explained later. We will leave the (we think interesting) details to appendices. Here we convey the gist of the method.

\subsubsection{Dimensional regularization: strategy}

In this paper, the symbol $d \equiv d_{\perp}$ will represent the number

$$
d=2-\epsilon
$$

of transverse dimensions, not the total number $4-\epsilon$ of space-time dimensions. It might reduce the opportunity for confusion if we always wrote our $d$ as $d_{\perp}$, but we drop the subscript to save space in what will be some complicated equations.

We do not know how to do the entire integral (4.10) for arbitrary $d$. Fortunately, we do not have to. The only divergence comes from $\Delta t \rightarrow 0$, and so we only need to regulate the small- $\Delta t$ portion of the integral. Let $a$ be an arbitrarily small but finite time scale, and split (4.10) up into

$$
\int_{0}^{\infty} d(\Delta t) F(\Delta t)=\int_{0}^{a} d(\Delta t) F(\Delta t)+\int_{a}^{\infty} d(\Delta t) F(\Delta t) .
$$


We only need dimensional regularization for the first term on the right-hand side. Specifically,

$$
\int_{0}^{\infty} d(\Delta t) F_{d}(\Delta t)=\int_{0}^{a} d(\Delta t) F_{d}(\Delta t)+\int_{a}^{\infty} d(\Delta t) F_{2}(\Delta t)+O(\epsilon),
$$

where $F_{d}(\Delta t)$ is the integrand for $d$ transverse dimensions. Roughly speaking, our goal is to use small- $\Delta t$ approximations to do the $\Delta t<a$ integral analytically, and then use numerical integration to do the $\Delta t>a$ integral. But there is a problem: as we take $a \rightarrow 0$ (in order to make our calculation of the first integral arbitrarily accurate), the second integral $\int_{a}^{\infty} F_{2}(\Delta t)$ will blow up, which is undesirable for numerical integration. We can isolate this problem by rewriting the right-hand side of (4.15) as

$$
\int_{0}^{a} d(\Delta t) F_{d}(\Delta t)+\int_{a}^{\infty} d(\Delta t) \mathcal{D}_{2}(\Delta t)+\int_{a}^{\infty} d(\Delta t)\left[F_{2}(\Delta t)-\mathcal{D}_{2}(\Delta t)\right]+O(\epsilon),
$$

where $\mathcal{D}_{2}(\Delta t)$ is any convenient function that

- matches the divergence of $F_{2}(\Delta t)$ as $\Delta t \rightarrow 0$ [which is proportional to (4.11)];

- falls off fast enough as $\Delta t \rightarrow \infty$ so that $\int_{a}^{\infty} d(\Delta t) \mathcal{D}_{2}(\Delta t)$ will converge for non-zero $a$;

- is simple enough that $\int_{a}^{\infty} d(\Delta t) \mathcal{D}_{2}(\Delta t)$ can be performed analytically.

The last integral in (4.16) is then convergent by itself in the $a \rightarrow 0$ limit, and so we may replace (4.16) by

$$
\lim _{a \rightarrow 0 "}\left[\int_{0}^{a} d(\Delta t) F_{d}(\Delta t)+\int_{a}^{\infty} d(\Delta t) \mathcal{D}_{2}(\Delta t)\right]+\int_{0}^{\infty} d(\Delta t)\left[F_{2}(\Delta t)-\mathcal{D}_{2}(\Delta t)\right]+O(\epsilon) .
$$

Our strategy will be to evaluate the first two integrals in (4.17) analytically in the limit of small $a$ (and verify cancellation of the $a$ dependence) and to evaluate the (convergent) third integral numerically. The choice of $\mathcal{D}_{2}(\Delta t)$ is not unique, but the final answer for (4.17) will be independent of the details of that choice. The particular choice we will find convenient is

$$
\mathcal{D}_{2}(\Delta t) \propto \frac{\ln \left(2 i \Omega_{\mathrm{i}} \Delta t\right)}{(\Delta t)^{2}}-i \Omega_{\mathrm{i}}^{3} \Delta t \csc ^{2}\left(\Omega_{\mathrm{i}} \Delta t\right),
$$

which reduces to (4.11) for small $\Delta t$ but also falls off quickly enough as $\Delta t \rightarrow \infty$ [because $\csc (\Omega \Delta t)$ falls exponentially for the $\Omega \propto \sqrt{-i}$ of (4.12)]. But there is no difficulty if the reader thinks some alternative choice would have been simpler or more natural.

We placed scare quotes around the " $a \rightarrow 0$ " limit in (4.17) because we should clarify how it coordinates with the $\epsilon \rightarrow 0$ limit of dimensional regularization. The earlier split (4.15) of the $\Delta t$ integration is invalid unless we can set $d=2$ for $\Delta t \geq a$, up to corrections that disappear as $\epsilon \rightarrow 0$. One feature of dimensional regularization is that it converts integer power-law divergences into non-integer power-law divergences. For example, $\int d(\Delta t) / \Delta t$ might become proportional to $\int d(\Delta t) /(\Delta t)^{1-\epsilon}$ or $\int d(\Delta t) /(\Delta t)^{1-2 \epsilon}$. (We will see details 
later.) When integrated over $\int_{a}^{\infty} d(\Delta t)$, the results will correspondingly have non-integer power-law dependence on the lower cut-off $a$, and we will encounter factors like $(a \Omega)^{\epsilon}$, where $\Omega$ is the relevant frequency scale of the problem. We need to be able to expand such a factor in small $\epsilon$ as $1+\epsilon \ln (a \Omega)+\cdots$ in order to recover the $d=2$ result for the $\Delta t \geq a$ integral, which means that $\epsilon \ln (a \Omega)$ will need to be small. The " $a \rightarrow 0$ " limit in (4.17) is therefore notational short-hand for expanding expressions assuming that the small $a$ has been chosen relative to the (eventually) arbitrarily small $\epsilon$ such that

$$
\epsilon \ll \frac{1}{|\ln (a \Omega)|} \rightarrow 0
$$

Before moving on to discuss the application of these techniques to the $x y y \bar{x}$ diagram of figure 29, we should clarify how we plan to handle photon and fermion helicities in dimensional regularization. We will implement the Conventional Dimensional Regularization (CDR) scheme with $\overline{\mathrm{MS}}$ renormalization. These are, for instance, the conventions used by the Particle Data Group [26] and the community at large to quote, compare, and take world-averages of determinations of $\alpha_{\mathrm{S}}\left(M_{\mathrm{Z}}\right)$. For a nicely brief summary of different flavors of "dimensional regularization" used by some authors, see, for example, section II.A of ref. $[25] .^{23}$

\subsubsection{Dimensional regularization: application to $x y y \bar{x}$}

The $\delta H$ matrix element in (4.8), which are the vertex factors of figures 17 and 19 and appendix D, are all proportional to the transverse momentum $\boldsymbol{P}$ characterizing the splitting, which is equal to the $\boldsymbol{P}_{i j}$ of any pair of the particles directly involved in the splitting (or merging). Below, take this to be the $\boldsymbol{P}_{i j}$ of the two daughters. The proportionality of $\delta H$ matrix elements to $\boldsymbol{P}$ holds in any dimension $d$. The proportionality constant, which involves DGLAP splitting amplitudes and consideration of particle helicities, depends on $d$, but for the moment we won't keep track of those details. In transverse-position space, the factors of $\boldsymbol{P}$ become factors of $\pm i \boldsymbol{\nabla} \delta^{(d)}(\boldsymbol{B})$. Plugging this into the starting formula (4.8) for the $x y y \bar{x}$ diagram, and using the $\delta$ functions to perform the implicit integrations over the $\boldsymbol{B}$ variables, gives

$$
\begin{aligned}
{\left[\frac{d \Gamma}{d x_{e} d y_{e}}\right]_{x y y \bar{x}} \propto } & \left.\int_{\text {times }} \nabla_{\boldsymbol{B}^{\overline{\mathrm{x}}}}\left\langle\boldsymbol{B}^{\overline{\mathrm{x}}}, t_{\overline{\mathrm{x}}} \mid \boldsymbol{B}^{\mathrm{y}^{\prime}}, t_{\mathrm{y}^{\prime}}\right\rangle\right|_{\boldsymbol{B}^{\overline{\mathrm{x}}}=0} \\
& \times \nabla_{\boldsymbol{C}_{23}^{\mathrm{y}}},\left.\nabla_{\boldsymbol{C}_{23}^{\mathrm{y}}}\left\langle\boldsymbol{C}_{41}^{\mathrm{y}^{\prime}}, \boldsymbol{C}_{23}^{\mathrm{y}^{\prime}}, t_{\mathrm{y}^{\prime}} \mid \boldsymbol{C}_{41}^{\mathrm{y}}, \boldsymbol{C}_{23}^{\mathrm{y}}, t_{\mathrm{y}}\right\rangle\right|_{\boldsymbol{C}_{23}^{\mathrm{y}^{\prime}}=0=\boldsymbol{C}_{23}^{\mathrm{y}} ; \boldsymbol{C}_{41}^{\mathrm{y}^{\prime}}=\boldsymbol{B}^{\mathrm{y}^{\prime}} ; \boldsymbol{C}_{41}^{\mathrm{y}}=\boldsymbol{B}^{\mathrm{y}}} \\
& \times\left.\nabla_{\boldsymbol{B}^{\mathrm{x}}}\left\langle\boldsymbol{B}^{\mathrm{y}}, t_{\mathrm{y}} \mid \boldsymbol{B}^{\mathrm{x}}, t_{\mathrm{x}}\right\rangle\right|_{\boldsymbol{B}^{\mathrm{x}}=0}
\end{aligned}
$$

\footnotetext{
${ }^{23}$ For the quantities computed in this paper, there will be no difference between using what ref. [25] identifies as CDR and HV dimensional regularization, once we write final formulas for differential rates in terms of MS-bar renormalized $\alpha$ and set $\epsilon=0$. In particular, for the quantities we calculate, we will not have any IR/collinear divergences that need to be canceled between real and virtual emission diagrams, and so it does not matter if we treat real and virtual particles on exactly the same footing in $d=2-\epsilon$ dimensions. In part this is thanks to the fact that medium effects cut off infrared and collinear singularities for splittings contained within the medium.
} 
where the various gradients $\boldsymbol{\nabla}$ are contracted in ways that depend on the helicity-dependent DGLAP splitting functions not explicitly shown here. The integral over the relative vertex times in the $x y y \bar{x}$ time-ordered diagram of figure 29 can be written as

$$
\int_{\text {times }} \cdots=\int_{0}^{\infty} d(\Delta t) \int_{0}^{\infty} d\left(t_{\mathrm{y}}-t_{\mathrm{x}}\right) \int_{0}^{\infty} d\left(t_{\overline{\mathrm{x}}}-t_{\mathrm{y}^{\prime}}\right) \cdots
$$

In the multiple scattering $(\hat{q})$ approximation, the 3-particle (effectively 1-particle) propagators $\left\langle\boldsymbol{B}^{\prime}, t^{\prime} \mid \boldsymbol{B}, t\right\rangle$ are equivalent to those of a single $d$-dimensional harmonic oscillator in non-relativistic quantum mechanics, whose mass $M$ and (complex) frequency $\Omega$ are related to the longitudinal momentum fractions of the three particles. In the particular case of the $x y y \bar{x}$ diagram, the momentum fractions are the same for both regions of 3-particle evolution and give the $\Omega_{\mathrm{i}}$ of (4.12) and

$$
M_{\mathrm{i}}=x_{e}\left(1-x_{e}\right) E .
$$

The formula for a harmonic oscillator propagator,

$$
\left(\frac{M \Omega \csc (\Omega \Delta t)}{2 \pi i}\right)^{d / 2} \exp \left(\frac{i}{2} M \Omega\left[\left(\boldsymbol{B}^{2}+\boldsymbol{B}^{\prime 2}\right) \cot (\Omega \Delta t)-2 \boldsymbol{B} \cdot \boldsymbol{B}^{\prime} \csc (\Omega \Delta t)\right]\right)
$$

is simple enough that the integrals over the initial and final times in (4.20) can be carried out explicitly [9], giving ${ }^{24}$

$$
\begin{aligned}
{\left[\frac{d \Gamma}{d x_{e} d y_{e}}\right]_{x y y \bar{x}} \propto } & \int_{0}^{\infty} d(\Delta t) \int_{\boldsymbol{B}^{\mathrm{y}^{\prime}}, \boldsymbol{B}^{\mathrm{y}}} \frac{\boldsymbol{B}^{\mathrm{y}^{\prime}} \boldsymbol{B}^{\mathrm{y}}}{\left[\left(B^{\mathrm{y}^{\prime}}\right)^{2}\left(B^{\mathrm{y}}\right)^{2}\right]^{d / 4}} \\
& \times K_{d / 4}\left(\frac{1}{2}\left|M_{\mathrm{i}}\right| \Omega_{\mathrm{i}}\left(B^{\mathrm{y}^{\prime}}\right)^{2}\right) K_{d / 4}\left(\frac{1}{2}\left|M_{\mathrm{i}}\right| \Omega_{\mathrm{i}}\left(B^{\mathrm{y}}\right)^{2}\right) \\
& \times \nabla_{\boldsymbol{C}_{23}^{\mathrm{y}}},\left.\nabla_{\boldsymbol{C}_{23}^{\mathrm{y}}}\left\langle\boldsymbol{C}_{41}^{\mathrm{y}}{ }^{\prime}, \boldsymbol{C}_{23}^{\mathrm{y}}{ }^{\prime}, t_{\mathrm{y}}^{\prime} \mid \boldsymbol{C}_{41}^{\mathrm{y}}, \boldsymbol{C}_{23}^{\mathrm{y}}, t_{\mathrm{y}}\right\rangle\right|_{\boldsymbol{C}_{23}^{\mathrm{y}}{ }^{\prime}=0=\boldsymbol{C}_{23}^{\mathrm{y}} ; \boldsymbol{C}_{41}^{\mathrm{y}}{ }^{\prime}=\boldsymbol{B}^{\mathrm{y}^{\prime}} ; \boldsymbol{C}_{41}^{\mathrm{y}}=\boldsymbol{B}^{\mathrm{y}}},
\end{aligned}
$$

where now it is the two $\boldsymbol{B} \mathrm{s}$ and two $\boldsymbol{\nabla}_{\mathrm{s}}$ that are contracted in ways that depend on the helicity-dependent DGLAP splitting functions. The 4-particle (effectively 2-particle) propagator $\left\langle\boldsymbol{C}_{41}^{\prime}, \boldsymbol{C}_{23}^{\prime}, t^{\prime} \mid \boldsymbol{C}_{41}, \boldsymbol{C}_{23}, t\right\rangle$ is also a harmonic oscillator propagator, but this time for a coupled set of two harmonic oscillators. A number of terms are generated when one takes derivatives $\nabla_{C_{23}^{\prime}} \nabla_{C_{23}}$ of this propagator as in (4.24), but only one of them leads to divergences of the $\Delta t$ integral when $\epsilon=0$, and that is then the only term we need to treat with dimensional regularization. Details are given in appendix F. The small- $\Delta t$ behavior

\footnotetext{
${ }^{24}$ The absolute value signs on $\left|M_{\mathrm{i}}\right|$ in (4.24) may at first look redundant, since $M_{\mathrm{i}}$ given by (4.22) is positive for physical values $\left(0<x_{e}<1\right)$ of $x_{e}$. However, we would like to be able to make front-end transformations (4.2) of our results, and that transformation replaces the original $x_{e}$ by negative values (for physical values of the new $x_{e}$ ) and so replaces the $M_{\mathrm{i}}$ of (4.22) by something negative. AI1 section V.A [7] discusses how allowing for negative values of $M_{\mathrm{i}}$ requires introducing absolute value signs in certain places, and the generalization to $d$ dimensions is discussed in ACI3 section IV.A [9].
} 
of that term, which is all we need to regulate to implement (4.17), is specifically

$$
\begin{aligned}
{\left[\frac{d \Gamma}{d x_{e} d y_{e}}\right]_{x y y \bar{x}}^{(\Delta t<a)} \propto } & P_{e \rightarrow e}^{(d)}\left(x_{e}\right) P_{\gamma \rightarrow e}^{(d)}\left(\frac{y_{e}}{1-x_{e}}\right) \int_{0}^{a} \frac{d(\Delta t)}{(\Delta t)^{d+1}} \\
& \times \int_{\boldsymbol{B}^{\mathrm{y}^{\prime}, \boldsymbol{B}^{\mathrm{y}}}} \frac{\boldsymbol{B}^{\mathrm{y}^{\prime}} \cdot \boldsymbol{B}^{\mathrm{y}}}{\left[\left(B^{\mathrm{y}^{\prime}}\right)^{2}\left(B^{\mathrm{y}}\right)^{2}\right]^{d / 4}} K_{d / 4}\left(\frac{1}{2}\left|M_{\mathrm{i}}\right| \Omega_{\mathrm{i}}\left(B^{\mathrm{y}}\right)^{2}\right) K_{d / 4}\left(\frac{1}{2}\left|M_{\mathrm{i}}\right| \Omega_{\mathrm{i}}\left(B^{\mathrm{y}^{\prime}}\right)^{2}\right) \\
& \times \exp \left[-\frac{1}{2} \mathcal{X}_{\mathrm{y}}\left(B^{\mathrm{y}}\right)^{2}-\frac{1}{2} \mathcal{X}_{\mathrm{y}^{\prime}}\left(B^{\mathrm{y} \prime}\right)^{2}+\mathcal{X}_{\mathrm{yy}} \boldsymbol{B}^{\mathrm{y}} \cdot \boldsymbol{B}^{\mathrm{y} \prime}\right]
\end{aligned}
$$

where the exponential factor is analogous to the exponential factor in the single harmonic oscillator propagator (4.23) and corresponds to the exponential of the double harmonic oscillator problem after setting $\boldsymbol{C}_{23}^{\prime}=0=\boldsymbol{C}_{23}$. The small- $\Delta t$ expansions of the coefficients $\mathcal{X}$ in the exponent turn out to be

$$
\begin{aligned}
\mathcal{X}_{\mathrm{y}}=\mathcal{X}_{\mathrm{y}^{\prime}} & =-\frac{i M_{\mathrm{i}}}{\Delta t}+\frac{i M_{\mathrm{i}} \Omega_{\mathrm{i}}^{2} \Delta t}{3}+O\left(M \Omega^{4}(\Delta t)^{3}\right), \\
\mathcal{X}_{\mathrm{yy}^{\prime}} & =-\frac{i M_{\mathrm{i}}}{\Delta t}-\frac{i M_{\mathrm{i}} \Omega_{\mathrm{i}}^{2} \Delta t}{6}+O\left(M \Omega^{4}(\Delta t)^{3}\right) .
\end{aligned}
$$

[The fact that the expansions to this order can be written in terms of $\Omega_{\mathrm{i}}$ (4.12), without reference to the actual eigenfrequencies $\Omega_{ \pm}$of the double harmonic oscillator problem, is non-trivial. See appendix F.2.2.]

In (4.25), we also now show explicitly the particular combination of DGLAP splitting functions that appear, which for this divergent term happen to combine into a simple product of the $(d=2-\epsilon$ dimensional $)$ spin-averaged splitting functions $P_{e \rightarrow e}^{(d)}$ and $P_{\gamma \rightarrow e}^{(d)}$ for $e \rightarrow \gamma e$ and $\gamma \rightarrow e \bar{e}$.

The technical problem we face to carry out dimensional regularization is how to do the integrals in (4.25) with (4.26) in the small-a limit (4.19). For similar integrals in ref. [9] (ACI3), we were able to argue that the exponential factor in the integrand limited the range of the $B \mathrm{~s}$ enough in the small- $\Delta t$ limit that one could make small-argument expansions of the Bessel functions $K_{\mathrm{d} / 4}\left(\frac{1}{2}\left|M_{\mathrm{i}}\right| \Omega_{\mathrm{i}} B^{2}\right)$. Unfortunately, that is not the case here. If the exponent in (4.25) were evaluated with (4.26) at leading order in small $\Delta t$, the exponential would be

$$
\exp \left[\frac{i M_{\mathrm{i}}}{2 \Delta t}\left(\boldsymbol{B}^{\mathrm{y} \prime}-\boldsymbol{B}^{\mathrm{y}}\right)^{2}\right]
$$

This oscillating exponential will suppress contributions to the integral unless $\left|\boldsymbol{B}^{\mathrm{y}}-\boldsymbol{B}^{\mathrm{y}}\right| \lesssim$ $\Delta t / M_{\mathrm{i}}$, and so

$$
\left|\boldsymbol{B}^{\mathrm{y} \prime}-\boldsymbol{B}^{\mathrm{y}}\right| \lesssim \frac{a}{M_{\mathrm{i}}}
$$

since $\Delta t \leq a$ in (4.25). But (4.27) places no constraint on how large $\boldsymbol{B}^{\mathrm{y} \prime} \simeq \boldsymbol{B}^{\mathrm{y}}$ can be. That is cut off instead by the exponential fall-off of the Bessel functions in (4.25) for large arguments. [The sub-leading terms of (4.26) are not large enough to cut off the integrand sooner.] The Bessel functions suppress contributions unless

$$
B \lesssim\left|M_{\mathrm{i}} \Omega_{\mathrm{i}}\right|^{-1 / 2}
$$


The integrand remains unsuppressed when the arguments of the Bessel function are of order 1, and so small-argument expansions of the Bessel functions are not applicable. Having to deal with the Bessel functions as they are makes the integral harder to do.

In appendix $\mathrm{G}$, we show how to evaluate the integral

$$
\begin{gathered}
\mathbb{I} \equiv \frac{i M(|M| \Omega)^{d / 2}}{\pi} \int_{0}^{a} \frac{d(\Delta t)}{(\Delta t)^{d+1}} \int_{\boldsymbol{B}, \boldsymbol{B}^{\prime}} \frac{\boldsymbol{B} \cdot \boldsymbol{B}^{\prime}}{\left(B^{2}\right)^{d / 4}\left(B^{\prime 2}\right)^{d / 4}} K_{d / 4}\left(\frac{1}{2}|M| \Omega B^{2}\right) K_{d / 4}\left(\frac{1}{2}|M| \Omega B^{\prime 2}\right) \\
\times \exp \left[-\frac{1}{2} \mathcal{X}_{\mathrm{y}} B^{2}-\frac{1}{2} \mathcal{X}_{\mathrm{y}^{\prime}}{B^{\prime 2}}^{2} \mathcal{X}_{\mathrm{yy}} \boldsymbol{B} \cdot \boldsymbol{B}^{\prime}\right]
\end{gathered}
$$

which is proportional to the one shown in (4.25), to obtain

$$
\mathbb{I}=2 \pi^{2}(i \bar{\Omega})^{d-1}\left[-\left(\frac{2}{\epsilon}-\gamma_{\mathrm{E}}+\ln (4 \pi)\right)-\frac{\ln (2 i \bar{\Omega} a)+1}{i \bar{\Omega} a}-\ln (i \bar{\Omega} a)+3 \ln (2 \pi)\right]+O(a)+O(\epsilon),
$$

where $\gamma_{\mathrm{E}}$ is Euler's constant and ${ }^{25}$

$$
\bar{\Omega} \equiv \Omega \operatorname{sgn}(M)
$$

The $a$ dependence will be canceled, as it must, when one adds together all the terms of (4.17) for the calculation of $x y y \bar{x}$. When we later combine the $x y y \bar{x}$ diagram with leading-order $e \rightarrow \gamma e$, the $1 / \epsilon$ pole will be absorbed, as it must, by the known renormalization of $\alpha_{\mathrm{EM}}$ in QED.

The rest of the calculation of $x y y \bar{x}$ is mostly just a matter of combining the above with calculations and formulas that are closely analogous to those for diagrams analyzed in our previous work [7-9]. We will write our decomposition (4.17) of the calculation as

$$
2 \operatorname{Re}\left[\frac{d \Gamma}{d x_{e}}\right]_{x y y \bar{x}}=\lim _{a \rightarrow 0 "}\left\{2 \operatorname{Re}\left[\frac{d \Gamma}{d x_{e}}\right]_{x y y \bar{x}}^{(\Delta t<a)}+2 \operatorname{Re}\left[\frac{d \Gamma}{d x_{e}}\right]_{x y y \bar{x}}^{\left(\mathcal{D}_{2}\right)}\right\}+2 \operatorname{Re}\left[\frac{d \Gamma}{d x_{e}}\right]_{x y y \bar{x}}^{\text {(subtracted) }}
$$

where $\left[d \Gamma / d x_{e}\right]^{\text {(subtracted) }}$ represents the $\int d(\Delta t)\left[F_{2}(\Delta t)-\mathcal{D}_{2}(\Delta t)\right]$ term in (4.17). Details are given in appendix F.

\subsubsection{Renormalization}

There are two equivalent ways to think about renormalization and the ultimate cancellation of the $x y y \bar{x}$ UV divergence appearing in (4.31). One is renormalized perturbation theory: add counter-term diagrams such as figure 30 , so that the combination

$$
\left[\frac{d \Gamma}{d x_{e}}\right]_{\mathrm{xyy} \overline{\mathrm{x}}}+\left[\frac{d \Gamma}{d x_{e}}\right]_{\substack{\text { counterterm } \\ \text { diagram }}}
$$

is finite. Note that in the large- $N_{\mathrm{f}}$ limit of $\mathrm{QED}$, there is no electron wave function renormalization and no vertex renormalization, and in QED the photon wave function renormalization is equivalent to charge renormalization.

\footnotetext{
${ }^{25}$ The notation $\bar{\Omega}$ defined by (4.32) is unrelated to the notation $\bar{\Omega}_{\mathrm{f}}$ in AI1 section VI.B [7]: there are only so many accent marks, and we've been forced to recycle.
} 

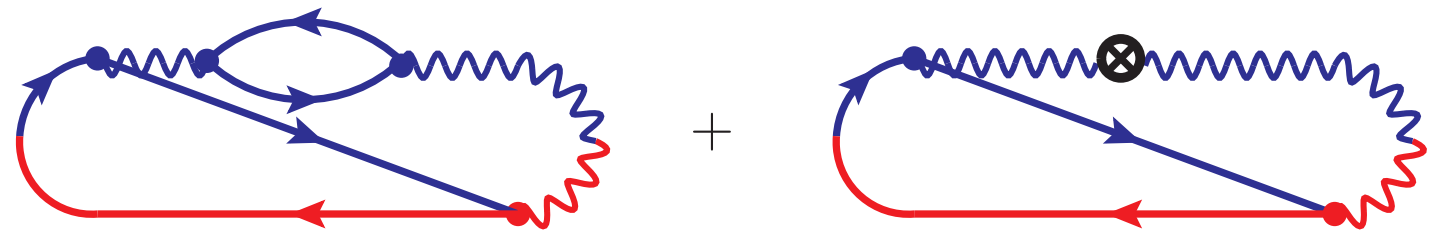

Figure 30. Renormalization of the $x y y \bar{x}$ diagram by adding the corresponding (time-ordered) counter-term diagram.

The other viewpoint, which we find more straightforward to implement,${ }^{26}$ is to imagine that the contribution to $d \Gamma / d x_{e}$ from all $e \rightarrow \gamma e$ diagrams, including the leading-order process represented by the $x \bar{x}$ diagram of figure 31 (plus its complex conjugate), are initially computed using the bare coupling instead of the renormalized coupling. Afterwards, we convert to the $\overline{\mathrm{MS}}$-renormalized coupling using the known relation

$$
\alpha_{\text {bare }}=\alpha_{\text {ren }}+\frac{N_{\mathrm{f}} \alpha_{\text {ren }}^{2}}{3 \pi}\left(\frac{2}{\epsilon}-\gamma_{\mathrm{E}}+\ln (4 \pi)\right)+O\left(\alpha^{3}\right) \quad(\mathrm{QED}) .
$$

When expressed in terms of renormalized $\alpha$, the $1 / \epsilon$ divergences will then cancel in the combination

$$
\left[\frac{d \Gamma}{d x_{e}}\right]_{\mathrm{x} \overline{\mathrm{x}}}+\left[\frac{d \Gamma}{d x_{e}}\right]_{\mathrm{xyy} \overline{\mathrm{x}}}
$$

through order $\alpha^{2}$. Since the leading order $\left[d \Gamma / d x_{e}\right]_{\mathrm{x} \overline{\mathrm{x}}}^{\mathrm{bare}}$ is proportional to $\alpha_{\mathrm{bare}},(4.35)$ gives

$$
\left[\frac{d \Gamma}{d x_{e}}\right]_{\mathrm{x} \overline{\mathrm{x}}}^{(\text {bare })}=\left[\frac{d \Gamma}{d x_{e}}\right]_{\mathrm{x} \overline{\mathrm{x}}}^{(\mathrm{ren})}+\frac{N_{\mathrm{f}} \alpha_{\mathrm{ren}}}{3 \pi}\left[\frac{d \Gamma}{d x_{e}}\right]_{\mathrm{x} \overline{\mathrm{x}}}^{(\mathrm{ren})}\left(\frac{2}{\epsilon}-\gamma_{\mathrm{E}}+\ln (4 \pi)\right)+O\left(\alpha^{3}\right) .
$$

We will combine the second term on the right-hand side with $x y y \bar{x}$ to define

$$
\left[\frac{d \Gamma}{d x_{e}}\right]_{\mathrm{xyy} \overline{\mathrm{x}}}^{(\mathrm{ren})}=\left[\frac{d \Gamma}{d x_{e}}\right]_{\mathrm{xyy} \overline{\mathrm{x}}}+\frac{N_{\mathrm{f}} \alpha_{\mathrm{ren}}}{3 \pi}\left[\frac{d \Gamma}{d x_{e}}\right]_{\mathrm{x} \overline{\mathrm{x}}}^{(\mathrm{ren})}\left(\frac{2}{\epsilon}-\gamma_{\mathrm{E}}+\ln (4 \pi)\right),
$$

which is equivalent to figure 30 . Note that, because it is multiplied by $2 / \epsilon$, we will need to use a $d=2-\epsilon$ formula for the leading-order $\left[d \Gamma / d x_{e}\right]_{\mathrm{x} \overline{\mathrm{x}}}$ above, expanded through $O(\epsilon)$. The explicit formula is given in (F.44) of appendix F.

\footnotetext{
${ }^{26}$ The second viewpoint avoids having to sort out a few possible sources of confusion. For example, when computing amplitudes in quantum field theory, one multiplies external legs by $Z_{\text {field }}^{1 / 2}$ instead of $Z_{\text {field }}$, but it is the latter that comes from the divergences of self-energy loops such as the photon self-energy loop in the $x y y \bar{x}$ diagram. The seeming difference can be resolved by realizing that the leading-order $x \bar{x}$ diagram represents a rate rather than an amplitude, and so should be multiplied by $\left|Z_{\text {field }}^{1 / 2}\right|^{2}=Z_{\text {field }}$, which generates the counter-term diagram in figure 30. But one could also worry about why we have not included other time-orderings of that counter-term diagram, such as ones where the counter-term occurs later than both emission vertices. Those contributions turn out to cancel between diagrams with the counter-term in the amplitude and diagrams with the counter-term in the conjugate amplitude, because of the different signs in the corresponding evolution operators $\exp (\mp i \delta H t)$. The second viewpoint on renormalization allows us to bypass all of these considerations.
} 


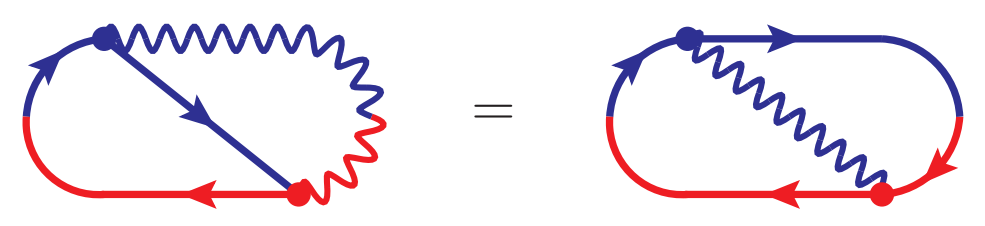

Figure 31. (left) Leading-order time-ordered diagram $x \bar{x}$ for $e \rightarrow \gamma e$. This diagram should be added to its complex conjugate by taking $2 \operatorname{Re}[\cdots]$. (right) A completely equivalent way to draw this diagram.

Now combine the decomposition (4.33) of $x y y \bar{x}$ into calculable divergent and finite parts with the renormalization (4.38), and choose $\mathcal{D}_{2}(\Delta t)$ according to (4.18). We find (see appendix F.6)

$$
\left[\frac{d \Gamma}{d x_{e}}\right]_{x y y \bar{x}}^{(\mathrm{ren})}=-\frac{N_{\mathrm{f}} \alpha_{\mathrm{EM}}}{3 \pi}\left[\frac{d \Gamma}{d x_{e}}\right]_{x \bar{x}}\left(\ln \left(\frac{\mu^{2}}{\left(1-x_{e}\right) E \Omega_{\mathrm{i}}}\right)+\gamma_{\mathrm{E}}-2 \ln 2+\frac{5}{3}\right)+\left[\frac{d \Gamma}{d x_{e}}\right]_{x y y \bar{x}}^{\text {(subtracted) }},
$$

where $\mu$ is the renormalization scale. $\left[d \Gamma / d x_{e}\right]^{\text {(subtracted) }}$ is as defined earlier in (4.33), with the result given by (F.40) of appendix F.

An aspect of handling $x y y \bar{x}$ divergences worth mentioning here, that was unnecessary in our previous application of dimensional regularization to real double splitting $g \rightarrow g g g$ in ref. [9] (ACI3), is that we find we need the $d=2-\epsilon$ result for a DGLAP splitting function - specifically $P_{\gamma \rightarrow e \bar{e}}^{(d)}$, which is the additional splitting function factor in the $x y y \bar{x}$ divergence (4.25) compared to the leading-order $x \bar{x}$ diagram of figure 31.

\subsection{Relation to the $\gamma \rightarrow e \bar{e}$ virtual diagram (r)}

As mentioned earlier in section 4.2, the diagram of figure 9r for NLO $\gamma \rightarrow e \bar{e}$ is related to the diagram of figure 8k just discussed by a combination of front-end and back-end transformations. Specifically, similar to (4.4),

$$
2 \operatorname{Re}\left[\frac{d \Gamma}{d y_{e}}\right]_{(\mathrm{r})}=+2 N_{\mathrm{f}} \operatorname{Re} \int_{0}^{1} d x_{e}\left\{\left[\frac{d \Gamma}{d x_{e} d y_{e}}\right]_{(\mathrm{k})} \quad \begin{array}{l}
\text { with substitutions }(4.2) \\
\text { followed by } x_{e} \leftrightarrow y_{e}
\end{array}\right\},
$$

where $\left[d \Gamma / d x_{e} d y_{e}\right]_{(\mathrm{k})}$ represents the $y_{e}$-integrand that integrates to (4.39), which may be interpreted as

$$
\begin{aligned}
{\left[\frac{d \Gamma}{d x_{e} d y_{e}}\right]_{x y y \bar{x}}^{(\mathrm{ren})} } & -\frac{N_{\mathrm{f}} \alpha_{\mathrm{EM}}}{3 \pi}\left[\frac{d \Gamma}{d x_{e}}\right]_{x \bar{x}}\left(\ln \left(\frac{\mu^{2}}{\left(1-x_{e}\right) E \bar{\Omega}_{\mathrm{i}}}\right)+\gamma_{\mathrm{E}}-2 \ln 2+\frac{5}{3}\right) \delta\left(y_{e}\right) \\
& +\left[\frac{d \Gamma}{d x_{e} d y_{e}}\right]_{x y y \bar{x}}^{(\text {subtracted })}
\end{aligned}
$$

with the understanding that $\int_{0}^{1} d y_{e} \delta\left(y_{e}\right)=1$. Above, the use of $\bar{\Omega}_{\mathrm{i}} \equiv \Omega_{\mathrm{i}} \operatorname{sgn}\left(M_{\mathrm{i}}\right)$ is necessary to generalize the $x y y \bar{x}$ result to work with front-end transformations, as explained in appendix F.2.3. 
Since we combine leading and next-to-leading order results when we renormalize as in (4.37), we note here that the relation between leading-order results for $\gamma \rightarrow e \bar{e}$ and $e \rightarrow \gamma e$ is ${ }^{27}$

$$
2 \operatorname{Re}\left[\frac{d \Gamma}{d y_{e}}\right]_{y \bar{y}}^{\gamma \rightarrow e \bar{e}}=2 N_{\mathrm{f}}\left(1-y_{e}\right) \operatorname{Re}\left\{\left[\frac{d \Gamma}{d x_{e}}\right]_{x \bar{x}}^{e \rightarrow \gamma e} \quad \begin{array}{l}
\text { with substitutions (4.2) } \\
\text { followed by } x_{e} \leftrightarrow y_{e}
\end{array}\right\} .
$$

This can be understood as an application of the same transformation law (4.40) if one interprets $\left[d \Gamma / d x_{e} d y_{e}\right]_{x \bar{x}}^{e \rightarrow \gamma e} \equiv\left[d \Gamma / d x_{e}\right]_{x \bar{x}}^{e \rightarrow \gamma e} \delta\left(y_{e}\right)$.

\section{Conclusion}

A complete summary of our final formulas for rates is given in appendix A. The work in this paper completes the analytic calculation of the effect of overlapping formation times in QED in the simplifying parametric limit $\alpha \ll N_{\mathrm{f}} \alpha \ll 1$. Even though that is a QED limit only a theorist could love, we think it will be interesting to look at the size of those effects on shower development as one changes the value of $N_{\mathrm{f}} \alpha$ to be closer and closer to $O(1)$, as a first exploration of how to carry out similar calculations for QCD if one pushes $N_{\mathrm{c}} \alpha_{\mathrm{s}}\left(Q_{\perp}\right)$ to be larger and larger to answer the question of how small or overwhelming overlap effects are in practice. We leave that QED analysis, as well as discussion of numerical results for the formulas presented here, to forthcoming work [16].

With these QED results in hand, it should now be possible to complete similar calculations for large- $N_{\mathrm{c}} \mathrm{QCD}$.

\section{Acknowledgments}

We are quite grateful to Diana Vaman, Yuri Kovchegov, Risto Paatelainen, Tuomas Lappi, Guillaume Beuf, Aleksi Kurkela, John Collins, R. Keith Ellis, and Zvi Bern for useful conversations. This work was supported, in part, by the U.S. Department of Energy under Grant No. DE-SC0007984.

\section{A Summary of results}

In this appendix, we gather our results for three different quantities:

$$
\left[\frac{\Delta d \Gamma}{d x_{e} d y_{e}}\right]_{e \rightarrow e \bar{e} e}, \quad\left[\frac{d \Gamma}{d x_{e}}\right]_{e \rightarrow \gamma e}, \quad \text { and } \quad\left[\frac{d \Gamma}{d y_{e}}\right]_{\gamma \rightarrow e \bar{e}} .
$$

The first is the total overlap correction of figure 7 to real double splitting $e \rightarrow \gamma e \rightarrow e \bar{e} e$. The last two are the single splitting rates expanded through next-to-leading order,

$$
\begin{aligned}
& {\left[\frac{d \Gamma}{d x_{e}}\right]_{e \rightarrow \gamma e}=\left[\frac{d \Gamma}{d x_{e}}\right]_{e \rightarrow \gamma e}^{\mathrm{LO}}+\left[\frac{\Delta d \Gamma}{d x_{e}}\right]_{e \rightarrow \gamma e}^{\mathrm{NLO}},} \\
& {\left[\frac{d \Gamma}{d y_{e}}\right]_{\gamma \rightarrow e \bar{e}}=\left[\frac{d \Gamma}{d y_{e}}\right]_{\gamma \rightarrow e \bar{e}}^{\mathrm{LO}}+\left[\frac{\Delta d \Gamma}{d y_{e}}\right]_{\gamma \rightarrow e \bar{e}}^{\mathrm{NLO}},}
\end{aligned}
$$

\footnotetext{
${ }^{27}$ The normalization factor $\left(1-y_{e}\right)$ in $(4.42)$ is the type of normalization mentioned in footnote 21 . For the overall sign to work in (4.42), one must use the generalized formula (F.47) for $\left[d \Gamma / d x_{e}\right]_{x \bar{x}}$ that works for any sign of $M_{\mathrm{i}}$.
} 
where the NLO contributions are given by figures 8 and 9 respectively. Note that our convention is to use $x_{e}$ to refer to the final momentum fraction of an electron after bremsstrahlung and $y_{e}$ to refer to the final momentum fraction of the electron in an $e \bar{e}$ pair that was pair produced.

\section{A.1 Leading-order single-splitting rates}

As discussed in appendix C, the leading-order results for single splitting in QED go all the way back to Migdal [3].

\section{A.1.1 $e \rightarrow \gamma e$}

In our notation, the leading-order $e \rightarrow \gamma e$ rate is ${ }^{28}$

$$
\left[\frac{d \Gamma}{d x_{e}}\right]_{e \rightarrow \gamma e}^{\mathrm{LO}}=\frac{\alpha}{\pi} P_{e \rightarrow e}\left(x_{e}\right) \operatorname{Re}\left(i \Omega_{0}\right)=\frac{\alpha}{\pi} P_{e \rightarrow \gamma}\left(x_{\gamma}\right) \operatorname{Re}\left(i \Omega_{0}\right)
$$

with $x_{\gamma}=1-x_{e}$,

$$
\Omega_{0}=\sqrt{\frac{-i\left(1-x_{e}\right) \hat{q}}{2 x_{e} E}}=\sqrt{\frac{-i x_{\gamma} \hat{q}}{2\left(1-x_{\gamma}\right) E}}
$$

and the relevant DGLAP splitting function

$$
P_{e \rightarrow e}\left(x_{e}\right)=P_{e \rightarrow \gamma}\left(x_{\gamma}\right)=\frac{1+x_{e}^{2}}{1-x_{e}}=\frac{1+\left(1-x_{\gamma}\right)^{2}}{x_{\gamma}} .
$$

For the sake of later formulas for virtual corrections, it will later be helpful to also express the above result in terms of the $x \bar{x}$ diagram of figure 31 as

$$
\left[\frac{d \Gamma}{d x_{e}}\right]_{e \rightarrow \gamma e}^{\mathrm{LO}}=2 \operatorname{Re}\left[\frac{d \Gamma}{d x_{e}}\right]_{x \bar{x}} .
$$

\section{A.1.2 $\gamma \rightarrow e \bar{e}$}

Leading-order pair production $\gamma \rightarrow e \bar{e}$ is essentially the same except that

$$
\left[\frac{d \Gamma}{d y_{e}}\right]_{\gamma \rightarrow e \bar{e}}^{\mathrm{LO}}=\frac{N_{\mathrm{f}} \alpha}{\pi} P_{\gamma \rightarrow e}\left(y_{e}\right) \operatorname{Re}\left(i \Omega_{0}^{\gamma \rightarrow e \bar{e}}\right)
$$

with $^{29}$

$$
\Omega_{0}^{\gamma \rightarrow e \bar{e}}=\sqrt{\frac{-i \hat{q}}{2 y_{e}\left(1-y_{e}\right) E}}
$$

and

$$
P_{\gamma \rightarrow e}\left(y_{e}\right)=y_{e}^{2}+\left(1-y_{e}\right)^{2} .
$$

\footnotetext{
${ }^{28}$ QCD versions go back to BDMPS [27-29] and Zakharov [20,21]. To relate the QED and QCD results in our own notation: (A.4) is AI (1.5a) with $P_{g \rightarrow g}(x)$ replaced by $P_{e \rightarrow e}\left(x_{e}\right)$. The formulas for $\Omega_{0}$ in QED and QCD are both special cases of the general formula $\Omega^{2}=-i\left[\left(\hat{q}_{1} / x_{1}\right)+\left(\hat{q}_{2} / x_{2}\right)+\left(\hat{q}_{3} / x_{3}\right)\right] /(2 E)$ of AI (2.33b), where $\hat{q}_{i}$ is zero for the photon.

${ }^{29}$ See footnote 28 .
} 


\section{A.2 Overlap corrections to real double splitting}

We decompose our total results for the overlap correction to real double splitting as

$$
\left[\frac{\Delta d \Gamma}{d x_{e} d y_{e}}\right]_{e \rightarrow e \bar{e} e}=\left[\frac{\Delta d \Gamma}{d x_{e} d y_{e}}\right]_{\mathrm{seq}}+\left[\frac{d \Gamma}{d x_{e} d y_{e}}\right]_{(I)}+\left[\frac{d \Gamma}{d x_{e} d y_{e}}\right]_{(I I)},
$$

where the pieces are given below and " $I$ " indicates a contribution involving an instantaneous 4 -fermion interaction. Below we mostly just collect formulas. See appendix E for a discussion of where those formulas come from.

\section{A.2.1 Sequential diagrams}

The "seq" piece above is our result for diagrams $(\mathrm{a}-\mathrm{c})$ of figure 7 , which are of a type we refer to as "sequential" diagrams in earlier papers. Adopting notation similar to ACI4 appendix D.3 [10], our result is

$$
\left[\frac{\Delta d \Gamma}{d x_{e} d y_{e}}\right]_{\mathrm{seq}}=2 N_{\mathrm{f}} \mathcal{A}_{\mathrm{seq}}\left(x_{e}, y_{e}\right)
$$

with

$$
\begin{aligned}
& \mathcal{A}_{\mathrm{seq}}\left(x_{e}, y_{e}\right) \equiv \mathcal{A}_{\mathrm{seq}}^{\mathrm{pole}}\left(x_{e}, y_{e}\right)+\int_{0}^{+\infty} d(\Delta t)\left[2 \operatorname{Re}\left(B_{\mathrm{seq}}\left(x_{e}, y_{e}, \Delta t\right)\right)+F_{\mathrm{seq}}\left(x_{e}, y_{e}, \Delta t\right)\right] \\
& B_{\mathrm{seq}}\left(x_{e}, y_{e}, \Delta t\right)=C_{\mathrm{seq}}\left(\hat{x}_{1}, \hat{x}_{2}, \hat{x}_{3}, \hat{x}_{4}, \bar{\alpha}, \bar{\beta}, \bar{\gamma}, \Delta t\right) \\
& =C_{\mathrm{seq}}(-1, y, 1-x-y, x, \bar{\alpha}, \bar{\beta}, \bar{\gamma}, \Delta t), \\
& C_{\text {seq }}=D_{\text {seq }}-\lim _{\hat{q} \rightarrow 0} D_{\text {seq }}, \\
& D_{\text {seq }}\left(x_{1}, x_{2}, x_{3}, x_{4}, \bar{\alpha}, \bar{\beta}, \bar{\gamma}, \Delta t\right)= \\
& \frac{\alpha_{\mathrm{EM}}^{2} M_{\mathrm{i}} M_{\mathrm{f}}^{\mathrm{seq}}}{32 \pi^{4} E^{2}}\left(-x_{1} x_{2} x_{3} x_{4}\right) \frac{\Omega_{+} \csc \left(\Omega_{+} \Delta t\right)}{\Delta t} \\
& \times\left\{\left(\bar{\beta} Y_{\mathrm{y}}^{\mathrm{seq}} Y_{\overline{\mathrm{x}}}^{\mathrm{seq}}+\bar{\alpha} \bar{Y}_{\mathrm{y} \overline{\mathrm{x}}}^{\mathrm{seq}} Y_{\mathrm{y} \overline{\mathrm{x}}}^{\mathrm{seq}}\right) I_{0}^{\mathrm{seq}}+(\bar{\alpha}+\bar{\beta}+2 \bar{\gamma}) Z_{\mathrm{y} \overline{\mathrm{x}}}^{\mathrm{seq}} I_{1}^{\mathrm{seq}}\right. \\
& +\left[(\bar{\alpha}+\bar{\gamma}) Y_{\mathrm{y}}^{\mathrm{seq}} Y_{\overline{\mathrm{x}}}^{\mathrm{seq}}+(\bar{\beta}+\bar{\gamma}) \bar{Y}_{\mathrm{y} \overline{\mathrm{x}}}^{\mathrm{seq}} Y_{\mathrm{y} \overline{\mathrm{x}}}^{\mathrm{seq}}\right] I_{2}^{\mathrm{seq}} \\
& \left.-(\bar{\alpha}+\bar{\beta}+\bar{\gamma})\left(\bar{Y}_{\mathrm{y} \overline{\mathrm{x}}}^{\mathrm{seq}} Y_{\overline{\mathrm{x}}}^{\mathrm{seq}} I_{3}^{\mathrm{seq}}+Y_{\mathrm{y}}^{\mathrm{seq}} Y_{\mathrm{y} \overline{\mathrm{x}}}^{\mathrm{seq}} I_{4}^{\mathrm{seq}}\right)\right\}, \\
& F_{\mathrm{seq}}\left(x_{e}, y_{e}, \Delta t\right)=\frac{\alpha_{\mathrm{EM}}^{2} P_{e \rightarrow e}\left(x_{e}\right) P_{\gamma \rightarrow e}\left(\frac{y_{e}}{1-x_{e}}\right)}{4 \pi^{2}\left(1-x_{e}\right)}\left[\operatorname{Re}\left(i \Omega_{\mathrm{i}}\right) \operatorname{Re}\left(\Delta t\left(\Omega_{\mathrm{f}}^{\mathrm{seq}}\right)^{2} \csc ^{2}\left(\Omega_{\mathrm{f}}^{\mathrm{seq}} \Delta t\right)\right)\right. \\
& \left.+\operatorname{Re}\left(i \Omega_{\mathrm{f}}^{\mathrm{seq}}\right) \operatorname{Re}\left(\Delta t \Omega_{\mathrm{i}}^{2} \csc ^{2}\left(\Omega_{\mathrm{i}} \Delta t\right)\right)\right], \\
& \mathcal{A}_{\mathrm{seq}}^{\mathrm{pole}}(x, y)=\frac{\alpha_{\mathrm{EM}}^{2} P_{e \rightarrow e}\left(x_{e}\right) P_{\gamma \rightarrow e}\left(\frac{y_{e}}{1-x_{e}}\right)}{2 \pi^{2}\left(1-x_{e}\right)}\left(-\frac{1}{2} \operatorname{Re}\left(i \Omega_{\mathrm{i}}+i \Omega_{\mathrm{f}}^{\mathrm{seq}}\right)+\frac{\pi}{4} \operatorname{Re}\left(\Omega_{\mathrm{i}}+\Omega_{\mathrm{f}}^{\mathrm{seq}}\right)\right)
\end{aligned}
$$

and

$$
M_{\mathrm{i}}=x_{1} x_{4}\left(x_{1}+x_{4}\right) E, \quad M_{\mathrm{f}}^{\mathrm{seq}}=x_{2} x_{3}\left(x_{2}+x_{3}\right) E .
$$


The $I_{n}^{\text {seq }}$ above represent

$$
\begin{aligned}
I_{0}^{\mathrm{seq}} & =\frac{4 \pi^{2}}{\left[X_{\mathrm{y}}^{\mathrm{seq}} X_{\overline{\mathrm{x}}}^{\mathrm{seq}}-\left(X_{\mathrm{y} \overline{\mathrm{x}}}^{\mathrm{seq}}\right)^{2}\right]}, \\
I_{1}^{\mathrm{seq}} & =-\frac{2 \pi^{2}}{X_{\mathrm{y} \overline{\mathrm{x}}}^{\mathrm{seq}}} \ln \left(1-\frac{\left(X_{\mathrm{y}}^{\mathrm{seq}}\right)^{2}}{X_{\mathrm{y}}^{\mathrm{seq}} X_{\overline{\mathrm{x}}}^{\mathrm{seq}}}\right) \\
I_{2}^{\mathrm{seq}} & =\frac{2 \pi^{2}}{\left(X_{\mathrm{y} \overline{\mathrm{x}}}^{\mathrm{seq}}\right)^{2}} \ln \left(1-\frac{\left(X_{\mathrm{y} \overline{\mathrm{x}}}^{\mathrm{seq}}\right)^{2}}{X_{\mathrm{y}}^{\mathrm{seq}} X_{\overline{\mathrm{x}}}^{\mathrm{seq}}}\right)+\frac{4 \pi^{2}}{\left[X_{\mathrm{y}}^{\mathrm{seq}} X_{\overline{\mathrm{x}}}^{\mathrm{seq}}-\left(X_{\mathrm{y} \overline{\mathrm{x}}}^{\mathrm{seq}}\right)^{2}\right]}, \\
I_{3}^{\mathrm{seq}} & =\frac{4 \pi^{2} X_{\mathrm{y} \overline{\mathrm{x}}}^{\mathrm{seq}}}{X_{\overline{\mathrm{x}}}^{\mathrm{seq}}\left[X_{\mathrm{y}}^{\mathrm{seq}} X_{\overline{\mathrm{x}}}^{\mathrm{seq}}-\left(X_{\mathrm{y} \overline{\mathrm{x}}}^{\mathrm{seq}}\right)^{2}\right]}, \\
I_{4}^{\mathrm{seq}} & =\frac{4 \pi^{2} X_{\mathrm{y} \overline{\mathrm{x}}}^{\mathrm{seq}}}{X_{\mathrm{y}}^{\mathrm{seq}}\left[X_{\mathrm{y}}^{\mathrm{seq}} X_{\overline{\mathrm{x}}}^{\mathrm{seq}}-\left(X_{\mathrm{y} \overline{\mathrm{x}}}^{\mathrm{seq}}\right)^{2}\right]} .
\end{aligned}
$$

Here and in (A.16), the $(X, Y, Z)^{\text {seq }}$ are defined by

$$
\begin{aligned}
& \left(\begin{array}{ll}
X_{\mathrm{y}}^{\mathrm{seq}} & Y_{\mathrm{y}}^{\mathrm{seq}} \\
Y_{\mathrm{y}}^{\mathrm{seq}} & Z_{\mathrm{y}}^{\mathrm{seq}}
\end{array}\right) \equiv\left(\begin{array}{cc}
\left|M_{\mathrm{i}}\right| \Omega_{\mathrm{i}} & 0 \\
0 & 0
\end{array}\right)-i a_{\mathrm{y}}^{-1 \top}\left(\begin{array}{cc}
\Omega_{+} \cot \left(\Omega_{+} \Delta t\right) & 0 \\
0 & (\Delta t)^{-1}
\end{array}\right) a_{\mathrm{y}}^{-1}, \\
& \left(\begin{array}{cc}
X_{\overline{\mathrm{x}}}^{\mathrm{seq}} & Y_{\overline{\mathrm{x}}}^{\mathrm{seq}} \\
Y_{\overline{\mathrm{x}}}^{\mathrm{seq}} & Z_{\overline{\mathrm{x}}}^{\mathrm{seq}}
\end{array}\right) \equiv\left(\begin{array}{cc}
\left|M_{\mathrm{f}}^{\mathrm{seq}}\right| \Omega_{\mathrm{f}}^{\mathrm{seq}} & 0 \\
0 & 0
\end{array}\right)-i\left(a_{\overline{\mathrm{x}}}^{\mathrm{seq}}\right)^{-1 \top}\left(\begin{array}{cc}
\Omega_{+} \cot \left(\Omega_{+} \Delta t\right) & 0 \\
0 & (\Delta t)^{-1}
\end{array}\right)\left(a_{\overline{\mathrm{x}}}^{\mathrm{seq}}\right)^{-1}, \\
& \left(\begin{array}{ll}
X_{\mathrm{y}}^{\mathrm{seq}} & Y_{\mathrm{y} \overline{\mathrm{x}}}^{\mathrm{seq}} \\
\bar{Y}_{\mathrm{y} \overline{\mathrm{x}}}^{\mathrm{seq}} & Z_{\mathrm{y} \overline{\mathrm{x}}}^{\mathrm{seq}}
\end{array}\right) \equiv-i a_{\mathrm{y}}^{-1 \top}\left(\begin{array}{cc}
\Omega_{+} \csc \left(\Omega_{+} \Delta t\right) & 0 \\
0 & (\Delta t)^{-1}
\end{array}\right)\left(a_{\overline{\mathrm{x}}}^{\mathrm{seq}}\right)^{-1},
\end{aligned}
$$

where the $a$ 's and $\Omega$ 's will be given below. The quantities $(\bar{\alpha}, \bar{\beta}, \bar{\gamma})$ in (A.14) represent various combinations of helicity-dependent DGLAP splitting functions and are

$$
\left(\begin{array}{c}
\bar{\alpha} \\
\bar{\beta} \\
\bar{\gamma}
\end{array}\right)_{e \rightarrow e \bar{e} e}=\frac{1}{\left(1-x_{e}\right)^{6}}\left\{\left(\begin{array}{c}
- \\
+ \\
+
\end{array}\right) \frac{4}{\left|x_{e} y_{e} z_{e}\right|}+\left(\begin{array}{c}
+ \\
+ \\
-
\end{array}\right)\left[\left(1+\frac{1}{x_{e}^{2}}\right)\left(\frac{1}{y_{e}^{2}}+\frac{1}{z_{e}^{2}}\right)\right]\right\}
$$

where

$$
z_{e} \equiv 1-x_{e}-y_{e}
$$

The normal-mode frequencies $\Omega$ and matrices $a$ of normal mode vectors that are needed in the above formulas are

$$
\begin{aligned}
\Omega_{\mathrm{i}}\left(x_{1}, x_{2}, x_{3}, x_{4}\right) & =\sqrt{\frac{-i \hat{q}}{2 E}\left(\frac{1}{x_{1}}+\frac{1}{x_{4}}\right)}, \\
\Omega_{\mathrm{f}}^{\mathrm{seq}}\left(x_{1}, x_{2}, x_{3}, x_{4}\right) & =\sqrt{\frac{-i \hat{q}}{2 E}\left(\frac{1}{x_{2}}+\frac{1}{x_{3}}\right)}, \\
\Omega_{+} & =\sqrt{-\frac{i \hat{q}}{2 E}\left(\frac{1}{x_{1}}+\frac{1}{x_{2}}+\frac{1}{x_{3}}+\frac{1}{x_{4}}\right)}, \\
a_{\mathrm{y}} & =\left[\left(-x_{1} x_{2} x_{3} x_{4}\right)\left(\frac{1}{x_{1}}+\frac{1}{x_{2}}+\frac{1}{x_{3}}+\frac{1}{x_{4}}\right) E\right]^{-1 / 2}\left(\begin{array}{cc}
\sqrt{\frac{x_{2} x_{3}}{-x_{1} x_{4}}} & 1 \\
-\sqrt{\frac{-x_{1} x_{4}}{x_{2} x_{3}}} & 1
\end{array}\right),
\end{aligned}
$$


and

$$
a_{\overline{\mathrm{x}}}^{\mathrm{seq}}=\left(\begin{array}{ll}
0 & 1 \\
1 & 0
\end{array}\right) a_{\mathrm{y}} .
$$

We have written the $\Omega$ 's and $a$ 's in general terms above, rather than plugging in the specific values of $\left(x_{1}, x_{2}, x_{3}, x_{4}\right)$ used in (A.14), because the more general form will later be useful for other types of diagrams.

\section{A.2.2 Diagrams with one instantaneous vertex}

The "(I)" piece of our result (A.11) is the contribution from diagrams (e-g) of figure 7 . Paralleling the notation of ACI4 [10] as closely as possible,

$$
\begin{aligned}
& {\left[\frac{d \Gamma}{d x_{e} d y_{e}}\right]_{(I)}=2 N_{\mathrm{f}} \mathcal{A}_{I}\left(x_{e}, y_{e}\right)} \\
& \mathcal{A}_{I}\left(x_{e}, y_{e}\right) \equiv \int_{0}^{\infty} d(\Delta t) 2 \operatorname{Re}\left(B_{I}\left(x_{e}, y_{e}, \Delta t\right)\right), \\
& B_{I}\left(x_{e}, y_{e}, \Delta t\right)=\frac{4\left(x_{e} y_{e}\left(1-x_{e}-y_{e}\right)\right)^{1 / 2}}{\left(1-x_{e}\right)^{2}}\left[D_{I}\left(\hat{x}_{1}, \hat{x}_{2}, \hat{x}_{3}, \hat{x}_{4}, \zeta, \Delta t\right)\right. \\
& \left.+D_{I}\left(-\hat{x}_{3},-\hat{x}_{4},-\hat{x}_{1},-\hat{x}_{2}, \zeta, \Delta t\right)\right] \\
& =\frac{4\left(x_{e} y_{e}\left(1-x_{e}-y_{e}\right)\right)^{1 / 2}}{\left(1-x_{e}\right)^{2}}\left[D_{I}\left(-1, y_{e}, 1-x_{e}-y_{e}, x_{e}, \zeta, \Delta t\right)\right. \\
& \left.+D_{I}\left(-\left(1-x_{e}-y_{e}\right),-x_{e}, 1,-y_{e}, \zeta, \Delta t\right)\right], \\
& D_{I}\left(x_{1}, x_{2}, x_{3}, x_{4}, \zeta, \Delta t\right)=-\frac{\alpha_{\mathrm{EM}}^{2} M_{\mathrm{f}}^{\mathrm{seq}}}{16 \pi^{2} E}\left(-x_{1} x_{2} x_{3} x_{4}\right) \zeta \frac{\Omega_{+} \csc \left(\Omega_{+} \Delta t\right)}{\Delta t} \frac{Y_{\overline{\mathrm{x}}}^{\mathrm{seq}}}{X_{\overline{\mathrm{x}}}^{\mathrm{seq}}},
\end{aligned}
$$

where

$$
\zeta=\frac{\left(1+\left|x_{e}\right|\right)\left(\left|y_{e}\right|+\left|z_{e}\right|\right)}{\left(1-x_{e}\right)^{3}\left(x_{e} y_{e} z_{e}\right)^{3 / 2}} .
$$

$M_{\mathrm{f}}^{\mathrm{seq}}, \Omega_{\mathrm{f}}^{\mathrm{seq}}$, and $\Omega_{+}$are given here by the previous general formulas (A.19), (A.25), and (A.26), for use in (A.21b) for $X_{\overline{\mathrm{x}}}^{\text {seq }}$ and $Y_{\overline{\mathrm{x}}}^{\text {seq }}$.

Later, we will need to refer separately to the contributions of the three diagrams (e-g). The above formula for $\left[d \Gamma / d x_{e} d y_{e}\right]_{(I)}$ can be decomposed as

$$
\left[\frac{d \Gamma}{d x_{e} d y_{e}}\right]_{(I)}=2 \operatorname{Re}\left[\frac{d \Gamma}{d x_{e} d y_{e}}\right]_{(\mathrm{e})}+2 \operatorname{Re}\left[\frac{d \Gamma}{d x_{e} d y_{e}}\right]_{(\mathrm{f})}+2 \operatorname{Re}\left[\frac{d \Gamma}{d x_{e} d y_{e}}\right]_{(\mathrm{g})}
$$

where

$$
\begin{aligned}
& 2 \operatorname{Re}\left[\frac{d \Gamma}{d x_{e} d y_{e}}\right]_{(\mathrm{e})}=\text { Eqs. (A.29) using only the first } D_{I} \text { term in (A.29c); } \\
& 2 \operatorname{Re}\left[\frac{d \Gamma}{d x_{e} d y_{e}}\right]_{(\mathrm{f})}=\text { Eqs. (A.29) using only the second } D_{I} \text { term in (A.29c); } \\
& 2 \operatorname{Re}\left[\frac{d \Gamma}{d x_{e} d y_{e}}\right]_{(\mathrm{g})}=0 .
\end{aligned}
$$




\section{A.2.3 Diagrams with two instantaneous vertices}

The "(II)" piece of our result (A.11) is the contribution from diagram (d) of figure 7, which gives

$$
\left[\frac{d \Gamma}{d x_{e} d y_{e}}\right]_{(I I)}=2 \operatorname{Re}\left[\frac{d \Gamma}{d x_{e} d y_{e}}\right]_{(\mathrm{d})}=\frac{4 N_{\mathrm{f}} \alpha_{\mathrm{EM}}^{2}}{\pi^{2}} \frac{x_{e} y_{e} z_{e}}{\left(1-x_{e}\right)^{4}} \operatorname{Re}\left(i \Omega_{+}\right) \ln 2 .
$$

This completes the set of formulas needed to numerically evaluate $\Delta\left[d \Gamma / d x_{e} d y_{e}\right]_{e \rightarrow e \bar{e} e}$.

\section{A.3 NLO corrections to single splitting $e \rightarrow \gamma e$}

We will decompose the contributions of figure 8 to single splitting $e \rightarrow \gamma e$ as

$$
\left[\frac{\Delta d \Gamma}{d x_{e}}\right]_{e \rightarrow \gamma e}^{\mathrm{NLO}}=2 \operatorname{Re}\left\{\left[\frac{\Delta d \Gamma}{d x_{e}}\right]_{(\mathrm{h}+\mathrm{i}+\mathrm{j})}+\left[\frac{d \Gamma}{d x_{e}}\right]_{(\mathrm{k})}+\left[\frac{d \Gamma}{d x_{e}}\right]_{(\mathrm{l})}+\left[\frac{d \Gamma}{d x_{e}}\right]_{(\mathrm{m})}+\left[\frac{d \Gamma}{d x_{e}}\right]_{(\mathrm{n})}\right\} .
$$

By the back-end transformation (4.1),

$$
\begin{aligned}
2 \operatorname{Re}\left[\frac{\Delta d \Gamma}{d x_{e}}\right]_{(\mathrm{h}+\mathrm{i}+\mathrm{j})} & =-\int_{0}^{1-x_{e}} d y_{e}\left[\frac{\Delta d \Gamma}{d x_{e} d y_{e}}\right]_{\mathrm{seq}}, \\
2 \operatorname{Re}\left[\frac{d \Gamma}{d x_{e}}\right]_{(\mathrm{l})} & =-\int_{0}^{1-x_{e}} d y_{e} 2 \operatorname{Re}\left[\frac{d \Gamma}{d x_{e} d y_{e}}\right]_{(\mathrm{e})}, \\
2 \operatorname{Re}\left[\frac{d \Gamma}{d x_{e}}\right]_{(\mathrm{n})} & =0,
\end{aligned}
$$

where the integrands on the right-hand side are specified in (A.12), (A.32) and implicitly (A.34). By the combined front- and back-end transformation (4.6),

$$
2 \operatorname{Re}\left[\frac{d \Gamma}{d x_{e}}\right]_{(\mathrm{m})}=+\int_{0}^{1-x_{e}} d y_{e}\left\{2 \operatorname{Re}\left[\frac{d \Gamma}{d x_{e} d y_{e}}\right]_{(\mathrm{e})} \text { with }\left(x_{e}, y_{e}, E\right) \rightarrow\left(\frac{-y_{e}}{z_{e}}, \frac{-x_{e}}{z_{e}}, z_{e} E\right)\right\}
$$

with $2 \operatorname{Re}\left[d \Gamma / d x_{e} d y_{e}\right]_{(\mathrm{e})}$ again given by (A.32).

Finally, diagram $(\mathrm{k})$ is computed in appendices $\mathrm{F}$ and $\mathrm{G}$, giving

$$
\begin{aligned}
2 \operatorname{Re}\left[\frac{d \Gamma}{d x_{e}}\right]_{(\mathrm{k})} & 2 \operatorname{Re}\left\{-\frac{N_{\mathrm{f}} \alpha_{\mathrm{EM}}}{3 \pi}\left[\frac{d \Gamma}{d x_{e}}\right]_{x \bar{x}}\left(\ln \left(\frac{\mu^{2}}{\left(1-x_{e}\right) E \Omega_{\mathrm{i}} \operatorname{sgn} M_{\mathrm{i}}}\right)+\gamma_{\mathrm{E}}-2 \ln 2+\frac{5}{3}\right)\right\} \\
& +\int_{0}^{1-x_{e}} d y_{e} 2 \operatorname{Re}\left[\frac{d \Gamma}{d x_{e} d y_{e}}\right]_{x y y \bar{x}}^{(\text {subtracted })}
\end{aligned}
$$

where the leading-order $x \bar{x}$ diagram is

$$
\left[\frac{d \Gamma}{d x_{e}}\right]_{x \bar{x}}=\frac{\alpha_{\mathrm{EM}}}{2 \pi} P_{e \rightarrow e}\left(x_{e}\right) i \Omega_{\mathrm{i}} \operatorname{sgn} M_{\mathrm{i}}
$$


The "subtracted" rate above is

$$
\begin{aligned}
& {\left[\frac{d \Gamma}{d x_{e} d y_{e}}\right]_{x y y \bar{x}}^{\text {(subtracted) }}=-\frac{N_{\mathrm{f}} \alpha_{\mathrm{EM}}^{2} M_{\mathrm{i}}^{2}}{16 \pi^{4} E^{2}}\left(-\hat{x}_{1} \hat{x}_{2} \hat{x}_{3} \hat{x}_{4}\right) \int_{0}^{\infty} d(\Delta t)[} \\
& \frac{\Omega_{+} \csc \left(\Omega_{+} \Delta t\right)}{\Delta t}\left\{\left(\bar{\beta} Y_{\mathrm{y}}^{2}+\bar{\gamma} \bar{Y}_{\mathrm{yy}^{\prime}} Y_{\mathrm{yy}^{\prime}}\right) I_{0}^{\text {new }}+(2 \bar{\alpha}+\bar{\beta}+\bar{\gamma}) Z_{\mathrm{yy}} I_{1}^{\text {new }}\right. \\
& +\left[(\bar{\alpha}+\bar{\gamma}) Y_{\mathrm{y}}^{2}+(\bar{\alpha}+\bar{\beta}) \bar{Y}_{\mathrm{yy}^{\prime}} Y_{\mathrm{yy}^{\prime}}\right] I_{2}^{\text {new }} \\
& \left.-(\bar{\alpha}+\bar{\beta}+\bar{\gamma})\left(\bar{Y}_{\mathrm{yy}} Y_{\mathrm{y}} I_{3}^{\text {new }}+Y_{\mathrm{y}} Y_{\mathrm{yy}} I_{4}^{\text {new }}\right)\right\} \\
& \left.-(2 \bar{\alpha}+\bar{\beta}+\bar{\gamma}) \frac{\hat{x}_{2} \hat{x}_{3}}{\hat{x}_{1} \hat{x}_{2}} \mathcal{D}_{2}^{(\mathbb{I})}\right]
\end{aligned}
$$

and

$$
\mathcal{D}_{2}^{(\mathbb{I})}(\Delta t)=2 \pi^{2}\left[\frac{\ln \left(2 i \Omega_{\mathrm{i}} \Delta t \operatorname{sgn} M_{\mathrm{i}}\right)}{(\Delta t)^{2}}-i \Omega_{\mathrm{i}}^{3} \Delta t \csc ^{2}\left(\Omega_{\mathrm{i}} \Delta t\right) \operatorname{sgn} M_{\mathrm{i}}\right] .
$$

Here the $I_{n}^{\text {new }}$ are the same as the $I_{n}^{\text {seq }}$ of (A.20) except that the $(X, Y, Z)^{\text {seq }}$ there are replaced by

$$
\begin{aligned}
\left(\begin{array}{cc}
X_{\mathrm{y}}^{\text {new }} & Y_{\mathrm{y}}^{\text {new }} \\
Y_{\mathrm{y}}^{\text {new }} & Z_{\mathrm{y}}^{\text {new }}
\end{array}\right)= & \left(\begin{array}{cc}
X_{\mathrm{y}^{\prime}}^{\text {new }} & Y_{\mathrm{y}^{\prime}}^{\text {new }} \\
Y_{\mathrm{y}^{\prime}}^{\text {new }} & Z_{\mathrm{y}^{\prime}}^{\text {new }}
\end{array}\right) \equiv\left(\begin{array}{cc}
\left|M_{\mathrm{i}}\right| \Omega_{\mathrm{i}} & 0 \\
0 & 0
\end{array}\right)-i a_{\mathrm{y}}^{-1 \top}\left(\begin{array}{cc}
\Omega_{+} \cot \left(\Omega_{+} \Delta t\right) & 0 \\
0 & (\Delta t)^{-1}
\end{array}\right) a_{\mathrm{y}}^{-1}, \\
& \left(\begin{array}{ccc}
X_{\mathrm{yy}}^{\text {new }} & Y_{\mathrm{yy}^{\prime}}^{\text {new }} \\
Y_{\mathrm{yy}^{\prime}}^{\text {new }} & Z_{\mathrm{yy}^{\prime}}^{\text {new }}
\end{array}\right) \equiv-i a_{\mathrm{y}}^{-1 \top}\left(\begin{array}{cc}
\Omega_{+} \csc \left(\Omega_{+} \Delta t\right) & 0 \\
0 & (\Delta t)^{-1}
\end{array}\right) a_{\mathrm{y}}^{-1} .
\end{aligned}
$$

[See (F.3) if further clarification desired.] The $M$ 's, $\Omega$ 's and $a$ 's are as in section A.2.1 with $\left(x_{1}, x_{2}, x_{3}, x_{4}\right)$ set to $\left(\hat{x}_{1}, \hat{x}_{2}, \hat{x}_{3}, \hat{x}_{4}\right)=\left(-1, y_{e}, 1-x_{e}-y_{e}, x_{e}\right)$. The only reason that the factors of $\operatorname{sgn} M_{\mathrm{i}}$ in (A.41)-(A.44) are necessary is to accommodate the transformation of diagram $(\mathrm{k})$ that will later be used to evaluate diagram $(\mathrm{r})$.

The specific additive constants shown in (A.41) assume that the coupling $\alpha_{\mathrm{EM}}$ used in the leading-order calculation (A.4) of single splitting is MS-bar $\alpha_{\mathrm{EM}}(\mu)$. To use a different renormalization scheme, one would need to convert (A.41) accordingly, but nothing else would change.

\section{A.4 NLO corrections to single splitting $\gamma \rightarrow e \bar{e}$}

We will decompose the contributions of figure 9 to single splitting $\gamma \rightarrow e \bar{e}$ as

$$
\left[\frac{\Delta d \Gamma}{d y_{e}}\right]_{\gamma \rightarrow e \bar{e}}^{\mathrm{NLO}}=2 \operatorname{Re}\left\{\left[\frac{\Delta d \Gamma}{d y_{e}}\right]_{(\mathrm{o}+\mathrm{p}+\mathrm{q})}+\left[\frac{d \Gamma}{d y_{e}}\right]_{(\mathrm{r})}+\left[\frac{d \Gamma}{d y_{e}}\right]_{(\mathrm{s})}+\left[\frac{d \Gamma}{d y_{e}}\right]_{(\mathrm{t})}+\left[\frac{d \Gamma}{d y_{e}}\right]_{(\mathrm{u})}\right\} .
$$

By the front-end transformation (4.3),

$$
\begin{aligned}
2 \operatorname{Re}\left[\frac{\Delta d \Gamma}{d y_{e}}\right]_{(\mathrm{o}+\mathrm{p}+\mathrm{q})} & =-N_{\mathrm{f}} \int_{0}^{1} d x_{e}\left\{\left[\frac{\Delta d \Gamma}{d x_{e} d y_{e}}\right]_{\mathrm{seq}} \text { with (A.50) below }\right\} \\
2 \operatorname{Re}\left[\frac{d \Gamma}{d y_{e}}\right]_{(\mathrm{s})} & =-N_{\mathrm{f}} \int_{0}^{1} d x_{e}\left\{2 \operatorname{Re}\left[\frac{d \Gamma}{d x_{e} d y_{e}}\right]_{(\mathrm{f})} \text { with (A.50) below }\right\} \\
2 \operatorname{Re}\left[\frac{d \Gamma}{d y_{e}}\right]_{(\mathrm{u})} & =0
\end{aligned}
$$


where

$$
\left(x_{e}, y_{e}, E\right) \rightarrow\left(\frac{-x_{e}}{1-x_{e}}, \frac{y_{e}}{1-x_{e}},\left(1-x_{e}\right) E\right) .
$$

By front- and back-end transformation (4.4),

$$
2 \operatorname{Re}\left[\frac{d \Gamma}{d y_{e}}\right]_{(\mathrm{t})}=N_{\mathrm{f}} \int_{0}^{1} d x_{e}\left\{2 \operatorname{Re}\left[\frac{d \Gamma}{d x_{e} d y_{e}}\right]_{(\mathrm{f})} \text { with }\left(x_{e}, y_{e}, E\right) \rightarrow\left(\frac{-\left(1-y_{e}\right)}{y_{e}}, \frac{x_{e}}{y_{e}}, y_{e} E\right)\right\} .
$$

Finally, the result (A.41) for diagram (k) can be transformed using (4.40) to ${ }^{30}$

$$
\begin{aligned}
& 2 \operatorname{Re}\left[\frac{d \Gamma}{d y_{e}}\right]_{(\mathrm{r})}=2 \operatorname{Re}\left\{-\frac{N_{\mathrm{f}} \alpha_{\mathrm{EM}}}{3 \pi}\left[\frac{d \Gamma}{d y_{e}}\right]_{y \bar{y}}^{\gamma \rightarrow e \bar{e}}\left(\ln \left(\frac{\mu^{2}}{E \Omega_{0}^{\gamma \rightarrow e \bar{e}}}\right)-i \pi+\gamma_{\mathrm{E}}-2 \ln 2+\frac{5}{3}\right)\right\} \\
& \quad+N_{\mathrm{f}} \int_{0}^{1} d x_{e}\left\{2 \operatorname{Re}\left[\frac{d \Gamma}{d x_{e} d y_{e}}\right]_{x y y \bar{x}}^{(\text {subtracted })} \text { with }\left(x_{e}, y_{e}, E\right) \rightarrow\left(\frac{-y_{e}}{1-y_{e}}, \frac{x_{e}}{1-y_{e}},\left(1-y_{e}\right) E\right)\right\}
\end{aligned}
$$

where

$$
\left[\frac{d \Gamma}{d y_{e}}\right]_{y \bar{y}}=\frac{N_{\mathrm{f}} \alpha_{\mathrm{EM}}}{2 \pi} P_{\gamma \rightarrow e \bar{e}}\left(y_{e}\right) i \Omega_{0}^{\gamma \rightarrow e \bar{e}}
$$

is the amplitude for which $2 \operatorname{Re}[\cdots]$ gives the leading-order pair production rate (A.8).

\section{B Similarities and dissimilarities with refs. [23, 25]}

Refs. [23, 25] study processes somewhat similar to ours but in the context of next-toleading-order DIS in the dipole approximation appropriate to studying small- $x$ physics. As is standard, one can use the optical theorem to relate DIS cross-sections to the selfenergy of the virtual photon as depicted at leading order, for example, by the time-ordered diagram in figure 32a. In their application to small- $x$ physics, the medium is very thin compared to the formation time, and we've depicted its extent by the very thin gray region in figure 32a. Figure $32 \mathrm{~b}$ shows the same process but now drawn using the conventions that we have used in figure $7 .{ }^{31}$ In this paper, however, our explicit calculations are for the case where the medium is thick compared to the relevant formation times, and so would be analogous to figure $32 \mathrm{c}$ rather than figure $32 \mathrm{~b}$.

The photon in DIS is virtual, with a virtuality $Q^{2}$ that should not be ignored. In contrast, in our calculations of in-medium showering, we approximate the initial highenergy particle in figures 7-9 as on-shell, with negligible mass or virtuality.

\footnotetext{
${ }^{30}$ See appendix $\mathrm{H}$ for technicalities on getting the first term in (A.52) from the transformation of (A.41). Also, we have not bothered to write any general $\operatorname{sgn} M$ factors in (A.52) that would allow this result to in turn be transformed back again to diagram $(\mathrm{k})$. Instead, here we have just specialized to the specific values of $\operatorname{sgn} M$ of diagram (r).

${ }^{31}$ In case the reader is wondering how the overall sign matches up between figure $32 \mathrm{a}$ and $32 \mathrm{~b}$ : in our formalism, the red portion of the diagram represents the conjugate amplitude and so evolves with $e^{+i H t}$ instead of $e^{-i H t}$. When doing time-ordered perturbation theory, the sign difference in $e^{\mp i H t}$ manifests as a sign difference between red vertices and blue vertices in our diagram.
} 


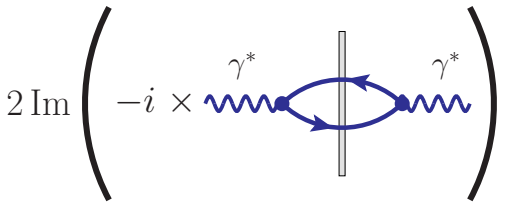

(a)

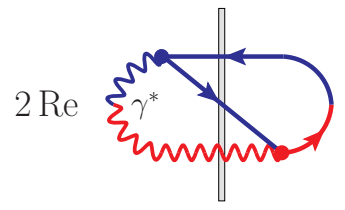

(b)

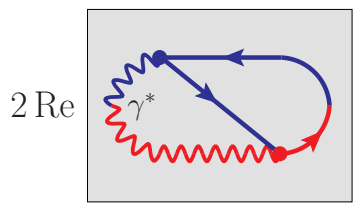

(c)

Figure 32. (a) The imaginary part of the leading-order (LO) time-ordered diagram for the virtual photon self-energy for an extremely thin medium, as in the LO piece of the NLO results in refs. [23, 25]. (b) The same, but drawn in the conventions [7] of our figure 7. (c) What the analogous process would be in the thick-medium limit considered in this paper. In all cases, the gray area denotes the longitudinal size of the medium.

Yet another difference is that refs. [23, 25] write their final answers explicitly in terms of Wilson lines running through the medium, which should be averaged over medium fluctuations. Because the medium is thin, these Wilson lines do not have time to move transversely as they cross the medium. For thick media, the paths inside the medium do move transversely, and this dynamics is incorporated in our treatment of the LPM effect [7] (based on Zakharov's picture [20, 21]) in the language of two-dimensional quantum mechanics with a non-Hermitian potential energy. This is an approximation that relies on correlation lengths in the medium being small compared to both formation times and the thickness of the medium. Finally, our explicit calculation in this paper further takes this potential to be given by the $\hat{q}$ approximation, appropriate for the LPM effect at high energy (but see appendix C).

\section{$\mathrm{C} \quad \hat{q}$ in $\mathrm{QCD}$ and QED}

In this appendix, we mention some qualitative differences between QCD and QED concerning $\hat{q}$ and its logarithmic dependence on energy at high energy. Though the development of this paper does not depend on the medium itself being weakly-coupled, we start by discussing that case.

\section{C.1 $\hat{q}$ for weakly-coupled systems}

For weak coupling, $\hat{q}$ is given by

$$
\hat{q}=\int d^{2} q_{\perp} q_{\perp}^{2} \frac{d \Gamma_{\mathrm{el}}}{d^{2} q_{\perp}}
$$

where $d \Gamma_{\mathrm{el}} / d^{2} q_{\perp}$ is the rate of elastic scattering from the medium for momentum transfer $\boldsymbol{q}_{\perp}$ perpendicular to the direction of motion of the high-energy particle. Coulomb interactions give

$$
\frac{d \Gamma_{\mathrm{el}}}{d^{2} q_{\perp}} \propto \frac{\alpha^{2}}{q_{\perp}^{4}}
$$


for $q_{\perp}$ large compared to the inverse electric screening length $\xi^{-1}$ of the medium. ${ }^{32}$ For fixed small coupling $\alpha$, this behavior leads to a logarithmic UV divergence in the integral (C.1) for $\hat{q}$ in both QED and QCD:

$$
\hat{q} \propto \int_{\sim \xi^{-1}}^{\infty} d\left(q_{\perp}^{2}\right) \frac{\alpha^{2}}{q_{\perp}^{2}}
$$

One may then define $\hat{q}(\Lambda)$ as a function of some UV cut-off $\Lambda$ on $q_{\perp}$. It turns out that the LPM calculation of the rate for splitting processes such as hard bremsstrahlung depends on $\hat{q}\left(Q_{\perp}\right)$ where $Q_{\perp}$ is the order of magnitude of the total $q_{\perp}$ transferred to the high-energy particle during a formation time. As we'll review below, this effect (though not in this language) goes all the way back to the results of Migdal [3]. ${ }^{33}$

That fact notwithstanding, it is interesting that QCD $\hat{q}(\infty)$ as calculated by (C.3) is actually convergent if one accounts for the running of the coupling as $\alpha=\alpha\left(q_{\perp}\right)$ in this particular calculation. The slow decrease of $\alpha$ with increasing momentum due to asymptotic freedom is just enough to then make the integral (C.3) convergent. ${ }^{34}$ In contrast, the running of the coupling in QED makes the divergence slightly worse since $\alpha_{\mathrm{EM}}\left(q_{\perp}\right)$ grows with momentum.

But this apparent conclusion that $\hat{q}(\infty)$ is finite for QCD, however, is an artifact of ignoring other higher-order corrections (besides running of the coupling) to the leadingorder analysis (C.3), as we now review.

\section{C.2 Small- $x$ logs in QCD}

In this paper, we have looked at splitting processes such as high-energy bremsstrahlung, which are suppressed by a factor of $\alpha_{\mathrm{S}}\left(Q_{\perp}\right)$, which is small at high enough energy even if the medium itself is strongly coupled, i.e. even if $\alpha_{\mathrm{S}}\left(\xi^{-1}\right)$ is large. Liou, Mueller, and $\mathrm{Wu}[34]$ have shown that the contribution of high-energy gluon bremsstrahlung to $\hat{q}$ in QCD is enhanced by a double logarithm, arising from emission of nearly-collinear gluons with longitudinal momenta between the medium scale and the high-energy scale $E$. This double $\log$ is related to double logs that occur in small- $x$ physics. At large energy, the double logarithm compensates for the small $\alpha_{\mathrm{s}}\left(Q_{\perp}\right)$ and so QCD $\hat{q}(\infty)$ is divergent after all, even after resummation of leading logarithms at all orders. Refs. [4-6] extended this analysis to the use of $\hat{q}$ in the context of calculations of high-energy splitting rates. Ref. [4] argues that the effective value of $\hat{q}$ in splitting calculations scales like $L^{2 \sqrt{\bar{\alpha}_{\mathrm{s}}}}$ for large energy $E$, with $\bar{\alpha}_{\mathrm{s}} \equiv N_{\mathrm{c}} \alpha_{\mathrm{s}} / \pi$, and where $L$ in the present context (infinite medium) means the formation length. Since $L$ scales like $L \sim E^{1 / 2}$ (for fixed $x$ and small $\bar{\alpha}_{\mathrm{s}}$ ), that gives $\hat{q}_{\mathrm{eff}} \sim E^{\sqrt{\bar{\alpha}_{\mathrm{s}}}}$, which diverges as $E \rightarrow \infty$.

\section{C.3 The upshot for this paper}

Whether we are talking about QED or QCD, there is some sort of logarithmic dependence of $\hat{q}\left(Q_{\perp}\right)$ on energy. The $\hat{q}$ in this paper should be fixed to the one appropriate for the

\footnotetext{
${ }^{32}$ For a precise leading-order analysis of $\hat{q}$ in the case of ultra-relativistic plasmas, including screening effects with all the finicky details, see refs. [30, 31].

${ }^{33}$ For QCD, this observation can be found in the discussion of eq. (2.19) of ref. [29].

${ }^{34} \mathrm{See}$, for example, the discussion in ref. [32] or section 2.C of ref. [33].
} 
energy $E$. By making the approximation that we can describe scattering from the medium in terms of a fixed effective value of $\hat{q}$, we are ignoring one class of sub-leading corrections. In the long term, one should be able to do a more complete calculation. In the medium term, we will sidestep this particular issue in forthcoming work [16] by looking at certain characteristics of high-energy showers that are not sensitive to the precise value of $\hat{q}$.

\section{C.4 The Coulomb logarithm in Migdal's QED result}

Since this is nominally a paper about QED (albeit large- $N_{\mathrm{f}}$ QED), it may be helpful to relate our notation and the discussion of Coulomb logs above to the early results by Migdal [3] for high-energy QED showering off of a medium made up of atoms, a useful summary of which can be found in the review by Klein [35]. Migdal's explicit solutions to his equations rely on assuming that Coulomb logarithms are large, and he does not try to precisely compute the constants under the logarithms. In the case of significant LPM suppression (what Migdal would call $s \ll 1$ ), Migdal's results for $e \rightarrow \gamma e$ can be rewritten in the following form at the same leading-log order: ${ }^{35}$

$$
\frac{d \Gamma_{\mathrm{LPM}}}{d x_{\gamma}}=n \frac{d \sigma_{\mathrm{LPM}}}{d x_{\gamma}} \simeq \frac{\alpha}{\pi} P_{e \rightarrow \gamma}\left(x_{\gamma}\right) \operatorname{Re}(i \Omega)
$$

with $P_{e \rightarrow \gamma}\left(x_{\gamma}\right)$ the DGLAP splitting function $\left[1+\left(1-x_{\gamma}\right)^{2}\right] / x_{\gamma}$,

$$
\Omega=\sqrt{\frac{-i x_{\gamma} \hat{q}_{\mathrm{eff}}\left(|\hat{q} / \Omega|^{1 / 2}\right)}{2\left(1-x_{\gamma}\right) E}},
$$

and

$$
\hat{q}_{\mathrm{eff}}\left(b^{-1}\right)=8 \pi Z^{2} \alpha^{2} n \begin{cases}\ln \left(\frac{a_{Z}}{b}\right), & R_{\mathrm{A}} \lesssim b \ll m_{e}^{-1} ; \\ \ln \left(\frac{a_{Z}}{R_{A}}\right), & b \lesssim R_{A} .\end{cases}
$$

Physically, the argument of $\hat{q}_{\text {eff }}$ in (C.5) represents $Q_{\perp} \sim \sqrt{\hat{q}} t_{\text {form }}$ (from the definition of $\hat{q}$ ), remembering that the frequency $\Omega$ is of order $1 / t_{\text {form }}$ and that rough approximations are all that are needed for the argument of the logarithm in (C.6) for a leading-log analysis. The $\hat{q}$ in the argument of $\hat{q}_{\text {eff }}$ can be interpreted as self-consistently $\hat{q}_{\text {eff }}$ itself, but it does not matter at leading-log order. In (C.6), our notation $\hat{q}_{\mathrm{eff}}\left(b^{-1}\right)$ is motivated by $b \sim 1 / Q_{\perp}$ because we find it more convenient to express the right-hand side of (C.6) in terms of transverse distance scales. Above, $m_{e}$ is the electron mass, $R_{A}$ is the nuclear radius (which Migdal somewhat obscures by approximating $\left.R_{A} \simeq 0.5 Z^{1 / 3} \alpha / m_{e}\right), a_{Z}$ is the length scale for screening of the nucleus's Coulomb field by atomic electrons (for which Migdal uses the Thomas-Fermi approximation $a_{Z} \simeq Z^{-1 / 3} a_{0}$ ), and $Z$ is atomic number. The case $b \gg m_{e}^{-1}$ not shown above would correspond to no LPM suppression (what Migdal would call $s \gg 1$ ),

\footnotetext{
${ }^{35}$ It is easiest to take Migdal's results from Klein (72-77) [35] because, among other things, Migdal is inconsistent about whether he works in units where $m_{e}=1$. In our notation, Klein's $E_{\mathrm{LPM}} / \xi(s)$ is $m_{e}^{4} / 2 \hat{q}_{\mathrm{eff}}(b)$, and Migdal and Klein's $s$ is $m_{e}^{2}|\Omega| / 8^{1 / 2} \hat{q}_{\mathrm{eff}}(b) \sim m_{e}^{2} / Q_{\perp}^{2}$. Our $x_{\gamma}$ is Klein's $k / E$, which he also calls $y$. Migdal's crisp-seeming result that $\xi(s)=2$ for $s<s_{1}$ (what we refer to as $b \lesssim R_{A}$ ) is actually an approximation based on the coincidence that $\ln \left(a_{Z} / R_{A}\right) \approx 2 \ln \left(a_{Z} / m_{e}^{-1}\right)$.
} 
and the case $b \simeq m_{e}^{-1}$ would correspond to the transition where the LPM effect is first turning on (which Migdal would call $s \sim 1$ ).

The point of writing these formulas in the above form is that (C.4) is precisely the leading-order result (A.4) in our notation. And the leading-log calculation (C.1) of $\hat{q}$ in Migdal's application is

$$
\hat{q}=\int d^{2} q_{\perp} q_{\perp}^{2} \frac{d \Gamma_{\mathrm{el}}}{d^{2} q_{\perp}}=n \int \frac{d^{2} q_{\perp}}{(2 \pi)^{2}} \frac{Z^{2} g^{4}}{q_{\perp}^{4}}=8 \pi Z^{2} \alpha^{2} n \int_{\sim a_{Z}^{-1}}^{\Lambda} \frac{d q_{\perp}}{q_{\perp}}=8 \pi Z^{2} \alpha^{2} n \ln \left(\frac{a_{Z}}{\Lambda^{-1}}\right),
$$

where $n$ is the density of atoms. There are now two cases to consider. (i) We mentioned previously that the scale $Q_{\perp}$ acts as a UV cut-off on the relevant value of $\hat{q}_{\text {eff }}$ for splitting calculations. Taking $\Lambda \sim Q_{\perp}$, and then rewriting $Q_{\perp}$ as $1 / b$ for the sake of expressing scales in terms of transverse distance scales instead of transverse momentum, gives the logarithm shown for the $R_{A} \lesssim b \ll m_{e}^{-1}$ case of (C.6). (ii) Ignoring the sub-structure of the nucleus (whose effects are suppressed by powers of $Z^{-1}$ except at very much higher energies $^{36}$ ), there is a UV-cutoff on the nucleus's Coulomb field at $b \sim R_{A}$. The effective cut-off for splitting calculations is therefore $\Lambda^{-1} \sim \min \left(Q_{\perp}, R_{A}^{-1}\right)$ instead of just $\Lambda \sim Q_{\perp}$, which accounts for the other case of (C.6).

\section{Diagrammatic vertex rules}

In the main text, figures 17-19 gave examples of LCPT vertex rules and the corresponding rules in our formalism. The rest of the rules that we need for the large- $N_{\mathrm{f}}$ QED diagrams of figures $7-9$ are shown explicitly in figures $33-36$. In some transformations, we must negate momentum variables $\left(x_{n}, \boldsymbol{p}_{n}\right) \rightarrow\left(-x_{n},-\boldsymbol{p}_{n}\right)$, as indicated, to account for our convention that momentum variables are negated for particles in conjugate-amplitudes (red lines in the diagrams). Transverse position variables $\boldsymbol{b}_{n}$ are unaffected. When applied to the definitions (3.1) and (3.4) of $\boldsymbol{P}_{i j}$ and $\boldsymbol{B}_{i j}$, this transformation takes $\left(\boldsymbol{P}_{i j}, \boldsymbol{B}_{i j}\right) \rightarrow\left(\boldsymbol{P}_{i j},-\boldsymbol{B}_{i j}\right)$.

The annoying part of working with the rules laid out for matrix elements is that one must be careful about Fermi statistics in the representation of states, e.g. $|e \bar{e}\rangle=-|\bar{e} e\rangle$. The boxed LCPT rule for $\bar{e} e \rightarrow \gamma$ in figure 34, for example, could just as well have been written as $\langle\mathrm{f}|\delta H| 3,2\rangle_{\text {rel }}=+g \bar{v}_{2} \ddagger_{\mathrm{f}} u_{3}$. The sign would be compensated, in a photon self-energy diagram for example, by whether the propagator between the two vertices was $\left\langle 2,3 ; t^{\prime} \mid 2,3 ; t\right\rangle$ or its negative $\left\langle 2,3 ; t^{\prime} \mid 3,2 ; t\right\rangle$. (The minus sign in the last case would be equivalent to the usual accounting where one says that fermion loops come with minus signs.)

We should also clarify a point about our formulas for $e \rightarrow e \bar{e} e$ matrix elements, such as $\langle 2,3,4|\delta H| \mathrm{i}\rangle_{\text {rel }}$ and related formulas in figures 18 and 35. In these cases, we are only

\footnotetext{
${ }^{36}$ For $R_{\mathrm{p}} \lesssim b \ll R_{A}$, where $R_{\mathrm{p}}$ is the proton radius, the $q_{\perp}$ integrand in (C.7) for $\Lambda=1 / b$ is suppressed for $q_{\perp} \ll R_{A}$ because the charges inside the nucleus do not then contribute coherently. (This suppression replaces $Z^{2}$ by $Z$ in the integrand by the time $q_{\perp}$ gets as small as $q_{\perp} \sim R_{\mathrm{p}}$.) As a result, Migdal's leading$\log$ result for what we'd call $\hat{q}_{\mathrm{eff}}(1 / b)$ does not change as $b$ drops below $R_{A}$. If one goes to energies high enough (and so $b$ small enough) to probe the substructure of the nucleons (which Migdal did not know about), then, as a matter of principle, eventually the contribution of scattering from individual quarks would become important at sufficiently small $b \ll R_{\mathrm{p}}$.
} 


$$
\begin{aligned}
& { }_{\mathrm{i}} \mathbf{L}{ }_{3}^{2}\langle 2,3|\delta H| \mathrm{i}\rangle_{\text {rel }}=-g \bar{u}_{3} \ddagger_{\mathrm{i}} v_{2}
\end{aligned}
$$

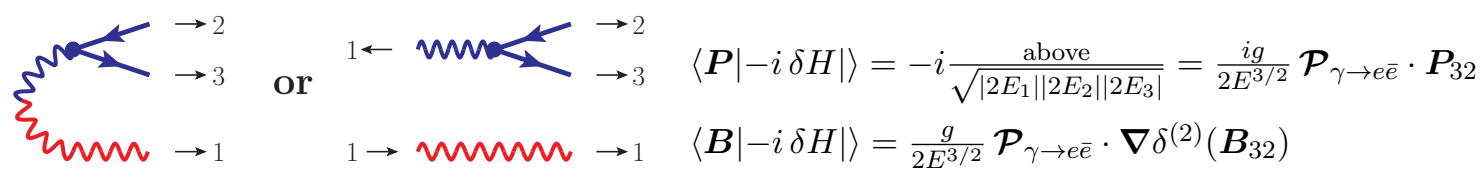

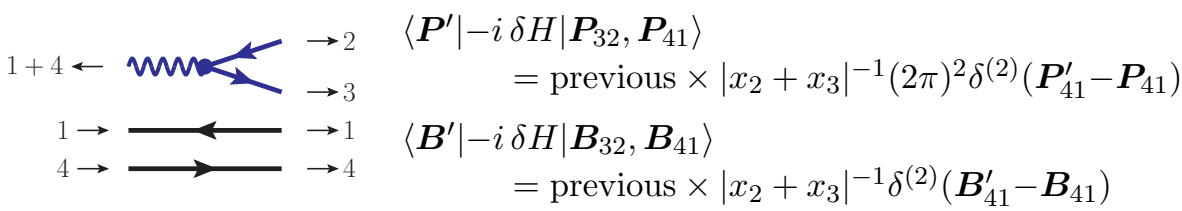

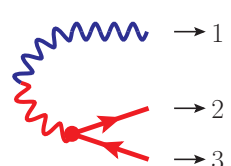

$$
\begin{aligned}
& \text { or } \begin{array}{ll}
1 \leftarrow \text { man } \\
\rightarrow 2
\end{array} \\
& \langle\boldsymbol{P}|+i \overline{\delta H}|\rangle=\langle\boldsymbol{P}|-i \delta H|\rangle_{(x, \boldsymbol{p}) \rightarrow(-x,-\boldsymbol{p})}^{*} \\
& =-\frac{i g}{2 E^{3 / 2}} \mathcal{P}_{\gamma \rightarrow e \bar{e}}^{*} \cdot \stackrel{P}{32} \\
& \langle\boldsymbol{B}|+i \overline{\delta H}|\rangle=\langle\boldsymbol{B}|-i \delta H|\rangle_{(x, \boldsymbol{b}) \rightarrow(-x, \boldsymbol{b})}^{*} \\
& =-\frac{g}{2 E^{3 / 2}} \mathcal{P}_{\gamma \rightarrow e \bar{e}}^{*} \cdot \nabla \delta^{(2)}\left(\boldsymbol{B}_{32}\right) \\
& \begin{aligned}
4 \rightarrow \longrightarrow & \longrightarrow \\
1 \rightarrow 4 \leftarrow & \rightarrow 4 \\
& \rightarrow 1 \\
& \rightarrow 2 \\
& \rightarrow 3
\end{aligned} \\
& \left\langle\boldsymbol{P}^{\prime}|+i \overline{\delta H}| \boldsymbol{P}_{32}, \boldsymbol{P}_{41}\right\rangle \\
& =\text { previous } \times\left|x_{2}+x_{3}\right|^{-1}(2 \pi)^{2} \delta^{(2)}\left(\boldsymbol{P}_{41}^{\prime}-\boldsymbol{P}_{41}\right) \\
& \left\langle\boldsymbol{B}^{\prime}|+i \overline{\delta H}| \boldsymbol{B}_{32}, \boldsymbol{B}_{41}\right\rangle \\
& =\text { previous } \times\left|x_{2}+x_{3}\right|^{-1} \delta^{(2)}\left(\boldsymbol{B}_{41}^{\prime}-\boldsymbol{B}_{41}\right) \\
& \langle|+i \overline{\delta H}| \boldsymbol{P}\rangle=\langle\boldsymbol{P}|-i \delta H|\rangle^{*}=-\frac{i g}{2 E^{3 / 2}} \boldsymbol{P}_{\gamma \rightarrow e \bar{e}}^{*} \cdot \boldsymbol{P}_{32} \\
& \langle|+i \overline{\delta H}| \boldsymbol{B}\rangle=\langle\boldsymbol{B}|-i \delta H|\rangle^{*} \\
& =\frac{g}{2 E^{3 / 2}} \mathcal{P}_{\gamma \rightarrow e \bar{e}}^{*} \cdot \nabla \delta^{(2)}\left(\boldsymbol{B}_{32}\right) \\
& \langle|-i \delta H| \boldsymbol{P}\rangle=\langle|+i \overline{\delta H}| \boldsymbol{P}\rangle_{(x, \boldsymbol{p}) \rightarrow(-x,-\boldsymbol{p})}^{*} \\
& =\frac{i g}{2 E^{3 / 2}} \boldsymbol{P}_{\gamma \rightarrow e \bar{e}} \cdot \boldsymbol{P}_{32} \\
& \langle|-i \delta H| \boldsymbol{B}\rangle=\langle|+i \overline{\delta H}| \boldsymbol{B}\rangle_{(x, \boldsymbol{b}) \rightarrow(-x, \boldsymbol{b})}^{*} \\
& =-\frac{g}{2 E^{3 / 2}} \mathcal{P}_{\gamma \rightarrow e \bar{e}}^{*} \cdot \nabla \delta^{(2)}\left(\boldsymbol{B}_{32}\right)
\end{aligned}
$$

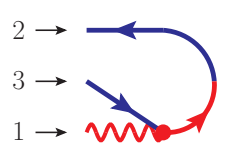

Figure 33. Like figures 17 and 19 but for pair production. We only show elements that are directly used in the diagrams of figures $7-9$.

giving the specific contribution associated with the accompanying vertex diagram. For example, the contribution from swapping the two final-state electrons (which in any case is sub-leading in $1 / N_{\mathrm{f}}$ ) is not included, and would correspond to drawing a different vertex diagram where the " 2 " and " 4 " lines were switched.

Keeping track of all the signs and different cases for matrix elements is painstaking. However, there is an equivalent way to formulate our rules for diagrams that makes it easier and a little bit more like the conventions for Feynman rules. All the cases of $1 \leftrightarrow 2$ splittings can be subsumed by the rule shown in figure 37, supplemented by a minus sign for each 

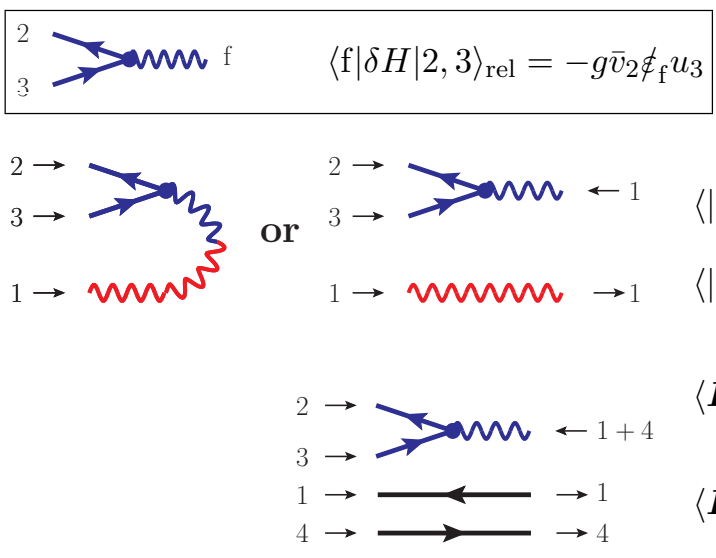

$$
\begin{aligned}
& \langle|-i \delta H| \boldsymbol{P}\rangle=-i \frac{\text { above }}{\sqrt{\left|2 E_{1}\right|\left|2 E_{2}\right|\left|2 E_{3}\right|}}=\frac{i g}{2 E^{3 / 2}} \boldsymbol{P}_{\gamma \rightarrow e \bar{e}}^{*} \cdot \boldsymbol{P}_{32} \\
& \langle|-i \delta H| \boldsymbol{B}\rangle=-\frac{g}{2 E^{3 / 2}} \mathcal{P}_{\gamma \rightarrow e \bar{e}}^{*} \cdot \nabla \delta^{(2)}\left(\boldsymbol{B}_{32}\right) \\
& \left\langle\boldsymbol{P}_{32}, \boldsymbol{P}_{41}|-i \delta H| \boldsymbol{P}^{\prime}\right\rangle \\
& \quad=\text { previous } \times\left|x_{2}+x_{3}\right|^{-1}(2 \pi)^{2} \delta^{(2)}\left(\boldsymbol{P}_{41}^{\prime}-\boldsymbol{P}_{41}\right) \\
& \left\langle\boldsymbol{B}_{32}, \boldsymbol{B}_{41}|-i \delta H| \boldsymbol{B}^{\prime}\right\rangle \\
& \quad=\text { previous } \times\left|x_{2}+x_{3}\right|^{-1} \delta^{(2)}\left(\boldsymbol{B}_{41}^{\prime}-\boldsymbol{B}_{41}\right)
\end{aligned}
$$
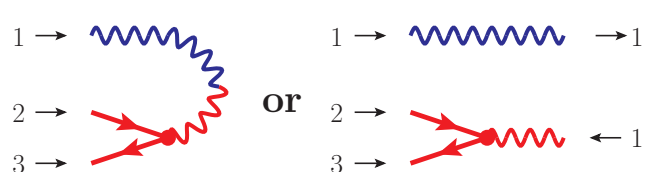

$$
\begin{gathered}
\langle|+i \overline{\delta H}| \boldsymbol{P}\rangle=\langle|-i \delta H| \boldsymbol{P}\rangle_{(x, \boldsymbol{p}) \rightarrow(-x,-\boldsymbol{p})}^{*} \\
=-\frac{i g}{2 E^{3 / 2}} \boldsymbol{P}_{\gamma \rightarrow e \bar{e}} \cdot \stackrel{\boldsymbol{P}}{32}_{32} \\
\langle|+i \overline{\delta H}| \boldsymbol{B}\rangle=\langle|-i \delta H| \boldsymbol{B}\rangle_{(x, \boldsymbol{b}) \rightarrow(-x, \boldsymbol{b})}^{*} \\
=\frac{g}{2 E^{3 / 2}} \boldsymbol{P}_{\gamma \rightarrow e \bar{e}} \cdot \boldsymbol{\nabla} \delta^{(2)}\left(\boldsymbol{B}_{32}\right)
\end{gathered}
$$

Figure 34. Like figure 33 but for inverse pair production.

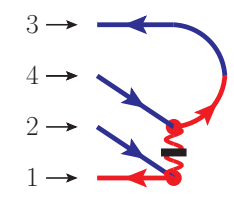

$$
\begin{aligned}
& \left\langle|+i \overline{\delta H}| \boldsymbol{P}_{34}, \boldsymbol{P}_{12}\right\rangle=\left\langle\boldsymbol{P}_{34}, \boldsymbol{P}_{12}|-i \delta H|\right\rangle^{*}=\frac{i g^{2}}{\left|x_{3}+x_{4}\right|^{3} E^{2}} \\
& \left\langle|+i \overline{\delta H}| \boldsymbol{B}_{34}, \boldsymbol{B}_{12}\right\rangle=\frac{i g^{2}}{\left|x_{3}+x_{4}\right|^{3} E^{2}} \delta^{(2)}\left(\boldsymbol{B}_{34}\right) \delta^{(2)}\left(\boldsymbol{B}_{12}\right)
\end{aligned}
$$

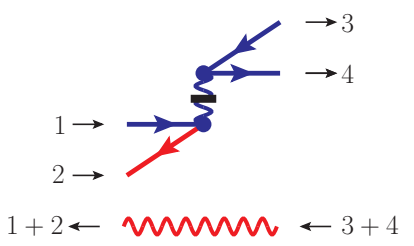

$$
\begin{aligned}
\left\langle\boldsymbol{P}^{\prime}|-i \delta H| \boldsymbol{P}\right\rangle & =-i \frac{\text { boxed }}{\sqrt{\left|2 E_{1}\right|\left|2 E_{2}\right|\left|2 E_{3}\right|\left|2 E_{4}\right|}}\left(x_{3}+x_{4}\right)^{-2}=\frac{-i g^{2}}{\left(x_{3}+x_{4}\right)^{4} E^{2}} \\
\left\langle\boldsymbol{B}^{\prime}|-i \delta H| \boldsymbol{B}\right\rangle & =\frac{-i g^{2}}{\left|x_{3}+x_{4}\right|^{3} E^{2}} \delta^{(2)}\left(\boldsymbol{B}_{34}^{\prime}\right) \delta^{(2)}\left(\boldsymbol{B}_{12}\right)
\end{aligned}
$$

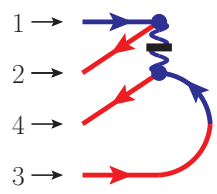

$$
\begin{aligned}
& \left\langle|-i \delta H| \boldsymbol{P}_{34}, \boldsymbol{P}_{12}\right\rangle=\left\langle\boldsymbol{P}_{34}, \boldsymbol{P}_{12}|+i \overline{\delta H}|\right\rangle_{(x, \boldsymbol{p}) \rightarrow(-x,-\boldsymbol{p})}^{*}=\frac{-i g^{2}}{\left|x_{3}+x_{4}\right|^{3} E^{2}} \\
& \left\langle|-i \delta H| \boldsymbol{B}_{34}, \boldsymbol{B}_{12}\right\rangle=\frac{-i g^{2}}{\left|x_{3}+x_{4}\right|^{3} E^{2}} \delta^{(2)}\left(\boldsymbol{B}_{34}\right) \delta^{(2)}\left(\boldsymbol{B}_{12}\right)
\end{aligned}
$$

Figure 35. The other variations of figure 18 that appear in figures 7-9. Above, "boxed" refers to the boxed LCPT rule in figure 18.

fermion loop in the interference diagrams of figures 7-9 and 31. The rule of figure 37 sometimes differs by a sign from the particular conventions of the $-i \delta H$ and $+i \overline{\delta H}$ matrix elements we have written down for individual vertices, but the supplemental minus signs for each fermion loop brings the two different procedures into agreement and also would produce the correct Fermi-statistics sign for exchange diagrams such as figure 38 (which 

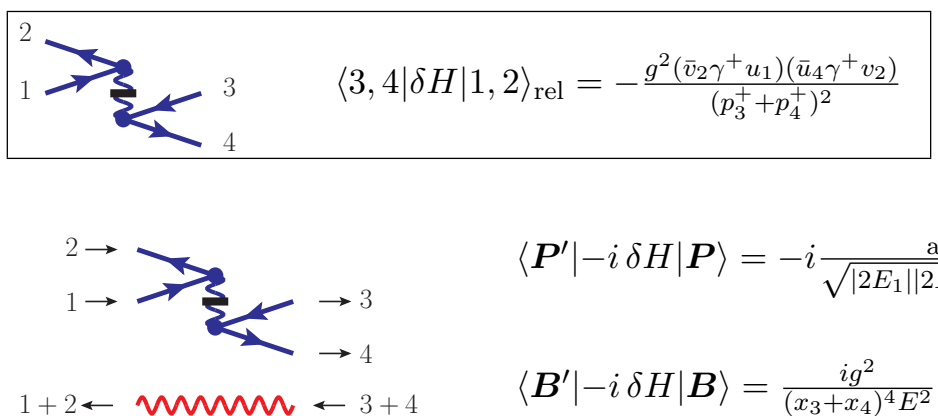

$$
\begin{aligned}
& \left\langle\boldsymbol{P}^{\prime}|-i \delta H| \boldsymbol{P}\right\rangle=-i \frac{\text { above }}{\sqrt{\left|2 E_{1}\right|\left|2 E_{2}\right|\left|2 E_{3}\right|\left|2 E_{4}\right|}}\left(x_{3}+x_{4}\right)^{-2}=\frac{i g^{2}}{\left(x_{3}+x_{4}\right)^{4} E^{2}} \\
& \left\langle\boldsymbol{B}^{\prime}|-i \delta H| \boldsymbol{B}\right\rangle=\frac{i g^{2}}{\left(x_{3}+x_{4}\right)^{4} E^{2}} \delta^{(2)}\left(\boldsymbol{B}_{34}^{\prime}\right) \delta^{(2)}\left(\boldsymbol{B}_{12}\right)
\end{aligned}
$$

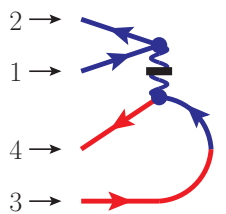

$$
\begin{aligned}
& \left\langle|-i \delta H| \boldsymbol{P}_{34}, \boldsymbol{P}_{12}\right\rangle=\frac{-i g^{2}}{\left|x_{3}+x_{4}\right|^{3} E^{2}} \\
& \left\langle|-i \delta H| \boldsymbol{B}_{34}, \boldsymbol{B}_{12}\right\rangle=\frac{-i g^{2}}{\left|x_{3}+x_{4}\right|^{3} E^{2}} \delta^{(2)}\left(\boldsymbol{B}_{23}\right) \delta^{(2)}\left(\boldsymbol{B}_{41}\right)
\end{aligned}
$$

Figure 36. Like figures 18 and 35 but for the LCPT $e \bar{e} \rightarrow e \bar{e}$ vertex.

are sub-leading in $1 / N_{\mathrm{f}}$ ). There are different ways one could assign signs to the vertices that would give the same net overall sign for interference diagrams such as figures 8-9; we've picked one of them. The rule of figure 37 has the same form as the similar rule given in AI1 [7] for the 3-gluon vertex except for details about signs. In the rule, the factor $\mathcal{P}_{\bar{e} e \gamma}\left(x_{i}, x_{j}, x_{k}\right)$ depends implicitly on the helicities $h$ of the lines as measured in the directions of the small arrows. The definition of $\mathcal{P}_{\bar{e} e \gamma}$ is as in AI1 section IV.E, adapted here for the QED case as

$$
\mathcal{P}_{i j k}^{\bar{e} e \gamma}=\frac{\boldsymbol{e}_{\left(h_{i}+h_{j}+h_{k}\right)}}{\left|x_{i} x_{j} x_{k}\right|} \sqrt{P_{h_{i}, h_{j}, h_{k}}^{\bar{e} e \gamma}\left(x_{i}, x_{j}, x_{k}\right)}
$$

with spin-dependent DGLAP splitting functions

$$
\begin{array}{r}
P_{-++}^{\bar{e} e \gamma}\left(x_{i}, x_{j}, x_{k}\right) \equiv P_{+\rightarrow++}^{e \rightarrow e \gamma}\left(-x_{i} \rightarrow x_{j} x_{k}\right)=P_{-\rightarrow-+}^{\gamma \rightarrow e \bar{e}}\left(-x_{k} \rightarrow x_{i} x_{j}\right)=\frac{x_{i}^{2}}{\left|x_{k}\right|} \\
P_{-+-}^{\bar{e} e \gamma}\left(x_{i}, x_{j}, x_{k}\right) \equiv P_{+\rightarrow+-}^{e \rightarrow e \gamma}\left(-x_{i} \rightarrow x_{j} x_{k}\right)=P_{+\rightarrow-+}^{\gamma \rightarrow e \bar{e}}\left(-x_{k} \rightarrow x_{i} x_{j}\right)=\frac{x_{j}^{2}}{\left|x_{k}\right|} \\
P_{--+}^{\bar{e} e \gamma} \equiv P_{+\rightarrow-+}^{e \rightarrow e \gamma}=P_{-\rightarrow--}^{\gamma \rightarrow e \bar{e}}=0, \\
P_{---}^{\bar{e} e \gamma} \equiv P_{+\rightarrow--}^{e \rightarrow e \gamma}=P_{+\rightarrow--}^{\gamma \rightarrow e \bar{e}}=0,
\end{array}
$$

and $\boldsymbol{e}_{ \pm} \equiv \boldsymbol{e}_{x} \pm \boldsymbol{e}_{y}$. The zeros above are a consequence of chirality conservation. As in AI1 [7], the $P\left(x_{\mathrm{i}} \rightarrow x_{j} x_{k}\right)$ are defined in terms of the usual DGLAP splitting functions by

$$
P\left(x_{\mathrm{i}} \rightarrow x_{j} x_{k}\right) \equiv\left|x_{\mathrm{i}}\right| P\left(z_{j}, z_{k}\right)
$$

where $z \equiv x / x_{\mathrm{i}}$ are the momentum fractions of the daughters relative to their immediate parent. The advantage of the $P\left(x_{i}, x_{j}, x_{k}\right)$ is that they are normalized so that they are symmetric with respect to permuting the parent with the daughters and so are the same 


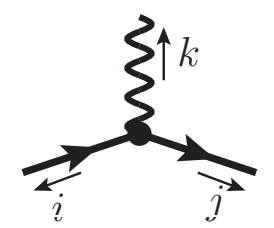

$$
\begin{aligned}
& =-\frac{g}{2 E^{3 / 2}} \mathcal{P}_{\bar{e} e \gamma}\left(x_{i}, x_{j}, x_{k}\right) \cdot \nabla \delta^{(2)}\left(\mathcal{B}_{j i}\right) \\
& \quad \times \begin{cases}+1, & \text { vertex in amplitude } \\
-1, & \text { vertex in conjugated amplitude }\end{cases}
\end{aligned}
$$

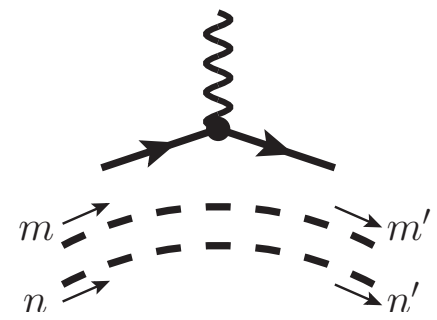

$=($ above $) \times\left|x_{m}+x_{n}\right|^{-1} \delta^{(2)}\left(\mathcal{B}_{m n}-\mathcal{B}_{m n}^{\prime}\right)$

Figure 37. (top) A general diagrammatic 3-point rule that covers all the various cases for $0 \leftrightarrow 3$ and $2 \leftrightarrow 3$ particle transitions in the interference diagrams of figures $7-9$ and 31 , provided one also includes a minus sign for every fermion loop in the interference diagram. There is no arrow of time in the drawing of the figure: each of the three lines could be initial or final ones in the corresponding matrix element. Above, $\mathcal{B}_{i j} \equiv\left(\boldsymbol{b}_{i}-\boldsymbol{b}_{j}\right) /\left(x_{i}+x_{j}\right)$, the signs of the momentum fractions $x$ are to be taken according to the flow of longitudinal momentum in the direction of the small arrows in the figure, and $\mathcal{B}_{i j}=\mathcal{B}_{j k}=\mathcal{B}_{k i}$. (bottom) The corresponding rule for $3 \leftrightarrow 4$ particle transitions, where the dashed lines represent spectators that could be fermions or photons.

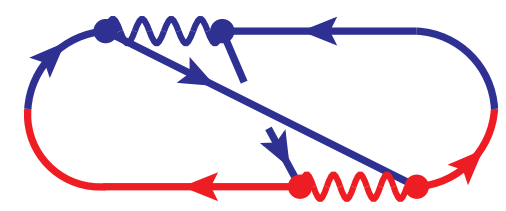

Figure 38. Example of a final-state electron exchange contribution (which is sub-leading in $1 / N_{\mathrm{f}}$ ).

for $e \rightarrow \gamma e$ and $\gamma \rightarrow e \bar{e}$, as indicated in (D.2). Eqs. (D.2) only show half of the helicity cases; the other half are given by $P_{h_{1}, h_{2}, h_{3}}=P_{-h_{1},-h_{2},-h_{3}}$ and so

$$
\mathcal{P}_{h_{1}, h_{2}, h_{3}}=\mathcal{P}_{-h_{1},-h_{2},-h_{3}}^{*}
$$

Similar rules for the instantaneous photon interactions are given by figure 39, which is similar in implementation to the 4-gluon vertex rule of ACI4 figure 10 [10].

\section{E Calculation of real double splitting (figure 7)}

The calculations of real double splitting diagrams $e \rightarrow e \bar{e} e$ proceed the same way as the $g \rightarrow g g g$ calculations of refs. [7, 8, 10] (AI1,ACI2,ACI4), with schematically very similar results.

In large $N_{\mathrm{f}}$, we distinguish the final-state electron that carries the flavor of the initialstate electron and refer to it as $x_{e}$. The electron in the pair produced from the photon (which has a different flavor in large $N_{\mathrm{f}}$ ) is referred to as $y_{e}$. Because we distinguish these 


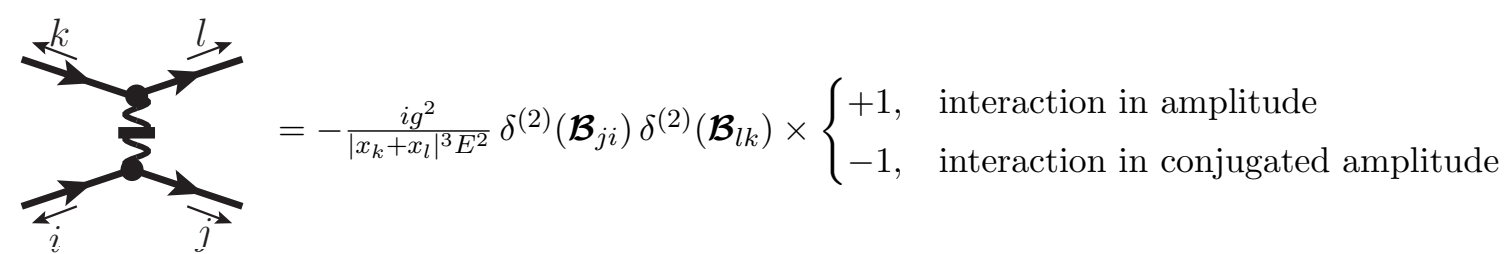

Figure 39. Similar to figure 37 but for the 4 -fermion vertex associated with longitudinal photon exchange. The rule here is normalized to cover all the cases in this paper for $0 \leftrightarrow 4$ or $2 \leftrightarrow 4$ particle transitions, which involve instantaneous $e \rightarrow e \bar{e} e$ in either the amplitude or conjugate amplitude. [We have not bothered presenting a generalized rule for $3 \rightarrow 3$ transitions such as in figures $7(\mathrm{~g})$ and $8(\mathrm{n})$ for $e \rightarrow e \bar{e} e$ and $9(\mathrm{u})$ for $e \bar{e} \rightarrow e \bar{e}$, since these diagrams give zero by symmetry arguments similar to those of ACI4 section III.B [10].]

particles in our large- $N_{\mathrm{f}}$ formulas, $\Delta d \Gamma / d x_{e} d y_{e}$ is normalized so that, in applications, final-state integrations should be performed as

$$
\int_{0}^{1} d x_{e} d y_{e} \theta\left(1-x_{e}-y_{e}\right)
$$

without any factor of $\frac{1}{2}$ for identical final-state particles.

\section{E.1 Sequential diagrams}

\section{E.1.1 Generic formulas}

The calculation of the diagrams of figure $7(\mathrm{a}-\mathrm{c})$ mimics that of the corresponding diagrams in ACI2 [8]. Using the same notation, the results can be directly taken over, except for some normalization factors and low-level details that we will get to in a moment. Adapting the results summarized in ACI4 appendix D.3 [10], ${ }^{37}$ the sum of figures $7(\mathrm{a}-\mathrm{c})$ yields the equations (A.12)-(A.19) given in our summary of results. There are a few small differences between those equations and $g \rightarrow g g g$ formulas.

- The naive translation of $C_{\mathrm{A}}^{2} \alpha_{\mathrm{s}}$ to $\mathrm{QED}$ is

$$
C_{\mathrm{A}}^{2} \alpha_{\mathrm{s}} \rightarrow N_{\mathrm{f}} \alpha_{\mathrm{EM}} \quad \text { (sequential diagrams) }
$$

since (i) the factor of $C_{\mathrm{A}}^{2}$ in QCD sequential diagrams came from $d_{\mathrm{A}}^{-1} \operatorname{tr}\left(T^{a} T^{a} T^{b} T^{b}\right)$, which is 1 in QED, and (ii) there are $N_{\mathrm{f}}$ possible flavors of the $e \bar{e}$ pair produced by the virtual photon in $e \rightarrow \gamma^{*} e \rightarrow e \bar{e} e$.

- In the QED analysis, we do not have to worry about large- $N_{\mathrm{c}}$ "color routings," which affected the choice of how to write the $g \rightarrow g g g$ version of (A.12), as described in ACI2 section 2.B.1 [8]. Each QCD diagram was the sum of two color routings, with each routing represented by $\mathcal{A}$. If we adopt the translation (E.2), that means that $\mathcal{A}$ will represent half of each QED diagram, which is the reason for the overall factor of

\footnotetext{
${ }^{37}$ The summary in ACI4 appendix D [10] incorporates some corrections [9] to the analysis of "pole" terms in the earlier paper ACI2 [8]. So it is better to take formulas from ACI4 appendix D than directly from the original ACI2 analysis.
} 
2 in (A.12). We could have instead absorbed this factor of 2 into the normalizations of $D_{\text {seq }}, F_{\text {seq }}$ and $\mathcal{A}_{\text {seq }}^{\text {pole }}$, but it seemed more convenient to normalize the lower-level formulas in exactly the same way as the QCD case.

- For $g \rightarrow g g g$, there are three identical particles in the final state, and refs. [7, 8, 10] correspondingly add together all permutations of $\mathcal{A}(x, y)$ corresponding to permuting $x, y$, and $z \equiv 1-x-y$. For $e \rightarrow e \bar{e} e$, there are two identical particles in the final state in ordinary $N_{\mathrm{f}}=1 \mathrm{QED}$, and so one might reasonably think that, analogously, the $\mathcal{A}_{\text {seq }}\left(x_{e}, y_{e}\right)$ on the right-hand side of (A.12) above should be $\mathcal{A}_{\text {seq }}\left(x_{e}, y_{e}\right)+\mathcal{A}_{\text {seq }}\left(y_{e}, x_{e}\right)$. If so, one would integrate over final-state particles as $\frac{1}{2} \int_{0}^{1} d x_{e} d y_{e} \theta\left(1-x_{e}-y_{e}\right)$ in applications of $d \Gamma / d x_{e} d y_{e}$, where the factor of $\frac{1}{2}$ would avoid double counting of identical final states. In large $N_{\mathrm{f}}$, however, we distinguish the two electrons as explained previously. To allow applications the option of tracking the fate of the initial-flavored electron, we have chosen not to include the $x_{e} \leftrightarrow y_{e}$ permutation in (A.12). When using our large- $N_{\mathrm{f}} d \Gamma / d x_{e} d y_{e}$ in applications, one should correspondingly integrate as in (E.1), without any final-state factor of $\frac{1}{2}$.

The quantities $I_{n}^{\text {seq }}$ in (A.16) are defined the same way (A.20) as in ACI4 appendix D.2 [10]. The formulas for $(X, Y, Z)^{\text {seq }}$ there and in (A.16), which are expressed in terms of the eigenfrequencies and eigenmodes of the problem, are also given in ACI4 appendix D.2 [10]. We will see below that one of the eigenfrequencies, $\Omega_{-}$, vanishes in our QED application, and so the formulas for $(X, Y, Z)^{\mathrm{seq}}$ specialize to (A.21), where

$$
a_{\mathrm{y}}=\left(\begin{array}{ll}
C_{41}^{+} & C_{41}^{-} \\
C_{23}^{+} & C_{23}^{-}
\end{array}\right)
$$

is a matrix of appropriately normalized modes of the double harmonic oscillator problem in the basis $\left(\boldsymbol{C}_{41}, \boldsymbol{C}_{23}\right)$ used by ACI2 [8] at the $y$ vertex of the $x y \bar{x} \bar{y}$ diagram, and

$$
a_{\overline{\mathrm{x}}}^{\mathrm{seq}}=\left(\begin{array}{ll}
C_{23}^{+} & C_{41}^{-} \\
C_{23}^{+} & C_{41}^{-}
\end{array}\right)=\left(\begin{array}{ll}
0 & 1 \\
1 & 0
\end{array}\right) a_{\mathrm{y}}
$$

is a permutation appropriate to the $\bar{x}$ vertex.

\section{E.1.2 QED formulas for $(\bar{\alpha}, \bar{\beta}, \bar{\gamma})$}

As in ACI2 [8], the $(\bar{\alpha}, \bar{\beta}, \bar{\gamma})$ in (A.14) are functions of $x_{e}$ and $y_{e}$ that represent various combinations of helicity-dependent DGLAP splitting functions from the vertices of figure $7 \mathrm{a}$. The relevant QED splitting functions are different from those of the $g \rightarrow g g g$ process in QCD. Performing the same calculation as in ACI2 appendix E [8], but using the QED splitting functions for $e \rightarrow \gamma e$ and $\gamma \rightarrow e \bar{e}$ appropriate to figure 7a, we find the results 
shown in (A.22). ${ }^{38}$ Note that

$$
\bar{\gamma}=-\bar{\alpha}
$$

One may check that

$$
\bar{\alpha}+\frac{1}{2} \bar{\beta}+\frac{1}{2} \bar{\gamma}=\frac{P_{e \rightarrow e}\left(x_{e}\right)}{x_{e}^{2}\left(1-x_{e}\right)^{2}} \frac{P_{\gamma \rightarrow e}\left(\frac{y_{e}}{1-x_{e}}\right)}{\left(1-x_{e}\right) y_{e}^{2}\left(1-x_{e}-y_{e}\right)^{2}},
$$

which is the straight-forward translation to QED of a similar relation for $g \rightarrow g g g .{ }^{39}$

\section{E.2 Frequencies $\Omega$ and eigenmodes}

For all of the $e \rightarrow e \bar{e} e$ diagrams of figure 7 , we will need the relevant frequencies $\Omega$ for 3-particle and 4-particle evolution, and the eigenmodes for 4-particle evolution.

\section{E.2.1 3-particle evolution frequency}

Quite generally, 3-particle frequencies are given by

$$
\Omega\left(x_{1}, x_{2}, x_{3}\right)=\sqrt{\frac{-i}{2 E}\left(\frac{\hat{q}_{1}}{x_{1}}+\frac{\hat{q}_{2}}{x_{2}}+\frac{\hat{q}_{3}}{x_{3}}\right)} .
$$

(See, for example, the review leading up to AI1 eq. (2.33b) [7].) For QED, the $\hat{q}$ of a photon is zero, and so this formula becomes

$$
\Omega\left(x_{1}^{(\bar{e})}, x_{2}^{(\gamma)}, x_{3}^{(e)}\right)=\sqrt{\frac{-i \hat{q}}{2 E}\left(\frac{1}{x_{1}^{(\bar{e})}}+\frac{1}{x_{3}^{(e)}}\right)} .
$$

For the initial 3-particle evolution of the sequential diagrams of figure $7(\mathrm{a}-\mathrm{c})$ and virtual diagrams of figure $8(\mathrm{~h}-\mathrm{k})$, this gives

$$
\Omega_{\mathrm{i}}=\sqrt{\frac{-i \hat{q}}{2 E}\left(-1+\frac{1}{x_{e}}\right)}=\sqrt{-\frac{i\left(1-x_{e}\right) \hat{q}}{2 x_{e} E}},
$$

which is equivalent to (A.24) ${ }^{40}$ and is also the frequency we quoted for $x y y \bar{x}$ in (4.12). For the sequential diagrams, the corresponding final 3-particle evolution has frequency

$$
\Omega_{\mathrm{f}}^{\mathrm{seq}}=\sqrt{\frac{-i \hat{q}}{2 E}\left(\frac{1}{y_{e}}+\frac{1}{1-x_{e}-y_{e}}\right)}=\sqrt{-\frac{i\left(1-x_{e}\right) \hat{q}}{2 y_{e}\left(1-x_{e}-y_{e}\right) E}}
$$

\footnotetext{
${ }^{38}$ Specifically, (A.22) is the $e \rightarrow e \bar{e} e$ analog of ACI2 eq. (E4) [8]. The use of the letter $z$ here is unrelated to the use in (D.3). The absolute value signs on $\left|x_{e} y_{e} z_{e}\right|$ may seem redundant here, but they are included for a reason similar to footnote 24 : to make sure that $(\bar{\alpha}, \bar{\beta}, \bar{\gamma})$ behave appropriately under front-end transformations (4.2). With the absolute value signs, a front-end transformation maps $(\bar{\alpha}, \bar{\beta}, \bar{\gamma})$ into $(1-$ $\left.x_{e}\right)^{10}$ times the analogous helicity-averaged product of $\mathcal{P}$ 's that one would have constructed for the last diagram of figure 26.

${ }^{39}$ See ACI2 eq. (E5) [8]. This is a re-assuring check because it has to hold in order for sequential diagrams to match up with sequential "Monte Carlo" when the two splittings are far separated in time. That requirement is implied by ACI2 footnote 28 [8] and the need for ACI2 eqs. (C7) and (C13) to match up accordingly. Analogous statements must hold for QED.

${ }^{40}$ We should clarify that the electron line that is called " $x_{3}^{(e) "}$ " in the context of the 3-particle expression (E.8) happens to be called " $x_{4}$ " in the context of our 4-particle variables $\left(x_{1}, x_{2}, x_{3}, x_{4}\right)$ used in the context of (A.24).
} 
for figures $7(\mathrm{a}, \mathrm{b})$ and its complex conjugate $\left(\Omega_{\mathrm{f}}^{\mathrm{seq}}\right)^{*}$ for figure $7(\mathrm{c})$. Eq. (E.10) is equivalent to (A.25) in the case $x_{i}=\hat{x}_{i}$ relevant to sequential diagrams, as in (A.14).

\section{E.2.2 4-particle evolution frequencies and modes}

For 4-particle evolution, one just needs to repeat the derivation of AI1 section V.B [7], which studied the medium-averaged evolution of four high-energy gluons in (large- $N_{\mathrm{c}}$ ) QCD. One can see from the diagrams of figures 7-9 that, for large- $N_{\mathrm{f}} \mathrm{QED}$, the only intermediate 4particles states are $\bar{e} e \bar{e} e$, and so that is the only case we address here. (Beyond the large- $N_{\mathrm{f}}$ limit, one would need to also consider $\bar{e} \gamma e \gamma$.) The only difference in the derivation is that the potential for four large- $N_{\mathrm{c}}$ gluons [AI1 eq. (4.19) [7]] is replaced by the potential

$$
V=-\frac{i \hat{q}}{4}\left[b_{12}^{2}+b_{23}^{2}+b_{34}^{2}+b_{41}^{2}-b_{13}^{2}-b_{24}^{2}\right]
$$

for $(\bar{e}, e, \bar{e}, e)$, where $\boldsymbol{b}_{i j} \equiv \boldsymbol{b}_{i}-\boldsymbol{b}_{j}$ and where the signs in front of the terms above are minus the product of the corresponding charges \pm 1 . (E.11) is algebraically equivalent to

$$
V=-\frac{i \hat{q}}{4}\left(\boldsymbol{b}_{1}-\boldsymbol{b}_{2}+\boldsymbol{b}_{3}-\boldsymbol{b}_{4}\right)^{2}
$$

Proceeding as in AI1 [7], one finds the normal mode frequencies (A.26) for $\Omega_{+}$and

$$
\Omega_{-}=0 .
$$

The corresponding eigenvectors $\vec{C}^{ \pm}$are given by (A.27) for $a_{\mathrm{y}}$ (E.3) and have been appropriately normalized so that

$$
\left(\begin{array}{l}
C_{41}^{j} \\
C_{23}^{j}
\end{array}\right)^{\top} \mathfrak{M}^{\prime}\left(\begin{array}{l}
C_{41}^{j^{\prime}} \\
C_{23}^{j^{\prime}}
\end{array}\right)=\delta^{j j^{\prime}}
$$

with

$$
\mathfrak{M}^{\prime}=\left(\begin{array}{ll}
x_{4} x_{1}\left(x_{4}+x_{1}\right) & \\
& x_{2} x_{3}\left(x_{2}+x_{3}\right)
\end{array}\right) E=\left(\begin{array}{ll}
x_{1} x_{4} & \\
& -x_{2} x_{3}
\end{array}\right)\left(x_{1}+x_{4}\right) E .
$$

We've chosen to work in the basis $\left(\boldsymbol{C}_{41}, \boldsymbol{C}_{23}\right)$ here, rather than the basis $\left(\boldsymbol{C}_{34}, \boldsymbol{C}_{12}\right)$ used in AI1 [7], in order to match the numbering used on sequential diagrams in ACI2 figure $24[8]{ }^{41}$ One may check that

$$
a_{\mathrm{y}} a_{\mathrm{y}}^{\top}=\left(\mathfrak{M}^{\prime}\right)^{-1}
$$

as implied by the normalization condition (E.14).

\section{E.3 Diagrams with instantaneous vertices}

The calculation of the real double-spitting diagrams involving instantaneous vertices, shown in figure $7(\mathrm{~d}-\mathrm{g})$, is very similar to the calculation of the QCD diagrams involving 4-point gluon vertices, shown in figure 40, which were computed in ACI4 [10].

\footnotetext{
${ }^{41}$ One may convert to the basis $\left(C_{34}, C_{12}\right)$ by simple permutation of the indices. That's because of charge conjugation symmetry, which means that the result for $(\bar{e}, e, \bar{e}, e)$ is the same as that for $(e, \bar{e}, e, \bar{e})$, and then cyclically permute the indices of the latter to get back to $(\bar{e}, e, \bar{e}, e)$ with $\left(x_{1}, x_{2}, x_{3}, x_{4}\right) \rightarrow\left(x_{2}, x_{3}, x_{4}, x_{1}\right)$.
} 

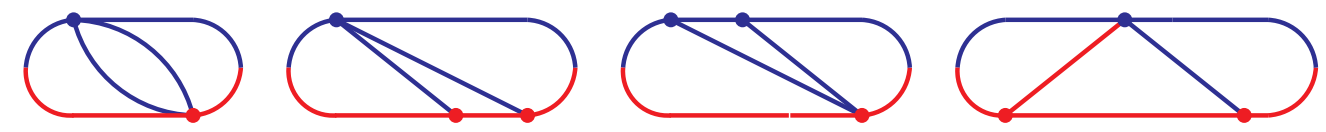

Figure 40. QCD diagrams involving 4-gluon vertices, evaluated (for large $N_{\mathrm{c}}$ ) in ACI4 [10]. In this figure, the solid lines above all represent gluons. As usual, the complex conjugates of the above interference diagrams should also be included by taking $2 \operatorname{Re}[\cdots]$ of the above.

\section{E.3.1 The $I \bar{I}$ diagram}

Consider figure $41(\mathrm{~d})$, which is a labeled version of figure $7(\mathrm{~d})$. We will call this the $I \bar{I}$ diagram with " $I$ " short for "instantaneous 4-fermion vertex." Its evaluation closely parallels that of the $4 \overline{4}$ gluon diagram of ACI4 section III.C [10], with the only change being that the overall factor

$$
\begin{aligned}
S \equiv \frac{1}{2 d_{\mathrm{A}}} \sum_{h^{\prime} \mathrm{s}} \sum_{\text {color }} & {\left[f^{a_{\mathrm{i}} a_{\mathrm{x}} e} f^{a_{\mathrm{y}} a_{\mathrm{z}} e}\left(\delta_{h_{\mathrm{i}}, h_{\mathrm{y}}} \delta_{h_{\mathrm{z}},-h_{\mathrm{x}}}-\delta_{h_{\mathrm{i}}, h_{\mathrm{z}}} \delta_{h_{\mathrm{x}},-h_{\mathrm{y}}}\right)\right.} \\
+ & f^{a_{\mathrm{i}} a_{\mathrm{y}} e} f^{a_{\mathrm{x}} a_{\mathrm{z}} e}\left(\delta_{h_{\mathrm{i}}, h_{\mathrm{x}}} \delta_{h_{\mathrm{y}},-h_{\mathrm{z}}}-\delta_{h_{\mathrm{i}}, h_{\mathrm{z}}} \delta_{h_{\mathrm{x}},-h_{\mathrm{y}}}\right) \\
+ & \left.f^{a_{\mathrm{i}} a_{\mathrm{z}} e} f^{a_{\mathrm{x}} a_{\mathrm{y}} e}\left(\delta_{h_{\mathrm{i}}, h_{\mathrm{x}}} \delta_{h_{\mathrm{y}},-h_{\mathrm{z}}}-\delta_{h_{\mathrm{i}}, h_{\mathrm{y}}} \delta_{h_{\mathrm{z}},-h_{\mathrm{x}}}\right)\right]^{2}=9 C_{\mathrm{A}}^{2}
\end{aligned}
$$

coming from the two 4-gluon vertices in ACI4 eq. (3.12) [10] is replaced here by the factor

$$
S \equiv \frac{N_{\mathrm{f}}}{2} \sum_{h^{\prime} \mathrm{s}}\left[\frac{4\left|x_{1} x_{2} x_{3} x_{4}\right|^{1 / 2}}{\left|x_{1}+x_{4}\right|^{2}} \delta_{h_{\mathrm{i}}, h_{x}} \delta_{h_{y},-h_{z}}\right]^{2}=\frac{32 N_{\mathrm{f}}\left|x_{1} x_{2} x_{3} x_{4}\right|}{\left|x_{1}+x_{4}\right|^{4}} .
$$

To see this, compare the 4-gluon vertex rule of ACI4 figure 10 [10] with the rule of figure 39 here. From ACI4 eq. (3.16) [10] (times 3 to sum up the equal results from all three color routings), the QCD gluon result was

$$
\left[\frac{d \Gamma}{d x_{e} d y_{e}}\right]_{4 \overline{4}}=-\frac{9 C_{\mathrm{A}}^{2} \alpha_{\mathrm{s}}^{2}}{16 \pi^{2}} \int_{0}^{\infty} d(\Delta t) \Omega_{+} \Omega_{-} \csc \left(\Omega_{+} \Delta t\right) \csc \left(\Omega_{-} \Delta t\right)
$$

The corresponding result here is then

$$
\begin{aligned}
{\left[\frac{d \Gamma}{d x_{e} d y_{e}}\right]_{I \bar{I}} } & =-\frac{2 N_{\mathrm{f}} \alpha_{\mathrm{EM}}^{2}}{\pi^{2}} \frac{x_{e} y_{e} z_{e}}{\left(1-x_{e}\right)^{4}} \int_{0}^{\infty} d(\Delta t) \Omega_{+} \Omega_{-} \csc \left(\Omega_{+} \Delta t\right) \csc \left(\Omega_{-} \Delta t\right) \\
& =-\frac{2 N_{\mathrm{f}} \alpha_{\mathrm{EM}}^{2}}{\pi^{2}} \frac{x_{e} y_{e} z_{e}}{\left(1-x_{e}\right)^{4}} \int_{0}^{\infty} \frac{d(\Delta t)}{\Delta t} \Omega_{+} \csc \left(\Omega_{+} \Delta t\right) .
\end{aligned}
$$

Following our general procedure from AI1 [7] of subtracting out the vacuum pieces of each diagram (which must all cancel in the final result), this is

$$
\begin{aligned}
{\left[\frac{d \Gamma}{d x_{e} d y_{e}}\right]_{I \bar{I}} } & =-\frac{2 N_{\mathrm{f}} \alpha_{\mathrm{EM}}^{2}}{\pi^{2}} \frac{x_{e} y_{e} z_{e}}{\left(1-x_{e}\right)^{4}} \int_{0}^{\infty} \frac{d(\Delta t)}{\Delta t}\left[\Omega_{+} \csc \left(\Omega_{+} \Delta t\right)-\frac{1}{\Delta t}\right] \\
& =\frac{2 N_{\mathrm{f}} \alpha_{\mathrm{EM}}^{2}}{\pi^{2}} \frac{x_{e} y_{e} z_{e}}{\left(1-x_{e}\right)^{4}} i \Omega_{+} \ln 2 .
\end{aligned}
$$

Adding this diagram to its complex conjugate gives what we labeled as the "(II)" contribution (A.35) to the total answer (A.11). 


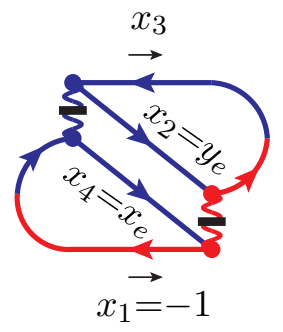

(d)

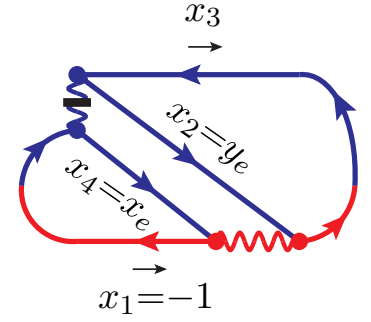

(e)

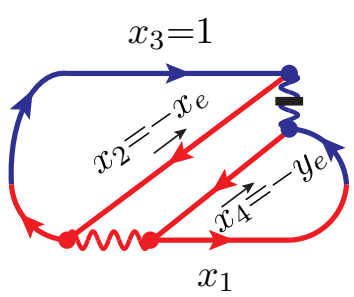

(f)

Figure 41. Labeling of lines for the (d) $I \bar{I}$, (e) $I \bar{x} \bar{y}$, and (f) $\bar{x} \bar{y} I$ diagrams of figures $7(\mathrm{~d}-\mathrm{f})$.

\section{E.3.2 Diagrams with one instantaneous vertex}

Now consider the interference diagram of figure $41(\mathrm{e})$. Remember that our (large- $N_{\mathrm{f}}$ ) convention is that $x_{e}$ is the momentum fraction of the particular final-state electron that is connected by an electron line to the initial electron.

There are just a few differences with the similar calculation of the $4 \bar{x} \bar{y}$ gluon process given in ACI4 section II [10]. First, comparing figure 41(e) here with ACI4 figure 12(a), our labeling conventions are a little different. The translation is that $\left(\hat{x}_{1}, \hat{x}_{2}, \hat{x}_{3}, \hat{x}_{4}\right)=$ $(-1, y, 1-x-y, x)$ in ref. [10] is permuted to $\left(\hat{x}_{1}, \hat{x}_{4}, \hat{x}_{2}, \hat{x}_{3}\right)=\left(-1, x_{e}, y_{e}, 1-x_{e}-y_{e}\right)$ here. Another is that the 4-point vertex factor

$$
\begin{aligned}
& f^{a_{\mathrm{i}} a_{\mathrm{x}} e} f^{a_{\mathrm{y}} a_{\mathrm{z}} e}\left(\delta_{h_{\mathrm{i}}, h_{\mathrm{y}}} \delta_{h_{\mathrm{z}},-h_{\mathrm{x}}}-\delta_{h_{\mathrm{i}}, h_{\mathrm{z}}} \delta_{h_{\mathrm{x}},-h_{\mathrm{y}}}\right) \\
& \quad+\frac{1}{2} f^{a_{\mathrm{i}} a_{\mathrm{y}} e} f^{a_{\mathrm{x}} a_{\mathrm{z}} e}\left(\delta_{h_{\mathrm{i}}, h_{\mathrm{x}}} \delta_{h_{\mathrm{y}},-h_{\mathrm{z}}}-\delta_{h_{\mathrm{i}}, h_{\mathrm{z}}} \delta_{h_{\mathrm{x}},-h_{\mathrm{y}}}\right)
\end{aligned}
$$

of ACI4 eq. (2.9) for its color routing $4 \bar{y} \bar{x}_{2}$ should be replaced by

$$
\frac{4\left|x_{1} x_{2} x_{3} x_{4}\right|^{1 / 2}}{\left(x_{2}+x_{3}\right)^{2}} \delta_{h_{\mathrm{i}}, h_{\mathrm{x}}} \delta_{h_{\mathrm{y}},-h_{\mathrm{z}}}=\frac{4\left(x_{e} y_{e} z_{e}\right)^{1 / 2}}{\left(1-x_{e}\right)^{2}} \delta_{h_{\mathrm{i}}, h_{\mathrm{x}}} \delta_{h_{\mathrm{y}},-h_{\mathrm{z}}}
$$

for the instantaneous vertex in our calculation here, for which there are no color routings to consider. (Again, compare the 4-gluon vertex rule of ACI4 figure 10 [10] with the rule of figure 39 here.) Also, unlike ACI4 eq. (2.10), there are no color factors associated with the other vertices. Finally, $g_{\mathrm{s}}^{4}$ becomes $N_{\mathrm{f}} g_{\mathrm{EM}}^{4}$. Making these changes, and keeping track of the signs of the $\boldsymbol{B}_{i j}$ in our figure 37 rule, we find that ACI4 eq. (2.12) translates to

$$
\begin{aligned}
& {\left[\frac{d I}{d x d y}\right]_{I \bar{x} \bar{y}}=-\left(\frac{E}{2 \pi}\right)^{2} \int_{t_{(4)}<t_{\bar{y}}<t_{\overline{\mathrm{x}}}} \sum_{h_{\mathrm{x}}, h_{\mathrm{y}}, h_{z}, \bar{h}} \int_{B^{\overline{\mathrm{x}}}}} \\
& \times \frac{4 i\left(x_{e} y_{e} z_{e}\right)^{1 / 2}}{\left(1-x_{e}\right)^{2}} N_{\mathrm{f}} g^{4} \delta_{h_{\mathrm{i}}, h_{\mathrm{x}}} \delta_{h_{\mathrm{y}},-h_{\mathrm{z}}}
\end{aligned}
$$

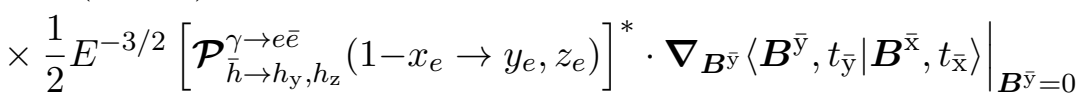

$$
\begin{aligned}
& \times \frac{1}{2} E^{-3 / 2}\left|\hat{x}_{2}+\hat{x}_{3}\right|^{-1}\left[\mathcal{P}_{h_{\mathrm{i}} \rightarrow \bar{h}, h_{\mathrm{x}}}^{e \rightarrow \gamma}\left(1 \rightarrow 1-x_{e}, x_{e}\right)\right]^{*} \cdot \nabla_{\boldsymbol{C}_{14}^{\overline{\mathrm{x}}}} \\
& \left.\left\langle\boldsymbol{C}_{23}^{\overline{\mathrm{x}}}, \boldsymbol{C}_{14}^{\overline{\mathrm{x}}}, t_{\overline{\mathrm{x}}} \mid \boldsymbol{C}_{23}^{I}, \boldsymbol{C}_{14}^{I}, t_{I}\right\rangle\right|_{\boldsymbol{C}_{14}^{\overline{\mathrm{x}}}=0=\boldsymbol{C}_{14}^{I}=\boldsymbol{C}_{23}^{I} ; \boldsymbol{C}_{23}^{\overline{\mathrm{x}}}=\boldsymbol{B}^{\overline{\mathrm{x}}}} \\
& \times(2 E)^{-2}\left|\hat{x}_{1} \hat{x}_{2} \hat{x}_{3} \hat{x}_{4}\right|^{-1 / 2}\left|\hat{x}_{2}+\hat{x}_{3}\right|^{-1} \text {. }
\end{aligned}
$$


The helicity sum analogous to ACI4 eqs. (2.14) is

$$
\begin{aligned}
\sum_{h_{\mathrm{x}}, h_{\mathrm{y}}, h_{\mathrm{z}}} & {\left[\sum_{\bar{h}}\left[\mathcal{P}_{\bar{h} \rightarrow h_{\mathrm{y}}, h_{\mathrm{z}}}^{\gamma \rightarrow \overline{\mathrm{z}}}\right]^{\bar{n}}\left(1-x_{e} \rightarrow y_{e}, z_{e}\right)\left[\mathcal{P}_{h_{\mathrm{i}} \rightarrow \bar{h}, h_{\mathrm{x}}}^{e \rightarrow \gamma e}\right]^{\bar{m}}\left(1 \rightarrow 1-x_{e}, x_{e}\right)\right]^{*} } \\
& \times \delta_{h_{\mathrm{i}}, h_{\mathrm{x}}} \delta_{h_{\mathrm{y}},-h_{\mathrm{z}}}\left|\hat{x}_{1} \hat{x}_{2} \hat{x}_{3} \hat{x}_{4}\right|^{-1 / 2} .
\end{aligned}
$$

Using the QED formulas in appendix D here, we find that the initial-helicity average of (E.25) above is

$$
\zeta\left(x_{e}, y_{e}\right) \delta^{\bar{n} \bar{m}}
$$

with $^{42}$

$$
\zeta=\frac{\left(1+\left|x_{e}\right|\right)\left(\left|y_{e}\right|+\left|z_{e}\right|\right)}{\left(1-x_{e}\right)^{3}\left(x_{e} y_{e} z_{e}\right)^{3 / 2}}
$$

Contrast to ACI4 eq. (2.16). Following ACI4, the final result, analogous to ACI4 eq. (2.25), is then

$$
\begin{aligned}
{\left[\frac{d \Gamma}{d x d y}\right]_{I \bar{x} \bar{y}}=} & -\frac{N_{\mathrm{f}} \alpha_{\mathrm{EM}}^{2} M_{\mathrm{f}}^{\mathrm{seq}}}{2 \pi^{2} E} \frac{\left(x_{e} y_{e} z_{e}\right)^{1 / 2}}{\left(1-x_{e}\right)^{2}}\left(-\hat{x}_{1} \hat{x}_{2} \hat{x}_{3} \hat{x}_{4}\right) \zeta \\
& \times \int_{0}^{\infty} d(\Delta t) \Omega_{+} \Omega_{-} \csc \left(\Omega_{+} \Delta t\right) \csc \left(\Omega_{-} \Delta t\right) \frac{Y_{\overline{\mathrm{x}}}^{\mathrm{seq}}}{X_{\overline{\mathrm{x}}}^{\mathrm{seq}}} \\
= & -\frac{N_{\mathrm{f}} \alpha_{\mathrm{EM}}^{2}}{2 \pi^{2}} \frac{\left(1+\left|x_{e}\right|\right) y_{e} z_{e}\left(\left|y_{e}\right|+\left|z_{e}\right|\right)}{\left(1-x_{e}\right)^{4}} \int_{0}^{\infty} \frac{d(\Delta t)}{\Delta t} \Omega_{+} \csc \left(\Omega_{+} \Delta t\right) \frac{Y_{\overline{\mathrm{x}}}^{\mathrm{seq}}}{X_{\overline{\mathrm{x}}}^{\mathrm{seq}}}
\end{aligned}
$$

where $X_{\overline{\mathrm{x}}}^{\text {seq }}$ and $Y_{\overline{\mathrm{x}}}^{\mathrm{seq}}$ are as in (A.21).

Following the strategy of ACI4 section III.A, the $\bar{x} \bar{y} I$ diagram of figure $41(\mathrm{f})$ here is like the mirror reflection of $41(\mathrm{e})$. Given how we have labeled particles, ${ }^{43}$ this mirror reflection transformation can be achieved by the replacement

$$
\left(x_{1}, x_{2}, x_{3}, x_{4}\right) \rightarrow\left(-\hat{x}_{3},-\hat{x}_{4},-\hat{x}_{1},-\hat{x}_{2}\right),
$$

including inside of $M_{\mathrm{f}}^{\mathrm{seq}}=x_{2} x_{3}\left(x_{2}+x_{3}\right) E$ and

$$
\Omega_{\mathrm{f}}^{\mathrm{seq}}\left(x_{1}, x_{2}, x_{3}, x_{4}\right)=\sqrt{\frac{-i \hat{q}}{2 E}\left(\frac{1}{x_{2}}+\frac{1}{x_{3}}\right)} .
$$

For the $I \bar{x} \bar{y}$ diagram, $\Omega_{\mathrm{f}}^{\text {seq }}\left(\hat{x}_{1}, \hat{x}_{2}, \hat{x}_{3}, \hat{x}_{4}\right)$ is just a way to write the $\Omega_{\mathrm{f}}^{\text {seq }}$ of (E.10) in terms of the 4-particle variables $\left(\hat{x}_{1}, \hat{x}_{2}, \hat{x}_{3}, \hat{x}_{4}\right)$. But the form (E.30) has the advantage that, after the substitution (E.29), it also gives the correct $\Omega$ for the initial 3-particle evolution for $\bar{x} \bar{y} I$ as depicted in figure $41(\mathrm{f})$.

\footnotetext{
${ }^{42}$ Similar to the discussion in footnote 38 , absolute value signs have been judiciously included in (A.30) so that $\zeta$ does the right thing under front-end transformations, which for the diagram at hand is implemented by (4.5). Note that the front-end transformation rule (4.5) for this diagram does not change the sign of $x_{e} y_{e} z_{e}$, and so there is no need to write $\left|x_{e} y_{e} z_{e}\right|$ here like there was in (A.22).

${ }^{43}$ The labeling of figures $41(\mathrm{e})$ and (f), and so the specifics of the transformation (E.29), are slightly different than in ACI4 in order to maintain our convention here that $x_{e}$ always refers to the final-state electron whose electron line is connected to the initial-state electron.
} 
The $\bar{x} I \bar{y}$ diagram of figure $7(\mathrm{~g})$ vanishes by parity symmetry for the same reasons as the $\bar{y} 4 \bar{x}$ diagram in ACI4 section III.B.

These results for diagrams with one instantaneous vertex are summarized in section A.2.2.

\section{F Explicit formulas for evaluation of $x y y \bar{x}$ diagram}

\section{F.1 General formula for $d=2$}

From the starting formula (4.8), following the same steps as our earlier work $[7,8]$ $(\mathrm{AI} 1, \mathrm{ACI} 2)$ on real double splitting rates in (large- $N_{\mathrm{c}}$ ) QCD, one obtains formulas that schematically have the same form. Namely,

$$
\begin{aligned}
{\left[\frac{d \Gamma}{d x_{e} d y_{e}}\right]_{x y y \bar{x}}=-\frac{N_{\mathrm{f}} \alpha_{\mathrm{EM}}^{2} M_{\mathrm{i}}^{2}}{16 \pi^{4} E^{2}}(} & \left.-\hat{x}_{1} \hat{x}_{2} \hat{x}_{3} \hat{x}_{4}\right) \int_{0}^{\infty} d(\Delta t) \Omega_{+} \Omega_{-} \csc \left(\Omega_{+} \Delta t\right) \csc \left(\Omega_{-} \Delta t\right) \\
\times & \left\{\left(\bar{\beta} Y_{\mathrm{y}}^{2}+\bar{\gamma} \bar{Y}_{\mathrm{yy}^{\prime}} Y_{\mathrm{yy}^{\prime}}\right) I_{0}^{\text {new }}+(2 \bar{\alpha}+\bar{\beta}+\bar{\gamma}) Z_{\mathrm{yy}} I_{1}^{\text {new }}\right. \\
& +\left[(\bar{\alpha}+\bar{\gamma}) Y_{\mathrm{y}}^{2}+(\bar{\alpha}+\bar{\beta}) \bar{Y}_{\mathrm{yy}^{\prime}} Y_{\mathrm{yy}^{\prime}}\right] I_{2}^{\text {new }} \\
& \left.-(\bar{\alpha}+\bar{\beta}+\bar{\gamma})\left(\bar{Y}_{\mathrm{yy}^{\prime}} Y_{\mathrm{y}} I_{3}^{\text {new }}+Y_{\mathrm{y}} Y_{\mathrm{yy}^{\prime}} I_{4}^{\text {new }}\right)\right\}
\end{aligned}
$$

[which here must eventually be integrated over $y_{e}$ as in (4.7)]. In our QED application, $\Omega_{-}=0$ (E.13), and so

$$
\Omega_{-} \csc \left(\Omega_{-} \Delta t\right)=\frac{1}{\Delta t}
$$

above. The $I_{n}^{\text {new }}$ are just like the $I_{n}^{\text {seq }}$ of (A.20),

$$
\begin{aligned}
I_{0}^{\text {new }} & =\frac{4 \pi^{2}}{\left[X_{\mathrm{y}}^{\text {new }} X_{\mathrm{y}^{\prime}}^{\text {new }}-\left(X_{\mathrm{yy}^{\prime}}^{\text {new }}\right)^{2}\right]} \\
I_{1}^{\text {new }} & =-\frac{2 \pi^{2}}{X_{\mathrm{yy}}^{\text {new }}} \ln \left(1-\frac{\left(X_{\mathrm{yy}^{\prime}}^{\text {new }}\right)^{2}}{X_{\mathrm{y}}^{\text {new }} X_{\mathrm{y}^{\prime}}^{\text {new }}}\right) \\
I_{2}^{\text {new }} & =\frac{2 \pi^{2}}{\left(X_{\mathrm{yy}}^{\text {new }}\right)^{2}} \ln \left(1-\frac{\left(X_{\mathrm{yy}^{\prime}}^{\text {new }}\right)^{2}}{X_{\mathrm{y}}^{\text {new }} X_{\mathrm{y}^{\prime}}^{\text {new }}}\right)+\frac{4 \pi^{2}}{\left[X_{\mathrm{y}}^{\text {new }} X_{\mathrm{y}^{\prime}}^{\text {new }}-\left(X_{\mathrm{yy}}^{\text {new }}\right)^{2}\right]} \\
I_{3}^{\text {new }} & =\frac{4 \pi^{2} X_{\mathrm{yy}^{\prime}}^{\text {new }}}{X_{\mathrm{y}^{\prime}}^{\text {new }}\left[X_{\mathrm{y}}^{\text {new }} X_{\mathrm{y}^{\prime}}^{\text {new }}-\left(X_{\mathrm{yy}^{\prime}}^{\text {new }}\right)^{2}\right]} \\
I_{4}^{\text {new }} & =\frac{4 \pi^{2} X_{\mathrm{yy}^{\prime}}^{\text {new }}}{X_{\mathrm{y}}^{\text {new }}\left[X_{\mathrm{y}}^{\text {new }} X_{\mathrm{y}^{\prime}}^{\text {new }}-\left(X_{\mathrm{yy}^{\prime}}^{\text {new }}\right)^{2}\right]}
\end{aligned}
$$

except that the $(X, Y, Z)^{\text {seq }}$ of (A.21) are replaced by $(X, Y, Z)^{\text {new }}$ in which $M_{\mathrm{f}}^{\text {seq }}$ is replaced by $M_{\mathrm{i}}$ (because the final 3-particle evolution in the $x y y \bar{x}$ diagram, figure 29 , involves the same particles as the initial stage of 3 -particle evolution) and the $a_{\overline{\mathrm{x}}}^{\text {seq }}$ are replaced by $a_{\mathrm{y}^{\prime}}=a_{\mathrm{y}}$ (because the particles that merge at the end of the interim 4-particle evolution 
are the same as the ones that split at its start):

$$
\begin{aligned}
\left(\begin{array}{cc}
X_{\mathrm{y}}^{\text {new }} & Y_{\mathrm{y}}^{\text {new }} \\
Y_{\mathrm{y}}^{\text {new }} & Z_{\mathrm{y}}^{\text {new }}
\end{array}\right)= & \left(\begin{array}{ll}
X_{\mathrm{y}^{\prime}}^{\text {new }} & Y_{\mathrm{y}^{\prime}}^{\text {new }} \\
Y_{\mathrm{y}^{\prime}}^{\text {new }} & Z_{\mathrm{y}^{\prime}}^{\text {new }}
\end{array}\right) \equiv\left(\begin{array}{cc}
\left|M_{\mathrm{i}}\right| \Omega_{\mathrm{i}} & 0 \\
0 & 0
\end{array}\right)-i a_{\mathrm{y}}^{-1 \top}\left(\begin{array}{cc}
\Omega_{+} \cot \left(\Omega_{+} \Delta t\right) & 0 \\
0 & (\Delta t)^{-1}
\end{array}\right) a_{\mathrm{y}}^{-1}, \\
& \left(\begin{array}{cc}
X_{\mathrm{yy}}^{\text {new }} & Y_{\mathrm{yy}}^{\text {new }} \\
\bar{Y}_{\mathrm{yy}^{\prime}}^{\text {new }} & Z_{\mathrm{yy}^{\prime}}^{\text {new }}
\end{array}\right) \equiv-i a_{\mathrm{y}}^{-1 \top}\left(\begin{array}{cc}
\Omega_{+} \csc \left(\Omega_{+} \Delta t\right) & 0 \\
0 & (\Delta t)^{-1}
\end{array}\right) a_{\mathrm{y}}^{-1} \cdot \quad(\mathrm{F} .4 \mathrm{~b})
\end{aligned}
$$

\section{F.2 Small $\Delta t$ expansion}

\section{F.2.1 Structure}

To study the UV divergence of $x y y \bar{x}$, we want to identify which terms of the integrand in (F.1) are important as $\Delta t \rightarrow 0$. For that, we need the small- $\Delta t$ expansion of (F.4). At leading order in $\Delta t$, the expansion is the same for $(X, Y, Z)_{\mathrm{y}}^{\text {new }}$ and $(X, Y, Z)_{\mathrm{yy}^{\prime}}^{\text {new }}$ :

$$
\left(\begin{array}{ll}
X^{\text {new }} & Y^{\text {new }} \\
Y^{\text {new }} & Z^{\text {new }}
\end{array}\right) \simeq-i \frac{a_{\mathrm{y}}^{-1 \top} a_{\mathrm{y}}^{-1}}{\Delta t}=\frac{-i E\left(x_{1}+x_{4}\right)}{\Delta t}\left(\begin{array}{cc}
x_{1} x_{4} & 0 \\
0 & -x_{2} x_{3}
\end{array}\right),
$$

where the second equality uses (E.15) and (E.16). This approximation is fine for the $Y$ 's and $Z$ 's in (F.1), but it is inadequate for the combination $X_{\mathrm{y}}^{\text {new }} X_{\mathrm{y}^{\prime}}^{\text {new }}-\left(X_{\mathrm{yy}^{\prime}}^{\text {new }}\right)^{2}$ that appears in (F.3), which is zero at the order of (F.5).

Going to next order in $\Delta t$,

$$
\begin{aligned}
X_{\mathrm{y}}^{\text {new }}=X_{\mathrm{y}^{\prime}}^{\text {new }} & \simeq \frac{-i M_{\mathrm{i}}}{\Delta t}+\left|M_{\mathrm{i}}\right| \Omega_{\mathrm{i}}+O(\Delta t), \\
X_{\mathrm{yy}^{\prime}}^{\text {new }} & \simeq \frac{-i M_{\mathrm{i}}}{\Delta t}+O(\Delta t), \\
Y_{\text {any }}^{\text {new }} & =O(\Delta t), \\
Z_{\text {any }}^{\text {new }} & =\frac{i x_{2} x_{3}\left(x_{1}+x_{4}\right) E}{\Delta t}+O(\Delta t),
\end{aligned}
$$

which gives

$$
X_{\mathrm{y}}^{\text {new }} X_{\mathrm{y}^{\prime}}^{\text {new }}-\left(X_{\mathrm{yy}^{\prime}}^{\text {new }}\right)^{2}=\frac{-2 i M_{\mathrm{i}}^{2} \Omega_{\mathrm{i}}}{\Delta t} \operatorname{sgn} M_{\mathrm{i}}+O\left((\Delta t)^{0}\right) .
$$

One then finds that the $I_{n}^{\text {new }}$ of (F.3) are all $O(\Delta t)$. Using these expansions in (F.1) shows that all the terms in the integrand are finite as $\Delta t \rightarrow 0$ except for the term involving $Z_{\mathrm{yy}} I_{1}$, whose dependence on $\Delta t$ is

$$
\Omega_{+} \Omega_{-} \csc \left(\Omega_{+} \Delta t\right) \csc \left(\Omega_{-} \Delta t\right) Z_{\mathrm{yy}} I_{1}^{\text {new }} \sim \frac{1}{(\Delta t)^{2}} .
$$

This $Z_{\mathrm{yy}} I_{1}$ term contains the small- $\Delta t$ divergence represented by (4.25) in the main text. [We will discuss the translation to (4.25) later.]

Because (F.7) blows up as $(\Delta t)^{-2}$, we also potentially have a sub-leading divergence $(\Delta t)^{-1}$ if there are any corrections in the small- $\Delta t$ expansion that are suppressed by only one more power of $\Delta t$. Tracing back through (F.7) and the expansions (F.6), and noting 
that $\ln \left[1-X_{\mathrm{yy}^{\prime}}^{2} / X_{\mathrm{y}} X_{\mathrm{y}^{\prime}}\right]=\ln \left[\left(X_{\mathrm{y}} X_{\mathrm{y}^{\prime}}-X_{\mathrm{yy}^{\prime}}^{2}\right) / X_{\mathrm{y}} X_{\mathrm{y}^{\prime}}\right]$ in (F.3b), the only correction that contributes at this order is the $O\left((\Delta t)^{0}\right)$ correction to (F.6e). Computing this correction requires the $O(\Delta t)$ terms in (F.6a) and (F.6b).

There is a simplification that will allow us to work out a useful formula for the needed corrections to $X_{\mathrm{y}}^{\text {new }}$ and $X_{\mathrm{y}^{\prime}}^{\text {new }}$ more generally than for the QED application of relevance here. So, even though $\Omega_{-}=0$ for QED, let us be more general and rewrite (F.4) as

$$
\begin{aligned}
\left(\begin{array}{cc}
X_{\mathrm{y}}^{\text {new }} & Y_{\mathrm{y}}^{\text {new }} \\
Y_{\mathrm{y}}^{\text {new }} & Z_{\mathrm{y}}^{\text {new }}
\end{array}\right)= & \left(\begin{array}{cc}
X_{\mathrm{y}^{\prime}}^{\text {new }} & Y_{\mathrm{y}^{\prime}}^{\text {new }} \\
Y_{\mathrm{y}^{\prime}}^{\text {new }} & Z_{\mathrm{y}^{\prime}}^{\text {new }}
\end{array}\right) \equiv\left(\begin{array}{cc}
\left|M_{\mathrm{i}}\right| \Omega_{\mathrm{i}} & 0 \\
0 & 0
\end{array}\right)-i a_{\mathrm{y}}^{-1 \top} \underline{\Omega} \cot (\underline{\Omega} \Delta t) a_{\mathrm{y}}^{-1}, \\
& \left(\begin{array}{cc}
X_{\mathrm{yy}^{\prime}}^{\text {new }} & Y_{\mathrm{yy}^{\prime}}^{\text {new }} \\
\bar{Y}_{\mathrm{yy}^{\prime}}^{\text {new }} & Z_{\mathrm{yy}^{\prime}}^{\text {new }}
\end{array}\right) \equiv-i a_{\mathrm{y}}^{-1 \top} \underline{\Omega} \csc (\underline{\Omega} \Delta t) a_{\mathrm{y}}^{-1}
\end{aligned}
$$

with

$$
\underline{\Omega} \equiv\left(\begin{array}{ll}
\Omega_{+} & \\
& \Omega_{-}
\end{array}\right) .
$$

Noting that (4.9) and (A.19) give $x_{1} x_{4}\left(x_{1}+x_{4}\right) E=M_{\mathrm{i}}$ in (F.5), the small- $\Delta t$ expansions of the $X$ 's are then

$$
\begin{aligned}
X_{\mathrm{y}}^{\text {new }}=X_{\mathrm{y}^{\prime}}^{\text {new }} & =\frac{-i M_{\mathrm{i}}}{\Delta t}+\left|M_{\mathrm{i}}\right| \Omega_{\mathrm{i}}+\frac{i}{3}\left[a_{\mathrm{y}}^{-1 \top} \underline{\Omega}^{2} a_{\mathrm{y}}^{-1}\right]_{11} \Delta t+O\left((\Delta t)^{3}\right), \\
X_{\mathrm{yy}^{\prime}}^{\text {new }} & =\frac{-i M_{\mathrm{i}}}{\Delta t}-\frac{i}{6}\left[a_{\mathrm{y}}^{-1 \top} \underline{\Omega}^{2} a_{\mathrm{y}}^{-1}\right]_{11} \Delta t+O\left((\Delta t)^{3}\right) .
\end{aligned}
$$

Then

$$
X_{\mathrm{y}}^{\text {new }} X_{\mathrm{y}^{\prime}}^{\text {new }}-\left(X_{\mathrm{yy}^{\prime}}^{\text {new }}\right)^{2}=\frac{-2 i M_{\mathrm{i}}^{2} \Omega_{\mathrm{i}}}{\Delta t} \operatorname{sgn} M_{\mathrm{i}}+M_{\mathrm{i}}^{2} \Omega_{\mathrm{i}}^{2}+M_{\mathrm{i}}\left[a_{\mathrm{y}}^{-1 \top} \underline{\Omega}^{2} a_{\mathrm{y}}^{-1}\right]_{11}+O(\Delta t) .
$$

The expression $\left[a_{\mathrm{y}}^{-1 \top} \underline{\Omega}^{2} a_{\mathrm{y}}^{-1}\right]_{11}$ sounds like a mess that depends on detailed formulas for $\Omega_{ \pm}$and $a_{\mathrm{y}}$. Happily, it can be recast into a very simple form.

\section{F.2.2 Value of $a^{-1^{\top}} \Omega^{2} a^{-1}$}

As in appendix E.2.2 above, return again to the derivation of eigenfrequencies $\Omega_{ \pm}$and normal modes in AI1 section V.B [7]. From AI1 (5.17), the relevant Lagrangian has the form

$$
L=\frac{1}{2} \dot{\vec{C}}^{\top} \mathfrak{M} \dot{\vec{C}}-\frac{1}{2} \vec{C}^{\top} \mathfrak{K} \vec{C},
$$

where $\vec{C}$ is $\left(C_{41}, C_{23}\right)$ or whatever permutation you want, and $\mathfrak{M}$ and $\mathfrak{K}$ are $2 \times 2$ matrices in that basis. The $\frac{1}{2} \vec{C}^{\top} \mathfrak{K} \vec{C}$ above encodes the potential $\mathrm{V}$ of the double harmonic oscillator problem, e.g. (E.12) in our case. For our basis choice $\left(C_{41}, C_{23}\right), \mathfrak{M}$ is given by (E.15).

The equation of motion from (F.12) is

$$
\mathfrak{M} \ddot{\vec{C}}=-\mathfrak{K} \ddot{\vec{C}},
$$

and the corresponding normal-mode eigenvalue problem is

$$
\mathfrak{M} \Omega_{ \pm}^{2} \vec{C}^{ \pm}=\mathfrak{K} \vec{C}^{ \pm}
$$


We can rewrite both cases $( \pm)$ of this equation simultaneously as

$$
\mathfrak{M} a \underline{\Omega}^{2}=\mathfrak{K} a,
$$

where

$$
a \equiv\left(\vec{C}^{+} \mid \vec{C}^{-}\right)
$$

is the $2 \times 2$ matrix $a_{\mathrm{y}}$ [(E.3) in this paper, or whatever permutation is relevant to one's choice of basis for $\vec{C}]$.

Multiplying (F.15) by $a^{\top}$ on the left,

$$
a^{\top} \mathfrak{M} a \underline{\Omega}^{2}=a^{\top} \mathfrak{K} a .
$$

Because the normal modes are orthogonal with respect to $\mathcal{M}$ and then normalized (E.14) so that

$$
\left(\vec{C}^{j}\right)^{\top} \mathfrak{M} \vec{C}^{j^{\prime}}=\delta^{j j^{\prime}},
$$

we have $a^{\top} \mathfrak{M} a=\mathbb{1}$ and so

$$
\underline{\Omega}^{2}=a^{\top} \mathfrak{K} a .
$$

Thus,

$$
\left(a^{-1}\right)^{\top} \underline{\Omega}^{2} a^{-1}=\mathfrak{K} .
$$

This result is independent of details about the eigenfrequencies or eigenmodes and which basis we pick for $\vec{C}$.

In the application to $x y y \bar{x}$, we want in particular the first element of this matrix,

$$
\left[\left(a^{-1}\right)^{\top} \underline{\Omega}^{2} a^{-1}\right]_{11}=\mathfrak{K}_{11},
$$

in the $\left(C_{41}, C_{23}\right)$ basis (numbered as in the right-hand diagram of figure 29). In that context, $\mathfrak{K}_{11}$ corresponds to the (complex) spring constant of the problem if we were to set $C_{23}$ to zero. That would be the same as setting $\boldsymbol{b}_{2}=\boldsymbol{b}_{3}$ and so the same as placing the $e$ and $\bar{e}$ of the photon self-energy loop on top of each other. In that case, the $e \bar{e}$ pair is, with regard to charge, indistinguishable from the photon that created them (i.e. no charge in the QED case here). In this case, the potential in the 4-particle evolution of figure 29 would be the same as that in the preceding 3 -particle portion. But that means that $\mathfrak{K}_{11}$ must be identical to the spring constant for the initial 3-particle evolution, and so

$$
\mathfrak{K}_{11}=M_{\mathrm{i}} \Omega_{\mathrm{i}}^{2}
$$

in the context of the $x y y \bar{x}$ diagram. One may verify this general relation in our QED case by starting from the potential (E.12), following the method of AI1 section V.B [7] to construct

$$
\mathfrak{K}=-\frac{i \hat{q}}{2}\left(x_{1}+x_{4}\right)^{2}\left(\begin{array}{rr}
1 & -1 \\
-1 & 1
\end{array}\right)
$$

in the $\left(C_{41}, C_{23}\right)$ basis, and then checking (F.22) using (4.9), (4.12), and (4.22). A similar check works in the case of (large- $N_{\mathrm{c}}$ ) QCD. 


\section{F.2.3 Putting it together}

Using the results above, (F.11) becomes

$$
X_{\mathrm{y}}^{\text {new }} X_{\mathrm{y}^{\prime}}^{\text {new }}-\left(X_{\mathrm{yy}^{\prime}}^{\text {new }}\right)^{2}=\frac{-2 i M_{\mathrm{i}}^{2} \Omega_{\mathrm{i}}}{\Delta t} \operatorname{sgn} M_{\mathrm{i}}+2 M_{\mathrm{i}}^{2} \Omega_{\mathrm{i}}^{2}+O(\Delta t)
$$

Using this and our earlier expansions in (F.1) gives the unregulated $(\epsilon=0)$ divergence

$$
\left[\frac{d \Gamma}{d x_{e} d y_{e}}\right]_{x y y \bar{x}}^{(\Delta t<a)}=-\frac{N_{\mathrm{f}} \alpha_{\mathrm{EM}}^{2} M_{\mathrm{i}}^{2}}{16 \pi^{4} E^{2}}\left(-\hat{x}_{1} \hat{x}_{2} \hat{x}_{3} \hat{x}_{4}\right)(2 \bar{\alpha}+\bar{\beta}+\bar{\gamma}) \int_{0}^{a} \frac{d(\Delta t)}{(\Delta t)^{2}} Z_{\mathrm{yy}} I_{1}^{\mathrm{new}}+O(a)
$$

with

$$
\begin{aligned}
\int_{0}^{a} \frac{d(\Delta t)}{(\Delta t)^{2}} Z_{\mathrm{yy}^{\prime}} I_{1}^{\text {new }} & =\frac{\hat{x}_{2} \hat{x}_{3}}{\hat{x}_{1} \hat{x}_{4}} \mathbb{I}_{\text {unregulated }} \\
\mathbb{I}_{\text {unregulated }} & =2 \pi^{2} \int_{0}^{a} d(\Delta t)\left[\frac{\ln \left(2 i \bar{\Omega}_{\mathrm{i}} \Delta t\right)}{(\Delta t)^{2}}-\frac{i \bar{\Omega}_{\mathrm{i}}}{\Delta t}\right]+O(a) .
\end{aligned}
$$

This is the origin of (4.11) in the main text. As we will see below, the $\mathbb{I}_{\text {unregulated }}$ above is the unregulated $(\epsilon=0)$ version of the $\mathbb{I}$ introduced in (4.30) of the main text. We will see that the $\mathcal{X}$ of (4.25) turn out to represent the $X^{\text {new }}$ of (F.4) without the $\left|M_{\mathrm{i}}\right| \Omega_{\mathrm{i}}$ terms, i.e.

$$
X_{\mathrm{y}}^{\text {new }}=\left|M_{\mathrm{i}}\right| \Omega_{\mathrm{i}}+\mathcal{X}_{\mathrm{y}}, \quad X_{\mathrm{yy}^{\prime}}^{\text {new }}=\mathcal{X}_{\mathrm{yy}^{\prime}}
$$

The expansions (4.26) of the $\mathcal{X}$ then follow from the expansions (F.10) of the $X$. Finally, we note that the relationship (E.6) and the explicit values (4.9) of the $x_{i}$ can be used to rewrite (F.25) as

$$
\left[\frac{d \Gamma}{d x_{e} d y_{e}}\right]_{x y y \bar{x}}^{(\Delta t<a)}=-\frac{N_{\mathrm{f}} \alpha_{\mathrm{EM}}^{2} M_{\mathrm{i}}^{2}}{8 \pi^{4} E^{2}} \frac{P_{e \rightarrow e}\left(x_{e}\right) P_{\gamma \rightarrow e}\left(\frac{y_{e}}{1-x_{e}}\right)}{x_{e} y_{e}\left(1-x_{e}-y_{e}\right)\left(1-x_{e}\right)^{3}} \int_{0}^{a} \frac{d(\Delta t)}{(\Delta t)^{2}} Z_{\mathrm{yy}} I_{1}^{\mathrm{new}}+O(a) .
$$

The overall factors of $P_{e \rightarrow e} P_{\gamma \rightarrow e}$ are the source of the similar overall factors in (4.25), except that we will need to generalize the derivation to $d=2-\epsilon$ transverse dimensions.

\section{F.3 Needed generalizations to $d=2-\epsilon$}

In the case of QCD $g \rightarrow g g g$, the dimensional regularization of diagrams was carried out in ACI3 [9]. We can adapt intermediate results from that paper if we (i) first look at its sequential diagram result for $x y \bar{x} \bar{y}$ [ACI3 eq. (5.10)], (ii) convert to QED by making the same modifications as in section F.1 of this paper, and then (iii) adapt the results to the virtual $x y y \bar{x}$ diagram as in (4.8). But, for the reasons described in section 4.3.3, we also need to backtrack in the derivation of ACI3 and never expand the Bessel functions $K_{d / 4}\left(\frac{1}{2}|M| \Omega B^{2}\right)$ [ACI3 eq. (4.15)]. The result is the following generalization of the divergent 
$Z_{\mathrm{yy}} I_{1}$ term of (F.1) to $d$ dimensions:

$$
\begin{aligned}
{\left[\frac{d \Gamma}{d x_{e} d y_{e}}\right]_{x y y \bar{x}}^{(\Delta t<a)}=} & -\left(\frac{\mu^{2}}{E}\right)^{\epsilon} \frac{N_{\mathrm{f}} \alpha_{\mathrm{EM}}^{2} M_{\mathrm{i}}^{2}}{2^{d+2} \pi^{2 d+1} i^{d} E^{2}} \Gamma^{2}\left(\frac{1}{2}+\frac{d}{4}\right)\left(-\hat{x}_{1} \hat{x}_{2} \hat{x}_{3} \hat{x}_{4}\right)^{d / 2}(d \bar{\alpha}+\bar{\beta}+\bar{\gamma}) \\
& \times \int_{0}^{a} \frac{d(\Delta t)}{(\Delta t)^{d}} \int_{\boldsymbol{B}^{\mathrm{y}^{\prime}}, \boldsymbol{B}^{\mathrm{y}}} \boldsymbol{B}^{\mathrm{y} \prime} \cdot \boldsymbol{B}^{\mathrm{y}}\left(\frac{\left|M_{\mathrm{i}}\right| \Omega_{\mathrm{i}}}{\left(B^{\mathrm{y}^{\prime}}\right)^{2}}\right)^{d / 4} K_{d / 4}\left(\frac{1}{2}\left|M_{\mathrm{i}}\right| \Omega_{\mathrm{i}}\left(B^{\mathrm{y} \prime}\right)^{2}\right) \\
& \times\left(\frac{\left|M_{\mathrm{i}}\right| \Omega_{\mathrm{i}}}{\left(B^{\mathrm{y}}\right)^{2}}\right)^{d / 4} K_{d / 4}\left(\frac{1}{2}\left|M_{\mathrm{i}}\right| \Omega_{\mathrm{i}}\left(B^{\mathrm{y}}\right)^{2}\right) \\
& \times Z_{\mathrm{yy}^{\prime}} \exp \left[-\frac{1}{2} \mathcal{X}_{\mathrm{y}}\left(B^{\mathrm{y}}\right)^{2}-\frac{1}{2} \mathcal{X}_{\mathrm{y}^{\prime}}\left(B^{\mathrm{y} \prime}\right)^{2}+\mathcal{X}_{\mathrm{yy}^{\prime}} \boldsymbol{B}^{\mathrm{y}} \cdot \boldsymbol{B}^{\mathrm{y} \prime}\right]+O(a), \quad
\end{aligned}
$$

where the $M_{\mathrm{i}}, \Omega_{\mathrm{i}}$ and $(\mathcal{X}, Y, Z)$ are defined exactly the same as for $d=2$.

To make contact with the derivation in ACI3, compare (F.30) above to the $Z$ term in ACI3 (4.14) for the QCD $x y \bar{y} \bar{x}$ diagram. The structure is the same. In addition to a factor of 2 related to converting the QCD group factors for $x y \bar{y} \bar{x}$ to QED, and the different way $(\bar{\alpha}, \bar{\beta}, \bar{\gamma})$ are contracted here to make $d \bar{\alpha}+\bar{\beta}+\bar{\gamma}$ for the $Z$ term of $x y y \bar{x}$, there is one minor change in the overall pre-factor concerning the powers of $\mu$ and $E$ in (F.30) above. $\mu$ is the renormalization scale, which we introduce by writing the $d$-dimensional coupling constant $g_{d}$, which has dimensions of $(\text { mass })^{\epsilon / 2}$, as

$$
g_{d}=\mu^{\epsilon / 2} g
$$

where $g$ is the dimensionless coupling constant for $\epsilon=0$. As a result, the dimensionless $\alpha_{\mathrm{EM}}^{2}$ in (F.30) is associated with a factor of $\mu^{2 \epsilon}$, as written explicitly in the pre-factor. The overall power of $E$ then follows from dimensional analysis if one takes the convention, as in ACI3 [9], that the $d$-dimensional generalizations of $(\bar{\alpha}, \bar{\beta}, \bar{\gamma})$ are defined to be dimensionless. ${ }^{44}$

We may now use the $d$-dimensional generalization $[9]^{45}$

$$
\bar{\alpha}+\frac{1}{d} \bar{\beta}+\frac{1}{d} \bar{\gamma}=\frac{P_{e \rightarrow e}^{(d)}\left(x_{e}\right)}{x_{e}^{2}\left(1-x_{e}\right)^{2}} \frac{P_{\gamma \rightarrow e}^{(d)}\left(\frac{y_{e}}{1-x_{e}}\right)}{\left(1-x_{e}\right) y_{e}^{2}\left(1-x_{e}-y_{e}\right)^{2}}
$$

\footnotetext{
${ }^{44}$ To wit, there was a mistake in the prefactors in ACI3 [9], which are off by an overall factor of $(\mu / E)^{2 \epsilon}$. This mistake did not matter there because the $1 / \epsilon$ poles all cancel when one adds up all of the real-double splitting diagrams (because there should be no UV divergence in the total result if there are no loops in the amplitude or in the conjugate amplitude). In that case, the $\epsilon$ dependence of an overall factor common to all diagrams will not matter when we set $\epsilon=0$ at the end of the day. In this paper, however, the $1 / \epsilon$ poles do not cancel when we sum the virtual diagrams of figure 8 - they can't, because we know we need to get coupling renormalization at this order. ACI3's error in the overall factor comes in the paragraph of ACI3 appendix A concerning ACI3 eq. (4.3), which forgets that the $d$-dimensional coupling is dimensionful.

${ }^{45}$ The argument for this relation is the same as that for ACI3 (5.17) [9]. However, fixing the overall normalization error described in footnote 44 of the current paper modifies the $(1-x)^{d-1}$ in the second denominator of that equation to $1-x$. Like the overall normalization, this correction does not affect any of ACI3's final results because of the cancellation of divergence there, but it is important for our current work.
} 
of (E.6) to rewrite (F.30) as

$$
\left[\frac{d \Gamma}{d x_{e} d y_{e}}\right]_{x y y \bar{x}}^{(\Delta t<a)}=\left(\frac{\mu^{2}}{E}\right)^{\epsilon} \frac{d N_{\mathrm{f}} \alpha_{\mathrm{EM}}^{2}}{2^{d+2} \pi^{2 d} i^{d}} \Gamma^{2}\left(\frac{1}{2}+\frac{d}{4}\right) \frac{P_{e \rightarrow e}^{(d)}\left(x_{e}\right) P_{\gamma \rightarrow e}^{(d)}\left(\mathfrak{y}_{e}\right)}{\left(1-x_{e}\right)\left[x_{e} y_{e}\left(1-x_{e}-y_{e}\right)\right]^{\epsilon / 2}} \mathbb{I}+O(a),
$$

where here $\mathbb{I}$ is defined by (4.30) with $(M, \Omega)$ set to $\left(M_{\mathrm{i}}, \Omega_{\mathrm{i}}\right)$. The explicit formulas (4.9) and (4.22) for $\hat{x}_{i}$ and $M_{\mathrm{i}}$ have been used above, as well as the small- $\Delta t$ expansion (F.6d) of $Z_{\mathrm{yy}^{\prime}} \cdot \mathfrak{y}_{e} \equiv y_{e} /\left(1-x_{e}\right)$ as in (2.16) is the longitudinal momentum fraction of the virtual pair's electron relative to the its immediate parent, the photon. When combined with the definition of $\mathbb{I}$, (F.33) supplies the proportionality constant that we did not show in the corresponding version (4.25) in the main text.

We will see later that we do not need the $d$-dimensional version of the DGLAP splitting function $P_{e \rightarrow e}^{(d)}\left(x_{e}\right)$ in (4.25) because it is common to $x y y \bar{x}$ and the leading-order process $x \bar{x}$. But we will need the $d$-dimensional version of the other DGLAP splitting function $P_{\gamma \rightarrow e}^{(d)}\left(\mathfrak{y}_{e}\right)$, which is ${ }^{46}$

$$
P_{\gamma \rightarrow e}^{(d)}(z)=z^{2}+(1-z)^{2}-\frac{2 \epsilon}{2-\epsilon} z(1-z),
$$

which reproduces the usual result in the case $\epsilon=0$.

\section{F.4 The subtraction $\mathcal{D}_{2}(\Delta t)$}

We now turn to the subtraction $\mathcal{D}_{2}(\Delta t)$ introduced in (4.16) and (4.17),

$$
\lim _{a \rightarrow 0}\left[\int_{0}^{a} d(\Delta t) F_{d}(\Delta t)+\int_{a}^{\infty} d(\Delta t) \mathcal{D}_{2}(\Delta t)\right]+\int_{0}^{\infty} d(\Delta t)\left[F_{2}(\Delta t)-\mathcal{D}_{2}(\Delta t)\right]+O(\epsilon),
$$

which will allow us to (i) turn the $d=2$ expression (F.1) for $x y y \bar{x}$ into a convergent integral that can be done numerically, corresponding to the $\int d(\Delta t)\left[F_{2}(\Delta t)-\mathcal{D}_{2}(\Delta t)\right]$ term above, and (ii) cancel the $a$ dependence of the $\Delta t<a$ contribution (F.33), as in the first two terms above. We will choose $\mathcal{D}_{2}(\Delta t)$ proportional to (4.18). To get the ultimate proportionality constant, see (F.25)-(F.27), but first we will define a $\mathcal{D}_{2}^{(\mathbb{I})}(\Delta t)$ by choosing a proportionality constant corresponding to the $\mathbb{I}_{\text {unregulated }}$ of (F.27):

$$
\mathcal{D}_{2}^{(\mathbb{I})}(\Delta t)=2 \pi^{2}\left[\frac{\ln \left(2 i \bar{\Omega}_{\mathrm{i}} \Delta t\right)}{(\Delta t)^{2}}-i \bar{\Omega}_{\mathrm{i}}^{3} \Delta t \csc ^{2}\left(\bar{\Omega}_{\mathrm{i}} \Delta t\right)\right] .
$$

This is equivalent to (A.44), given the definition (4.32) of $\bar{\Omega}_{\mathrm{i}}$.

\section{F.4.1 Combination with $\mathbb{I}$}

One of the integrals we need in (F.35) is then

$$
\int_{a}^{\infty} d(\Delta t) \mathcal{D}_{2}^{(\mathbb{I})}(\Delta t)=2 \pi^{2}\left(\frac{\ln \left(2 i \bar{\Omega}_{\mathrm{i}} a\right)+1}{a}+i \bar{\Omega}_{\mathrm{i}}\left[\ln \left(2 i \bar{\Omega}_{\mathrm{i}} a\right)-1\right]\right)+O(a) .
$$

\footnotetext{
${ }^{46}$ See, for example, eq. (16) of ref. [36], which one may verify independently. Our $\epsilon$ is their $2 \epsilon$, and their $T_{R}$ is 1 in the QED case we consider here. The result implicitly depends on the convention [37] that the trace of the Dirac identity matrix is simply defined as $\operatorname{tr}\left(\mathbb{1}_{\text {Dirac }}\right) \equiv 4$ in $d$ dimensions, which is part of Conventional Dimensional Regularization for fermions.
} 
Combining this with the result (4.31) for $\mathbb{I}$, we see that the $a$ dependence cancels, leaving

$$
\mathbb{I}+\int_{a}^{\infty} d(\Delta t) \mathcal{D}_{2}(\Delta t)=2 \pi^{2}\left(i \bar{\Omega}_{\mathrm{i}}\right)^{d-1}\left[-\left(\frac{2}{\epsilon}-\gamma_{\mathrm{E}}+\ln (4 \pi)\right)+4 \ln 2+3 \ln \pi-1\right]+O(a)+O(\epsilon) .
$$

Now multiply this by the prefactors shown in (F.33) to convert $\mathbb{I}$ into the $\Delta t<a$ result for $x y y \bar{x}$ to get

$$
\begin{aligned}
\lim _{a \rightarrow 0}\{ & \left.\left\{\frac{d \Gamma}{d x_{e} d y_{e}}\right]_{x y y \bar{x}}^{(\Delta t<a)}+\left[\frac{d \Gamma}{d x_{e} d y_{e}}\right]_{x y y \bar{x}}^{\left(\mathcal{D}_{2}\right)}\right\} \\
= & -\left(\frac{\mu^{2}}{E}\right)^{\epsilon} \frac{d N_{\mathrm{f}} \alpha_{\mathrm{EM}}^{2}}{2^{d+2} \pi^{2 d}} \Gamma^{2}\left(\frac{1}{2}+\frac{d}{4}\right) \frac{P_{e \rightarrow e}^{(d)}\left(x_{e}\right) P_{\gamma \rightarrow e}^{(d)}\left(\mathfrak{y}_{e}\right)}{\left(1-x_{e}\right)\left[x_{e} y_{e}\left(1-x_{e}-y_{e}\right)\right]^{\epsilon / 2}} \\
& \times 2 \pi^{2} i \bar{\Omega}_{\mathrm{i}}^{d-1}\left[-\left(\frac{2}{\epsilon}-\gamma_{\mathrm{E}}+\ln (4 \pi)\right)+4 \ln 2+3 \ln \pi-1\right]+O(\epsilon) .
\end{aligned}
$$

We'll leave it in this form for the moment.

\section{F.4.2 The subtracted piece}

Using the normalization of (F.26) for the relation between $\mathbb{I}_{\text {unregulated }}$ and $\int(\Delta t)^{-2} Z I_{1}$, the $\int d(\Delta t)\left[F_{2}(\Delta t)-\mathcal{D}_{2}(\Delta t)\right]$ term of (F.35) then corresponds to modifying (F.1) and (F.2) to

$$
\begin{aligned}
{\left[\frac{d \Gamma}{d x_{e} d y_{e}}\right]_{x y y \bar{x}}^{(\text {subtracted })}=- } & \frac{N_{\mathrm{f}} \alpha_{\mathrm{EM}}^{2} M_{\mathrm{i}}^{2}}{16 \pi^{4} E^{2}}\left(-\hat{x}_{1} \hat{x}_{2} \hat{x}_{3} \hat{x}_{4}\right) \int_{0}^{\infty} d(\Delta t)[ \\
& \frac{\Omega_{+} \csc \left(\Omega_{+} \Delta t\right)}{\Delta t}\left\{\left(\bar{\beta} Y_{\mathrm{y}}^{2}+\bar{\gamma} \bar{Y}_{\mathrm{yy}^{\prime}} Y_{\mathrm{yy}^{\prime}}\right) I_{0}^{\text {new }}+(2 \bar{\alpha}+\bar{\beta}+\bar{\gamma}) Z_{\mathrm{yy}} I_{1}^{\text {new }}\right. \\
& +\left[(\bar{\alpha}+\bar{\gamma}) Y_{\mathrm{y}}^{2}+(\bar{\alpha}+\bar{\beta}) \bar{Y}_{\mathrm{yy}^{\prime}} Y_{\mathrm{yy}^{\prime}}\right] I_{2}^{\text {new }} \\
& \left.-(\bar{\alpha}+\bar{\beta}+\bar{\gamma})\left(\bar{Y}_{\mathrm{yy}} Y_{\mathrm{y}} I_{3}^{\text {new }}+Y_{\mathrm{y}} Y_{\mathrm{yy}} I_{4}^{\text {new }}\right)\right\} \\
& \left.-(2 \bar{\alpha}+\bar{\beta}+\bar{\gamma}) \frac{\hat{x}_{2} \hat{x}_{3}}{\hat{x}_{1} \hat{x}_{2}} \mathcal{D}_{2}^{(\mathbb{I})}\right]
\end{aligned}
$$

As designed, this is an integral that can be done numerically.

\section{F.5 Integration over $y_{e}$}

For the virtual diagram, we need to integrate over $y_{e}$ as in (4.7). For the subtracted piece (F.40), this is another integral we will do numerically:

$$
\left[\frac{d I}{d x_{e}}\right]_{x y y \bar{x}}^{(\text {subtracted })}=\int_{0}^{1-x_{e}} d y_{e}\left[\frac{d I}{d x_{e} d y_{e}}\right]_{x y y \bar{x}}^{(\text {subtracted })}
$$


For the other piece (F.39), we will do the $y_{e}$ integral analytically. We start by changing integration variable from $y_{e}$ to $\mathfrak{y}_{e} \equiv y_{e} /\left(1-x_{e}\right)$ :

$$
\begin{aligned}
\lim _{a \rightarrow 0 "}\left\{\left[\frac{d \Gamma}{d x_{e}}\right]_{x y y \bar{x}}^{(\Delta t<a)}+\left[\frac{d \Gamma}{d x_{e}}\right]_{x y y \bar{x}}^{\left(\mathcal{D}_{2}\right)}\right\} \\
=-\left(\frac{\mu^{2}}{E}\right)^{\epsilon} \frac{d N_{\mathrm{f}} \alpha_{\mathrm{EM}}^{2}}{2^{d+2} \pi^{2 d}} \Gamma^{2}\left(\frac{1}{2}+\frac{d}{4}\right) \frac{P_{e \rightarrow e}^{(d)}\left(x_{e}\right)}{x_{e}^{\epsilon / 2}\left(1-x_{e}\right)^{\epsilon}} \int_{0}^{1} d \mathfrak{y}_{e} \frac{P_{\gamma \rightarrow e}^{(d)}\left(\mathfrak{y}_{e}\right)}{\left[\mathfrak{y}_{e}\left(1-\mathfrak{y}_{e}\right)\right]^{\epsilon / 2}} \\
\quad \times 2 \pi^{2} i \bar{\Omega}_{\mathrm{i}}^{d-1}\left[-\left(\frac{2}{\epsilon}-\gamma_{\mathrm{E}}+\ln (4 \pi)\right)+4 \ln 2+3 \ln \pi-1\right]+O(\epsilon) .
\end{aligned}
$$

$\Omega_{\mathrm{i}}$ does not depend on $y_{e}$, and the $\mathfrak{y}_{e}$ integral can be done using the formula (F.34) for $P_{\gamma \rightarrow e}^{(d)}$, giving

$$
\int_{0}^{1} d \mathfrak{y}_{e} \frac{P_{\gamma \rightarrow e}^{(d)}\left(\mathfrak{y}_{e}\right)}{\left[\mathfrak{y}_{e}\left(1-\mathfrak{y}_{e}\right)\right]^{\epsilon / 2}}=\frac{4 \Gamma\left(\frac{4-\epsilon}{2}\right)}{\Gamma(4-\epsilon)}=\frac{2}{3}+\frac{5}{9} \epsilon+O\left(\epsilon^{2}\right) .
$$

\section{F.6 Renormalization}

To carry out renormalization as in (4.38), we need the leading-order result $\left[d \Gamma / d x_{e}\right]_{x \bar{x}}$ in $d$ transverse dimensions. That can be taken from the similar result in ACI3 [9] for $g \rightarrow g g$ except that $C_{\mathrm{A}} \alpha_{\mathrm{S}} P_{g \rightarrow g g}(x)$ in QCD is replaced by $\alpha_{\mathrm{EM}} P_{e \rightarrow e}\left(x_{e}\right)$ here: ${ }^{47}$

$$
\left[\frac{d \Gamma}{d x_{e}}\right]_{x \bar{x}}=-\frac{\mu^{\epsilon} \alpha_{\mathrm{EM}} d}{8 \pi} P_{e \rightarrow e}^{(d)}\left(x_{e}\right) \mathrm{B}\left(\frac{1}{2}+\frac{d}{4},-\frac{d}{4}\right)\left(\frac{2 \pi}{M_{\mathrm{i}} \bar{\Omega}_{\mathrm{i}}}\right)^{\epsilon / 2} i \bar{\Omega}_{\mathrm{i}},
$$

where $\mathrm{B}(x, y) \equiv \Gamma(x) \Gamma(y) / \Gamma(x+y)$ is the Euler Beta function and the factor of $\mu^{\epsilon}$ associated with $\alpha_{\mathrm{EM}}$ comes from (F.31).

Using (4.33) and (F.42)-(F.44), and expanding as necessary in $\epsilon$, we can rewrite

$$
\left[\frac{d \Gamma}{d x_{e}}\right]_{x y y \bar{x}}=-\frac{N_{\mathrm{f}} \alpha_{\mathrm{EM}}}{3 \pi}\left[\frac{d \Gamma}{d x_{e}}\right]_{x \bar{x}}\left(\frac{2}{\epsilon}+\ln \left(\frac{\pi \mu^{2}}{\left(1-x_{e}\right) E \bar{\Omega}_{\mathrm{i}}}\right)+\frac{5}{3}\right)+\left[\frac{d \Gamma}{d x_{e}}\right]_{x y y \bar{x}}^{\text {(subtracted) }} .
$$

Renormalizing using (4.38) then leaves us with

$$
\begin{aligned}
{\left[\frac{d \Gamma}{d x_{e}}\right]_{x y y \bar{x}}^{(\mathrm{ren})}=} & -\frac{N_{\mathrm{f}} \alpha_{\mathrm{EM}}}{3 \pi}\left[\frac{d \Gamma}{d x_{e}}\right]_{x \bar{x}}\left(\ln \left(\frac{\mu^{2}}{\left(1-x_{e}\right) E \bar{\Omega}_{\mathrm{i}}}\right)+\gamma_{\mathrm{E}}-2 \ln 2+\frac{5}{3}\right) \\
& +\int_{0}^{1-x_{e}} d y_{e}\left[\frac{d \Gamma}{d x_{e} d y_{e}}\right]_{x y y \bar{x}}^{(\text {subtracted })}
\end{aligned}
$$

in which $\left[d \Gamma / d x_{e} d y_{e}\right]_{x y y \bar{x}}^{\text {(subtracted) }}$ is given by (F.40). Eq. (F.46) is the result (4.39) quoted in the main text, but generalized here to handle either sign of $M_{\mathrm{i}}$ and so handle front-end transformations such as in (4.40). Since the renormalized result (4.39) is finite, we may use the $d=2$ version (A.42) of (F.44) there, which is

$$
\left[\frac{d \Gamma}{d x_{e}}\right]_{x \bar{x}}=\frac{\alpha_{\mathrm{EM}}}{2 \pi} P_{e \rightarrow e}\left(x_{e}\right) i \bar{\Omega}_{\mathrm{i}} \quad \text { for } d=2
$$

\footnotetext{
${ }^{47}$ Specifically, ACI3 eqs. (3.1), (3.2) and (3.7) [9] give $2 \operatorname{Re}[d \Gamma / d x]_{x \bar{x}}$. We've used the more general $\bar{\Omega}_{\mathrm{i}}$ (4.32) instead of $\Omega_{\mathrm{i}}$ to fit how we've written results for $x y y \bar{x}$, which we want to have the option of frontand back-end transforming via transforming via (4.40).
} 
So, as previously promised, we never need the $d$-dimensional version of the structure function factor $P_{e \rightarrow e}^{(d)}\left(x_{e}\right)$ that was common to the leading-order $x \bar{x}$ and the virtual correction $x y y \bar{x}$.

\section{F.7 $\hat{q}$ and dimensional regularization}

Throughout this paper, we have used $\hat{q}$ as an independent parameter describing interactions with the medium, which could either be calculated theoretically (in certain limiting cases) or used as a phenomenological parameter. One might worry whether or not the use of dimensional regularization requires knowing the $O(\epsilon)$ corrections to the $3+1$ dimensional value of $\hat{q}$, given that various of our formulas along the way have involved $1 / \epsilon$ divergences multiplying expressions that depend on $\hat{q}$ through complex frequencies $\Omega$. Fortunately, this is not an issue. Imagine that we used the full $d$-dimensional value of $\hat{q}$ (whatever it is) everywhere in our intermediate calculations. Since our final, renormalized results are finite expressions where the $\epsilon \rightarrow 0$ limit is taken, the $\hat{q}$ in those expressions can then be replaced in the last step by $3+1$ dimensional $\hat{q}$, just as we $\operatorname{did}$ for $P_{e \rightarrow e}\left(x_{e}\right)$ above. $^{48}$

\section{G The integral $\mathbb{I}$}

In this appendix, we derive the result (4.31) for the $\mathbb{I}$ integral defined by (4.30).

\section{G.1 Perturbative treatment of $O\left(M \Omega^{2} \Delta t B^{2}\right)$ corrections to the exponent}

The definition (4.30) of $\mathbb{I}$ is

$$
\begin{gathered}
\mathbb{I} \equiv \frac{i M(|M| \Omega)^{d / 2}}{\pi} \int_{0}^{a} \frac{d(\Delta t)}{(\Delta t)^{d+1}} \int_{\boldsymbol{B}, \boldsymbol{B}^{\prime}} \frac{\boldsymbol{B} \cdot \boldsymbol{B}^{\prime}}{\left(B^{2}\right)^{d / 4}\left(B^{\prime 2}\right)^{d / 4}} K_{d / 4}\left(\frac{1}{2}|M| \Omega B^{2}\right) K_{d / 4}\left(\frac{1}{2}|M| \Omega B^{\prime 2}\right) \\
\times \exp \left[-\frac{1}{2} \mathcal{X}_{\mathrm{y}} B^{2}-\frac{1}{2} \mathcal{X}_{\mathrm{y}^{\prime}}{B^{\prime 2}}^{2} \mathcal{X}_{\mathrm{yy}} \boldsymbol{B} \cdot \boldsymbol{B}^{\prime}\right],
\end{gathered}
$$

where the $\mathcal{X}$ have the small- $\Delta t$ expansions (4.26)

$$
\begin{aligned}
\mathcal{X}_{\mathrm{y}}=\mathcal{X}_{\mathrm{y}^{\prime}} & =-\frac{i M}{\Delta t}+\frac{i M \Omega^{2} \Delta t}{3}+\cdots, \\
\mathcal{X}_{\mathrm{yy}^{\prime}} & =-\frac{i M}{\Delta t}-\frac{i M \Omega^{2} \Delta t}{6}+\cdots .
\end{aligned}
$$

Plugging these expansions into the exponential of (G.1) gives

$$
\exp \left[-\frac{1}{2} \mathcal{X}_{\mathrm{y}} B^{2}-\frac{1}{2} \mathcal{X}_{\mathrm{y}^{\prime}}{B^{\prime 2}}^{2}+\mathcal{X}_{\mathrm{yy}^{\prime}} \boldsymbol{B} \cdot \boldsymbol{B}^{\prime}\right]=\exp \left[\frac{i M}{2 \Delta t}\left(\boldsymbol{B}-\boldsymbol{B}^{\prime}\right)^{2}\right] \exp \left[O\left(M \Omega^{2} \Delta t\left\{B^{2},{B^{\prime}}^{2}\right\}\right)\right],
$$

where the last exponential factor represents the corrections from the second terms in the expansions (G.2) and $O(\cdots)$ means "of order." Because of the bound (4.29) on the sizes of both $B$ and $B^{\prime}$ that contribute to $\mathbb{I}$, the exponent of this second exponential is small:

$$
\left|M \Omega^{2} \Delta t B^{2}\right| \lesssim|\Omega \Delta t| \leq|\Omega a| \ll 1
$$

\footnotetext{
${ }^{48}$ We are assuming here that $\hat{q}$ has been defined in a physically relevant way so that it itself is not infinite in $3+1$ dimensions. See appendix C.
} 
We will need this correction, but we can treat it as perturbative, rewriting (G.3) as

$$
\exp \left[-\frac{1}{2} \mathcal{X}_{\mathrm{y}} B^{2}-\frac{1}{2} \mathcal{X}_{\mathrm{y}^{\prime}}{B^{\prime 2}}^{2}+X_{\mathrm{yy}} \boldsymbol{B} \cdot \boldsymbol{B}^{\prime}\right]=\exp \left[\frac{i M}{2 \Delta t}\left(\boldsymbol{B}-\boldsymbol{B}^{\prime}\right)^{2}\right]\left[1+O\left(M \Omega^{2} \Delta t\left\{B^{2},{B^{\prime}}^{2}\right\}\right)\right] .
$$

Formally, it will be convenient to implement this expansion by introducing a redundant parameter $\xi=1$ into (G.2),

$$
\begin{aligned}
\mathcal{X}_{\mathrm{y}}=\mathcal{X}_{\mathrm{y}^{\prime}} & =-\frac{i M}{\Delta t}+\xi \frac{i M \Omega^{2} \Delta t}{3}+O\left(M \Omega^{4}(\Delta t)^{3}\right), \\
\mathcal{X}_{\mathrm{yy}^{\prime}} & =-\frac{i M}{\Delta t}-\xi \frac{i M \Omega^{2} \Delta t}{6}+O\left(M \Omega^{4}(\Delta t)^{3}\right),
\end{aligned}
$$

and then think of $\mathbb{I}$ as a function $\mathbb{I}(\xi)$ of $\xi$. The above discussion then translates to

$$
\mathbb{I}=[\mathbb{I}(\xi)]_{\xi=1}=\left[\mathbb{I}(0)+\xi \mathbb{I}^{\prime}(0)+\frac{\xi^{2}}{2 !} \mathbb{I}^{\prime \prime}(0)+\cdots\right]_{\xi=1}=\mathbb{I}(0)+\mathbb{I}^{\prime}(0)+O(a) .
$$

For the same reason that the unregulated $(\epsilon=0)$ discussion of appendix F.2 did not require the yet-higher order terms not explicitly shown in (G.6), we will not need them here either. They give vanishing contribution to $\mathbb{I}$ for $a \rightarrow 0$.

\section{G.2 Units}

In order to simplify the presentation of calculations, in this appendix we will start by assuming $M>0$ and evaluate $\mathbb{I}$ in units where

$$
M=1 \quad \text { and } \quad \Omega=1 .
$$

At the end, we will be able to put $M$ and the complex-valued $\Omega$ back into the answer using the scaling properties of the definition (G.1) of $\mathbb{I}$ and then analytic continuation of $\Omega$ back to complex values. Then we will generalize the result to also cover the case $M<0$.

\section{G.3 Representation of Bessel functions}

Integrating complicated things involving Bessel functions is hard, which motivates us to replace each Bessel function with the integral representation ${ }^{49}$

$$
z^{-\nu} K_{\nu}(z)=\frac{\pi^{1 / 2}}{2^{\nu} \Gamma\left(\frac{1}{2}+\nu\right)} \int_{1}^{\infty} d p\left(p^{2}-1\right)^{\nu-\frac{1}{2}} e^{-p z} \quad(z>0) .
$$

So (G.1) for $\mathbb{I}$ becomes

$$
\begin{aligned}
\mathbb{I}= & \frac{i 2^{-d}}{\Gamma^{2}\left(\frac{d+2}{4}\right)} \int_{1}^{\infty} d p d q\left(p^{2}-1\right)^{-\epsilon / 4}\left(q^{2}-1\right)^{-\epsilon / 4} \\
& \times \int_{0}^{a} \frac{d(\Delta t)}{(\Delta t)^{d+1}} \int_{\boldsymbol{B}, \boldsymbol{B}^{\prime}} \boldsymbol{B} \cdot \boldsymbol{B}^{\prime} \exp \left[-\frac{1}{2}\left(\mathcal{X}_{\mathrm{y}}+p\right) B^{2}-\frac{1}{2}\left(\mathcal{X}_{\mathrm{y}^{\prime}}+q\right) B^{\prime 2}+X_{\mathrm{yy}} \boldsymbol{B} \cdot \boldsymbol{B}^{\prime}\right] .
\end{aligned}
$$

\footnotetext{
${ }^{49}$ See, e.g., Gradshteyn \& Ryzhik (8.432.3) [38] for a more precise statement on range of validity.
} 


\section{G.4 Doing the $B$ integrals}

To do the $\boldsymbol{B}$ integrals above, it is convenient to take advantage of the form of the exponential to replace

$$
\boldsymbol{B} \cdot \boldsymbol{B}^{\prime} \exp [\cdots] \rightarrow \frac{\partial}{\partial \mathcal{X}_{\mathrm{yy}^{\prime}}} \exp [\cdots]
$$

The $\boldsymbol{B}$ integral of the exponential gives

$$
\begin{aligned}
& \int_{\boldsymbol{B}, \boldsymbol{B}^{\prime}} \exp \left[-\frac{1}{2}\left(\mathcal{X}_{\mathrm{y}}+p\right) B^{2}-\frac{1}{2}\left(\mathcal{X}_{\mathrm{y}^{\prime}}+q\right){B^{\prime}}^{2}+\mathcal{X}_{\mathrm{yy}} \boldsymbol{B} \cdot \boldsymbol{B}^{\prime}\right] \\
& =(2 \pi)^{d}\left[\operatorname{det}\left(\begin{array}{cc}
\mathcal{X}_{\mathrm{y}}+p & -\mathcal{X}_{\mathrm{yy}^{\prime}} \\
-\mathcal{X}_{\mathrm{yy}^{\prime}} & \mathcal{X}_{\mathrm{y}^{\prime}}+q
\end{array}\right)\right]^{-d / 2} \\
& =\frac{(2 \pi)^{d}}{\left[\left(\mathcal{X}_{\mathrm{y}} \mathcal{X}_{\mathrm{y}^{\prime}}-\mathcal{X}_{\mathrm{yy}^{\prime}}^{2}\right)+\mathcal{X}_{\mathrm{y}^{\prime}} p+\mathcal{X}_{\mathrm{y}} q+p q\right]^{d / 2}} \text {. }
\end{aligned}
$$

Now use (G.11), giving

$$
\begin{aligned}
\int_{\boldsymbol{B}, \boldsymbol{B}^{\prime}} \boldsymbol{B} \cdot \boldsymbol{B}^{\prime} \exp \left[-\frac{1}{2}\left(\mathcal{X}_{\mathrm{y}}+p\right) B^{2}-\right. & \left.\frac{1}{2}\left(\mathcal{X}_{\mathrm{y}^{\prime}}+q\right) B^{\prime 2}+\mathcal{X}_{\mathrm{yy}} \boldsymbol{B} \cdot \boldsymbol{B}^{\prime}\right] \\
= & \frac{d(2 \pi)^{d} \mathcal{X}_{\mathrm{yy}^{\prime}}}{\left[\left(\mathcal{X}_{\mathrm{y}^{\prime}} \mathcal{X}_{\mathrm{y}^{\prime}}-\mathcal{X}_{\mathrm{yy}^{\prime}}^{2}\right)+\mathcal{X}_{\mathrm{y}^{\prime}} p+\mathcal{X}_{\mathrm{y}} q+p q\right]^{\frac{d}{2}+1}} .
\end{aligned}
$$

Now specialize to our case by using (G.6) to get

$$
\begin{aligned}
\int_{\boldsymbol{B}, \boldsymbol{B}^{\prime}} \boldsymbol{B} \cdot \boldsymbol{B}^{\prime} \exp \left[-\frac{1}{2}\left(\mathcal{X}_{\mathrm{y}}+p\right) B^{2}-\right. & \left.\frac{1}{2}\left(\mathcal{X}_{\mathrm{y}^{\prime}}+q\right) B^{\prime 2}+\mathcal{X}_{\mathrm{yy}} \boldsymbol{B} \cdot \boldsymbol{B}^{\prime}\right] \\
= & \frac{-i d(2 \pi)^{d}}{\Delta t\left[-\frac{i(p+q)}{\Delta t}+p q+\xi\right]^{\frac{d}{2}+1}}\left[1+O\left((\Delta t)^{2}\right)\right] .
\end{aligned}
$$

As discussed earlier, the relative $O\left((\Delta t)^{2}\right)$ corrections can be dropped because they will lead to a convergent $\Delta t$ integral in $d=2$ that vanishes as $a \rightarrow 0$.

Using (G.14) in (G.10) gives

$$
\begin{aligned}
\mathbb{I}(\xi)= & \frac{d \pi^{d}}{\Gamma^{2}\left(\frac{d+2}{4}\right)} \int_{1}^{\infty} d p d q\left(p^{2}-1\right)^{-\epsilon / 4}\left(q^{2}-1\right)^{-\epsilon / 4} \\
& \times \int_{0}^{a} \frac{d(\Delta t)}{(\Delta t)^{(d+2) / 2}}[-i(p+q)+(p q+\xi) \Delta t]^{-(d+2) / 2}+O(a) .
\end{aligned}
$$

We find it convenient to change integration variables to

$$
u=\frac{1}{p}, \quad v=\frac{1}{q},
$$


to get $^{50}$

$$
\begin{aligned}
\mathbb{I}(\xi)= & \frac{d \pi^{d}}{\Gamma^{2}\left(\frac{d+2}{4}\right)} \int_{0}^{1} d u d v\left(1-u^{2}\right)^{-\epsilon / 4}\left(1-v^{2}\right)^{-\epsilon / 4} \\
& \times \int_{0}^{a} \frac{d(\Delta t)}{(\Delta t)^{(d+2) / 2}}[-i(u+v)+(1+\xi u v) \Delta t]^{-(d+2) / 2}+O(a) .
\end{aligned}
$$

\section{G.5 Doing the $\Delta t$ integral}

To perform the $\Delta t$ integrals, we use $\mathrm{e}^{51}$

$$
\int_{0}^{a} \frac{d(\Delta t)}{(\Delta t)^{r}(\beta+\alpha \Delta t)^{s}}=\frac{a^{1-r} \beta^{-s}}{(1-r)} F\left(1-r, s ; 2-r ;-\frac{\alpha}{\beta} a\right),
$$

where $F={ }_{2} F_{1}$ is the hypergeometric function. In our application (G.17),

$$
\begin{gathered}
\int_{0}^{a} \frac{d(\Delta t)}{(\Delta t)^{(d+2) / 2}}[-i(u+v)+(1+\xi u v) \Delta t]^{-(d+2) / 2} \\
\quad=-\frac{2}{d a^{d / 2} \beta^{(d+2) / 2}} F\left(-\frac{d}{2}, 1+\frac{d}{2} ; 1-\frac{d}{2} ;-\frac{\alpha}{\beta} a\right)
\end{gathered}
$$

with

$$
\alpha \equiv 1+\xi u v, \quad \beta \equiv-i(u+v)
$$

(not to be confused with any other use of the letters $\alpha$ or $\beta$ in this paper, but there are only so many letters in the alphabet). Use the hypergeometric transformation

$$
\begin{aligned}
F(a, b ; c ; z)= & \frac{\Gamma(c) \Gamma(b-a)}{\Gamma(b) \Gamma(c-a)}(-z)^{-a} F\left(a, 1-c+a ; 1-b+a ; \frac{1}{z}\right) \\
& +\frac{\Gamma(c) \Gamma(a-b)}{\Gamma(a) \Gamma(c-b)}(-z)^{-b} F\left(b, 1-c+b ; 1-a+b ; \frac{1}{z}\right),
\end{aligned}
$$

together with $F(a, 0 ; c ; z)=1$ [which follows from the series expansion that defines the hypergeometric function], to rewrite (G.19) as

$$
\begin{aligned}
& \int_{0}^{a} \frac{d(\Delta t)}{(\Delta t)^{(d+2) / 2}}[-i(u+v)+(1+\xi u v) \Delta t]^{-(d+2) / 2} \\
& \quad=-\frac{2}{d}\left[\frac{\Gamma\left(1-\frac{d}{2}\right) \Gamma(1+d)}{\Gamma\left(1+\frac{d}{2}\right)} \frac{\alpha^{d / 2}}{\beta^{1+d}}+\frac{\Gamma\left(1-\frac{d}{2}\right) \Gamma(-1-d)}{\Gamma\left(-\frac{d}{2}\right) \Gamma(-d)} \frac{F\left(1+\frac{d}{2}, 1+d ; 2+d ;-\frac{\beta}{\alpha a}\right)}{a^{1+d} \alpha^{1+\frac{d}{2}}}\right] \\
& \quad=-\frac{2 \Gamma\left(1-\frac{d}{2}\right) \Gamma(1+d)}{d \Gamma\left(1+\frac{d}{2}\right)} \frac{\alpha^{d / 2}}{\beta^{1+d}}-\frac{F\left(1+\frac{d}{2}, 1+d ; 2+d ;-\frac{\beta}{\alpha a}\right)}{(1+d) a^{1+d} \alpha^{1+\frac{d}{2}}} .
\end{aligned}
$$

The advantage of this rewriting is that the second term on the right-hand side of (G.22) is finite if we set $d=2$. That is, the $1 / \epsilon$ divergence arising from the $\Delta t$ integration is

\footnotetext{
${ }^{50}$ One check of (G.17) is to set $d=2$ and $\xi=1$, do the $(u, v)$ integrals, and verify that the result reproduces (F.27).

${ }^{51}$ The integral (G.18) can be derived by expanding the integrand in a Taylor series in $\alpha$ and integrating term by term.
} 
isolated in the first term. Moreover, we will see that the integration of the finite second term over $(u, v)$ is also finite, so we do not need to keep dimensional regularization in order to do those $(u, v)$ integrals: we can just set $d=2$ there and be done with it. All together, using (G.22) in the expression (G.17) for $\mathbb{I}$ gives

$$
\mathbb{I}(\xi)=\mathbb{I}_{\text {div }}(\xi)+\mathbb{I}_{\text {regular }}(\xi)+O(a)+O(\epsilon)
$$

with (dimensionally regularized) divergent piece

$$
\begin{aligned}
\mathbb{I}_{\text {div }}(\xi) & =-\frac{d \pi^{d}}{\Gamma^{2}\left(\frac{d+2}{4}\right)} \int_{0}^{1} d u d v\left(1-u^{2}\right)^{-\epsilon / 4}\left(1-v^{2}\right)^{-\epsilon / 4} \frac{2 \Gamma\left(1-\frac{d}{2}\right) \Gamma(1+d)}{d \Gamma\left(1+\frac{d}{2}\right)} \frac{\alpha^{d / 2}}{\beta^{1+d}} \\
& =-\frac{2 \pi^{d} \Gamma\left(1-\frac{d}{2}\right) \Gamma(1+d)}{\Gamma\left(1+\frac{d}{2}\right) \Gamma^{2}\left(\frac{d+2}{4}\right)} \int_{0}^{1} d u d v\left(1-u^{2}\right)^{-\epsilon / 4}\left(1-v^{2}\right)^{-\epsilon / 4} \frac{(1+\xi u v)^{d / 2}}{[-i(u+v)]^{1+d}}
\end{aligned}
$$

and regular, finite piece

$$
\mathbb{I}_{\text {regular }}(\xi)=-2 \pi^{2} \int_{0}^{1} d u d v \frac{F\left(2,3 ; 4 ;-\frac{\beta}{\alpha a}\right)}{3 a^{3} \alpha^{2}}=-\frac{2 \pi^{2}}{3 a^{3}} \int_{0}^{1} d u d v \frac{F\left(2,3 ; 4 ; \frac{i(u+v)}{(1+\xi u v) a}\right)}{(1+\xi u v)^{2}} .
$$

The hypergeometric function above is given in terms of elementary functions as

$$
F(2,3 ; 4 ; z)=\frac{6\left[\ln (1-z)+z+\frac{1}{2} z^{2}\right]}{z^{3}}+\frac{3}{1-z}
$$

and falls like $3 / z^{2}$ for large $z$.

\section{G.6 Evaluating $\mathbb{I}_{\text {regular }}$}

We have not investigated whether the $(u, v)$ integrals (G.25) for $\mathbb{I}_{\text {regular }}(\xi)$ can be performed by brute force, but the integrals simplify if we use the trick of expanding in $\xi$ as in (G.7):

$$
\mathbb{I}=\mathbb{I}(0)+\mathbb{I}^{\prime}(0)+O(a)
$$

One can use symbolic integration software to do the integrals corresponding to $\mathbb{I}_{\text {regular }}(0)$ and $\mathbb{I}_{\text {regular }}^{\prime}(0)$. Alternatively, one can rewrite $d u d v=\frac{1}{2} d(u+v) d(u-v)$ with appropriate limits of integration and do the relatively simple $u-v$ integration by hand, followed by the $u+v$ integration. By either method, the results, when expanded in $a$, are

$$
\begin{aligned}
& \mathbb{I}_{\text {regular }}(0)=-\frac{2 \pi^{2}}{a}[\ln (2 i a)+1]-i \pi^{2}[3 \ln (i a)+\ln 2-3]+O(a), \\
& \mathbb{I}_{\text {regular }}^{\prime}(0)=i \pi^{2}[\ln (i a)+3 \ln 2]+O(a),
\end{aligned}
$$

so that

$$
\mathbb{I}_{\text {regular }}(0)+\mathbb{I}_{\text {regular }}^{\prime}(0)=-\frac{2 \pi^{2}}{a}[\ln (2 i a)+1]-i \pi^{2}\left[2 \ln \left(\frac{i a}{2}\right)-3\right]+O(a) .
$$

The ignorability of higher-order terms in the $\xi$-expansion (G.27) of $\mathbb{I}$ in the $a \rightarrow 0$ limit will hold for the total $\mathbb{I}=\mathbb{I}_{\text {div }}+\mathbb{I}_{\text {regular }}$ but turns out not to hold separately for $\mathbb{I}_{\text {div }}$ 
and $\mathbb{I}_{\text {regular. A }}$. check we should make on our calculation is that the $O\left(\xi^{2}, \xi^{3}, \cdots\right)$ terms indeed cancel between $\mathbb{I}_{\text {div }}$ and $\mathbb{I}_{\text {regular }}$. For this purpose, it will be sufficient to check that $\mathbb{I}_{\text {div }}^{\prime \prime}(\xi)+\mathbb{I}_{\text {regular }}^{\prime \prime}(\xi)=O(a)$ for arbitrary $\xi \leq 1$. So, for later reference, we note here that differentiating the integrand of (G.25) twice with respect to $\xi$ gives

$$
\begin{aligned}
\mathbb{I}_{\text {regular }}^{\prime \prime}(\xi) & =4 \pi^{2} i \int_{0}^{1} d u d v \frac{(u v)^{2}}{(u+v+i(1+\xi u v) a)^{3}(1+\xi u v)} \\
& =4 \pi^{2} i \int_{0}^{1} d u d v \frac{(u v)^{2}}{(u+v)^{3}(1+\xi u v)}+O(a) .
\end{aligned}
$$

\section{G.7 Evaluating $\mathbb{I}_{\text {div }}$}

Rewrite (G.24) as

$$
\begin{aligned}
\mathbb{I}_{\text {div }}(\xi) & =-\frac{2 \pi^{d} \Gamma\left(1-\frac{d}{2}\right) \Gamma(1+d)}{\Gamma\left(1+\frac{d}{2}\right) \Gamma^{2}\left(\frac{d+2}{4}\right)} i^{d+1} \mathbb{A}(\xi) \\
& =4 \pi^{2} i\left(\frac{2}{\epsilon}-\gamma_{\mathrm{E}}-2 \ln (i \pi)-2+O(\epsilon)\right) \mathbb{A}(\xi),
\end{aligned}
$$

where

$$
\mathbb{A}(\xi) \equiv \int_{0}^{1} d u d v\left(1-u^{2}\right)^{-\epsilon / 4}\left(1-v^{2}\right)^{-\epsilon / 4} \frac{(1+\xi u v)^{d / 2}}{(u+v)^{1+d}} .
$$

As before, we take the $\xi$ expansion (G.27) of $\mathbb{I}$ and so here of $\mathbb{A}$. The integral

$$
\mathbb{A}(0)=\int_{0}^{1} \frac{d u d v}{(u+v)^{1+d}}\left(1-u^{2}\right)^{-\epsilon / 4}\left(1-v^{2}\right)^{-\epsilon / 4}
$$

does not converge for $d=2-\epsilon$ near 2 , and so we cannot simply expand the integrand in powers of $\epsilon$. Dimensional regularization tells us to imagine doing the integral in dimensions $d<1$ where it converges and then analytically continuing the result in $d$. But we can make that job easier if we first rewrite (G.34) as

$$
\mathbb{A}(0)=\int_{0}^{1} \frac{d u d v}{(u+v)^{1+d}}+\int_{0}^{1} \frac{d u d v}{(u+v)^{1+d}}\left[\left(1-u^{2}\right)^{-\epsilon / 4}\left(1-v^{2}\right)^{-\epsilon / 4}-1\right]
$$

The first integral is relatively easy, whereas the second integral converges for $d=2-\epsilon$ near 2 and so we may expand that integrand in powers of $\epsilon$. So, using $u \leftrightarrow v$ symmetry of the integrand,

$$
\begin{aligned}
\mathbb{A}(0) & =\int_{0}^{1} \frac{d u d v}{(u+v)^{1+d}}-\frac{\epsilon}{2} \int_{0}^{1} \frac{d u d v}{(u+v)^{3}} \ln \left(1-u^{2}\right)+O\left(\epsilon^{2}\right) \\
& =\frac{2\left(1-2^{-d}\right)}{d(1-d)}-\frac{\epsilon}{2}\left(-\frac{1}{4}-\frac{\ln 2}{2}\right)+O\left(\epsilon^{2}\right) \\
& =-\frac{3}{4}\left[1+\left(\frac{4}{3}-\frac{2}{3} \ln 2\right) \epsilon+O\left(\epsilon^{2}\right)\right]
\end{aligned}
$$

The next term in the $\xi$ expansion is

$$
\mathbb{A}^{\prime}(0)=\frac{d}{2} \int_{0}^{1} d u d v \frac{u v}{(u+v)^{1+d}}\left(1-u^{2}\right)^{-\epsilon / 4}\left(1-v^{2}\right)^{-\epsilon / 4}
$$


This integral is convergent for $d$ near 2, and so we may expand the integrand in powers of $\epsilon$. Again using $u \leftrightarrow v$ symmetry,

$$
\begin{aligned}
\mathbb{A}^{\prime}(0)= & \frac{d}{2}\left[\int_{0}^{1} d u d v \frac{u v}{(u+v)^{3}}-\frac{\epsilon}{2} \int_{0}^{1} d u d v \frac{u v}{(u+v)^{3}} \ln \left(1-u^{2}\right)\right. \\
& \left.+\epsilon \int_{0}^{1} d u d v \frac{u v}{(u+v)^{3}} \ln (u+v)\right] \\
= & \frac{d}{2}\left[\frac{1}{4}-\frac{\epsilon}{2}\left(\frac{1}{4}-\frac{1}{2} \ln 2\right)+\epsilon\left(\frac{3}{8}-\frac{3}{4} \ln 2\right)\right] \\
= & \frac{1}{4}\left[1+\left(\frac{1}{2}-2 \ln 2\right) \epsilon+O\left(\epsilon^{2}\right)\right] .
\end{aligned}
$$

Combining this with (G.36) gives

$$
\mathbb{A}(0)+\mathbb{A}^{\prime}(0)=-\frac{1}{2}\left[1+\frac{7}{4} \epsilon+O\left(\epsilon^{2}\right)\right]
$$

and thence, from (G.32),

$$
\mathbb{I}_{\mathrm{div}}(0)+\mathbb{I}_{\mathrm{div}}^{\prime}(0)=2 \pi^{2} i\left[-\frac{2}{\epsilon}+\gamma_{\mathrm{E}}+2 \ln (i \pi)-\frac{3}{2}+O(\epsilon)\right] .
$$

Let's also pause to check the cancellation of higher-order terms $O\left(\xi^{2}, \xi^{3}, \cdots\right)$ in the $\xi$ expansion. Differentiating (G.24) twice with respect to $\xi$ gives

$$
\mathbb{I}_{\text {div }}^{\prime \prime}(\xi)=-\frac{2 \pi^{d} \Gamma\left(1-\frac{d}{2}\right) \Gamma(1+d)}{\Gamma\left(1+\frac{d}{2}\right) \Gamma^{2}\left(\frac{d+2}{4}\right)} \int_{0}^{1} d u d v\left(1-u^{2}\right)^{-\epsilon / 4}\left(1-v^{2}\right)^{-\epsilon / 4} \frac{\frac{d}{2}\left(\frac{d}{2}-1\right)(1+\xi u v)^{\frac{d}{2}-2}(u v)^{2}}{[-i(u+v)]^{1+d}} .
$$

The integral is convergent for $d$ near 2, and expanding in $\epsilon$ gives

$$
\mathbb{I}_{\text {div }}^{\prime \prime}(\xi)=-4 \pi^{2} i \int_{0}^{1} d u d v \frac{(u v)^{2}}{(u+v)^{3}(1+\xi u v)}+O(\epsilon) .
$$

As promised, this indeed cancels the corresponding behavior (G.31) up to $O(a)$ corrections, confirming the expansion $\mathbb{I}=\mathbb{I}(0)+\mathbb{I}^{\prime}(0)+O(a)$ for the total $\mathbb{I}=\mathbb{I}_{\text {div }}+\mathbb{I}_{\text {regular }}$.

\section{G.8 Final result for $\mathbb{I}$ for $M>0$}

Combining (G.7), (G.23), (G.30), and (G.40), we get the result for the integral $\mathbb{I}$ defined by $(4.30)$ :

$$
\mathbb{I}=2 \pi^{2} i\left[-\left(\frac{2}{\epsilon}-\gamma_{\mathrm{E}}+\ln (4 \pi)\right)-\frac{\ln (2 i a)+1}{i a}-\ln a+3 \ln (2 \pi)+\frac{i \pi}{2}\right]
$$

in units where $M=1$ and $\Omega=1$. We have isolated the combination $\frac{2}{\epsilon}-\gamma_{\mathrm{E}}+\ln (4 \pi)$ above because that is the combination that appears in $\overline{\mathrm{MS}}$ renormalization (4.35).

To restore $M$ and $\Omega$, note that we could scale out all the $M$ and $\Omega$ from the original integral (G.1) combined with (G.2) by rescaling integration variables as

$$
\boldsymbol{B} \rightarrow \frac{\boldsymbol{B}}{(M \Omega)^{1 / 2}}, \quad \Delta t \rightarrow \frac{\Delta t}{\Omega} .
$$


This rescaling brings (G.1) into a dimensionless form times (i) the $M(M \Omega)^{d / 2}$ already explicit in (G.1), (ii) a factor of $\Omega^{d}$ from the $d(\Delta t) /(\Delta t)^{d+1}$, and (iii) a factor of $(M \Omega)^{-(d+2) / 2}$ from the $d^{d} B d^{d} B^{\prime} \boldsymbol{B} \cdot \boldsymbol{B}^{\prime} /\left(B^{2}\right)^{d / 4}\left(B^{\prime 2}\right)^{d / 4}$. These combine into an overall factor of $\Omega^{d-1}$. We can therefore go backward and restore the original units to (G.43) by (a) replacing $a$ (which is a cut-off on $\Delta t$ ) by $\Omega a$ and (b) multiplying by $\Omega^{d-1}$ overall, giving

$$
\mathbb{I}=2 \pi^{2} i \Omega^{d-1}\left[-\left(\frac{2}{\epsilon}-\gamma_{\mathrm{E}}+\ln (4 \pi)\right)-\frac{\ln (2 i \Omega a)+1}{i \Omega a}-\ln (\Omega a)+3 \ln (2 \pi)+\frac{i \pi}{2}\right],
$$

which can also be written as

$$
\mathbb{I}=2 \pi^{2}(i \Omega)^{d-1}\left[-\left(\frac{2}{\epsilon}-\gamma_{\mathrm{E}}+\ln (4 \pi)\right)-\frac{\ln (2 i \Omega a)+1}{i \Omega a}-\ln (i \Omega a)+3 \ln (2 \pi)\right],
$$

\section{G.9 Generalization to include $M<0$}

Let $\mathbb{I}(M, \Omega)$ represent $\mathbb{I}$ as a function of $M$ and $\Omega$. From the definition of $\mathbb{I}$ by (G.1) and (G.2), one can see that

$$
\mathbb{I}(-M, \Omega)=\left[\mathbb{I}\left(M, \Omega^{*}\right)\right]^{*} .
$$

This relation tells us how to get the result for negative $M$ from the result (G.46) for positive $M$. We can implement the relation by rewriting (G.46) as

$$
\mathbb{I}=2 \pi^{2}(i \bar{\Omega})^{d-1}\left[-\left(\frac{2}{\epsilon}-\gamma_{\mathrm{E}}+\ln (4 \pi)\right)-\frac{\ln (2 i \bar{\Omega} a)+1}{i \bar{\Omega} a}-\ln (i \bar{\Omega} a)+3 \ln (2 \pi)\right]
$$

with $\bar{\Omega} \equiv \Omega \operatorname{sgn} M$ as in (4.32). This is the result that was quoted in (4.31).

\section{H Technical points concerning front-end transformations}

\section{H.1 Branch cuts for the transformation $(\mathrm{k}) \rightarrow(\mathrm{r})$}

The result given by eqs. (A.41) and (A.42) for diagram (k) contains a term

$$
-\frac{N_{\mathrm{f}} \alpha_{\mathrm{EM}}^{2}}{6 \pi^{2}} P_{e \rightarrow e}\left(x_{e}\right) 2 \operatorname{Re}\left\{i \Omega_{\mathrm{i}} \operatorname{sgn}\left(M_{\mathrm{i}}\right) \ln \left(\frac{\mu^{2}}{\left(1-x_{e}\right) E \Omega_{\mathrm{i}} \operatorname{sgn} M_{\mathrm{i}}}\right)\right\} .
$$

Under a combined front-end and back-end trasnformation, this term transforms the same way as (4.42). [See the comment after (4.42) to understand the relation to (4.40).] Under this transformation, $\Omega_{\mathrm{i}} \rightarrow\left(\Omega_{0}^{\gamma \rightarrow e \bar{e}}\right)^{*}$ and $\operatorname{sgn}\left(M_{\mathrm{i}}\right) \rightarrow-1$. (H.1) transforms to

$$
\begin{aligned}
&-\frac{N_{\mathrm{f}} \alpha_{\mathrm{EM}}^{2}}{6 \pi^{2}} P_{\gamma \rightarrow e}\left(y_{e}\right) 2 \operatorname{Re}\{\left.-i\left(\Omega_{0}^{\gamma \rightarrow e \bar{e}}\right)^{*} \ln \left(\frac{\mu^{2}}{-E\left(\Omega_{0}^{\gamma \rightarrow e \bar{e}}\right)^{*}}\right)\right\} \\
&=-\frac{N_{\mathrm{f}} \alpha_{\mathrm{EM}}^{2}}{6 \pi^{2}} P_{\gamma \rightarrow e}\left(y_{e}\right) 2 \operatorname{Re}\left\{i \Omega_{0}^{\gamma \rightarrow e \bar{e}} \ln \left(\frac{\mu^{2}}{-E \Omega_{0}^{\gamma \rightarrow e \bar{e}}}\right)\right\} .
\end{aligned}
$$

This result may be confusing depending on whether one thinks that the minus sign inside the logarithm represents $e^{i \pi}$ or $e^{-i \pi}$. In general, we try to write expressions so that the relevant branch cut for logarithms is along the negative real axis. Since $\Omega_{0}^{\gamma \rightarrow e \bar{e}} \propto \sqrt{-i}$ 
(which should be interpreted as $e^{-i \pi / 4}$ in LPM formulas), that means that (H.2) above is the same as

$$
-\frac{N_{\mathrm{f}} \alpha_{\mathrm{EM}}^{2}}{6 \pi^{2}} P_{\gamma \rightarrow e}\left(y_{e}\right) 2 \operatorname{Re}\left\{i \Omega_{0}^{\gamma \rightarrow e \bar{e}}\left[\ln \left(\frac{\mu^{2}}{E \Omega_{0}^{\gamma \rightarrow e \bar{e}}}\right)-i \pi\right]\right\}
$$

which is how we have written it in (A.52).

To understand why this is the correct interpretation of the logarithm in (H.2), and the origin of why there is a $-i \pi$ term in (H.3) for diagram (r) that is not in (H.1) for diagram $(\mathrm{k})$, we need to step back to an earlier stage of the derivation. The important difference revolves around the overall factor of $1 / i^{d}$ in (F.30) for the $x y y \bar{x}(\mathrm{k})$ diagram. This factor can be traced back to the small- $\Delta t$ expression for the 4-particle (effectively 2-particle) propagator in ACI3 (4.9) [9], which can be viewed as the product of a free propagator for $\boldsymbol{C}_{34}$ times a free propagator for $\boldsymbol{C}_{12}$,

$$
\left\langle\boldsymbol{C}_{34}, \boldsymbol{C}_{12}, \Delta t \mid \boldsymbol{C}_{34}^{\prime}, \boldsymbol{C}_{12}^{\prime}, 0\right\rangle \simeq\left(\frac{\mathfrak{M}_{34}}{2 \pi i \Delta t}\right)^{d / 2} e^{i\left|\boldsymbol{C}_{34}-\boldsymbol{C}_{34}^{\prime}\right|^{2} / 2 \Delta t} \times\left(\frac{\mathfrak{M}_{12}}{2 \pi i \Delta t}\right)^{d / 2} e^{i\left|\boldsymbol{C}_{12}-\boldsymbol{C}_{12}^{\prime}\right|^{2} / 2 \Delta t},
$$

with

$$
\begin{aligned}
& \mathfrak{M}_{34}=x_{3} x_{4}\left(x_{3}+x_{4}\right) E, \\
& \mathfrak{M}_{12}=x_{1} x_{2}\left(x_{1}+x_{2}\right) E=-x_{1} x_{2}\left(x_{3}+x_{4}\right) E
\end{aligned}
$$

For the $x y y \bar{x}$ diagram, $\left(x_{1}, x_{2}, x_{3}, x_{4}\right)=\left(-1, y_{e}, 1-x_{e}-y_{e}, x_{e}\right)$, which means that both of the masses $\left(\mathfrak{M}_{34}, \mathfrak{M}_{12}\right)$ are positive. The total phase associated with the exponential prefactors in (H.4) is then

$$
\left(\frac{1}{i}\right)^{d / 2} \times\left(\frac{1}{i}\right)^{d / 2}=\left(\frac{1}{i}\right)^{d} \quad \text { for diagram }(\mathrm{k}) .
$$

This is the origin of the overall $i^{-d}$ in (F.30). The reason $\left(\mathfrak{M}_{34}, \mathfrak{M}_{12}\right)$ given by (H.5) were positive was that for the 4-particle portion of the evolution in figure $8 \mathrm{k}$ there is only one line which is in the conjugate amplitude $\left(x_{1}<0\right)$ and the other three are all in the amplitude $\left(x_{2}, x_{3}, x_{4}>0\right)$. In contrast, for the 4-particle portion of the evolution in figure 9 r, there are two lines in the conjugate amplitude (two of the $x_{i}<0$ ) and two in the amplitude (the other two $\left.x_{i}>0\right)$, and so one of $\left(\mathfrak{M}_{34}, \mathfrak{M}_{12}\right)$ will be positive and one will be negative. The analog of (H.6) is then

$$
\left(\frac{1}{i}\right)^{d / 2} \times\left(-\frac{1}{i}\right)^{d / 2}=1 \quad \text { for diagram }(\mathrm{r}) .
$$

One can consider (H.7) as the product of an ordinary free propagator times the conjugate of an ordinary free propagator, for which the overall phases cancel.

In consequence, if we had done a direct calculation of (r) [instead of using our frontend trick], there would have been a relative factor of $i^{d}$ between our calculations of $(\mathrm{k})$ and our calculations of (r). When multiplied by the $2 / \epsilon$ divergence of those calculations, 
like in (F.45), this leads to a relative additive term $\pm i \pi$ between the finite terms in (F.45) and their analog for $(\mathrm{r})$. So, ultimately, the origin of the $-i \pi$ difference between the form of (H.1) and (H.3) is that in figure 8k the earliest splitting and the photon self-energy loop both happen in the amplitude whereas in figure 9r, one happens in the amplitude and the other in the conjugate amplitude.

\section{H.2 Front-end transformations in $d=2-\epsilon$ dimensions}

The front-end transformation rules given in the main text were for $d=2$. On a diagram by diagram basis, there are some subtleties conerning overall normalization when $d=2-\epsilon$, which we now discuss, but they do not affect any of the results in this paper.

\section{H.2.1 Transformation of leading-order rates}

The easiest way to find the necessary modifications is to consider the transformation (4.42) that related leading-order rates for $e \rightarrow \gamma e$ and $\gamma \rightarrow e \bar{e}$. In $d=2-\epsilon$ dimensions, the $x \bar{x}$ diagram for $e \rightarrow \gamma e$ is given by (F.44) as

$$
\left[\frac{d \Gamma}{d x_{e}}\right]_{x \bar{x}}^{e \rightarrow \gamma e}=-\frac{\mu^{\epsilon} \alpha_{\mathrm{EM}} d}{8 \pi} P_{e \rightarrow e}^{(d)}\left(x_{e}\right) \mathrm{B}\left(\frac{1}{2}+\frac{d}{4},-\frac{d}{4}\right)\left(\frac{2 \pi}{M_{\mathrm{i}} \bar{\Omega}_{\mathrm{i}}}\right)^{\epsilon / 2} i \bar{\Omega}_{\mathrm{i}}
$$

with $\bar{\Omega}_{\mathrm{i}}=\Omega_{\mathrm{i}} \operatorname{sgn}\left(M_{\mathrm{i}}\right)$ and $M_{\mathrm{i}}=x_{e}\left(1-x_{e}\right) E$. If we take a front-end and back-end transformation, as shown in figure 42, we should get the conjugate of the analogous $\gamma \rightarrow e \bar{e}$ result, which is

$$
\left[\frac{d \Gamma}{d y_{e}}\right]_{y \bar{y}}^{\gamma \rightarrow e \bar{e}}=-\frac{N_{\mathrm{f}} \mu^{\epsilon} \alpha_{\mathrm{EM}} d}{8 \pi} P_{\gamma \rightarrow e}^{(d)}\left(y_{e}\right) \mathrm{B}\left(\frac{1}{2}+\frac{d}{4},-\frac{d}{4}\right)\left(\frac{2 \pi}{M_{\gamma \rightarrow e \bar{e}} \overline{\Omega_{\gamma \rightarrow e \bar{e}}}}\right)^{\epsilon / 2} i \bar{\Omega}_{\gamma \rightarrow e \bar{e}}
$$

with $M_{\gamma \rightarrow e \bar{e}}=y_{e}\left(1-y_{e}\right) E$. In addition to the $d$-dimensional splitting function of (F.34), we also need ${ }^{52}$

$$
P_{e \rightarrow e}^{(d)}(z)=\frac{1+z^{2}}{1-z}-\frac{\epsilon}{2}(1-z) .
$$

One can check that the transformation (4.42) does not do the job to relate (H.8) to (H.9). To get it to work, one must modify the overall power of $1-y_{e}$ in $(4.42)$ to $\left(1-y_{e}\right)^{1-\epsilon}$. One also needs to account for the fact that the rate $e \rightarrow \gamma e$ is averaged over electron helicities and summed over photon helicities, whereas the rate for $\gamma \rightarrow e \bar{e}$ is the opposite. In dimensional regularization, the number $\mathcal{N}_{\gamma}$ of initial photon helicities is $2-\epsilon$, but the number of initial electron helicites $\mathcal{N}_{e}$ is fixed by the convention $\operatorname{tr}\left(\mathbb{1}_{\text {Dirac }}\right) \equiv 4$ to be exactly 2 . We need to account for this difference by including a factor $\mathcal{N}_{e} / \mathcal{N}_{\gamma}$ in our transformation. One can check already at the level of the DGLAP splitting functions (F.34) and (H.10) that

$$
P_{\gamma \rightarrow e}^{(d)}(z)=(1-z) \frac{\mathcal{N}_{e}}{\mathcal{N}_{\gamma}} P_{e \rightarrow e}^{(d)}\left(\frac{-z}{1-z}\right) .
$$

Using that, one finds that the $d=2-\epsilon$ generalization of (4.42) is

$$
2 \operatorname{Re}\left[\frac{d \Gamma}{d y_{e}}\right]_{y \bar{y}}^{\gamma \rightarrow e \bar{e}}=2 N_{\mathrm{f}}\left(1-y_{e}\right)^{1-\epsilon} \frac{\mathcal{N}_{e}}{\mathcal{N}_{\gamma}} \operatorname{Re}\left\{\left[\frac{d \Gamma}{d x_{e}}\right]_{x \bar{x}}^{e \rightarrow \gamma e} \text { with }\left(x_{e}, E\right) \rightarrow\left(\frac{-y_{e}}{1-y_{e}},\left(1-y_{e}\right) E\right)\right\} .
$$

\footnotetext{
${ }^{52}$ See, for example, eq. (14) of ref. [36] with similar conversions as in our earlier footnote 46.
} 


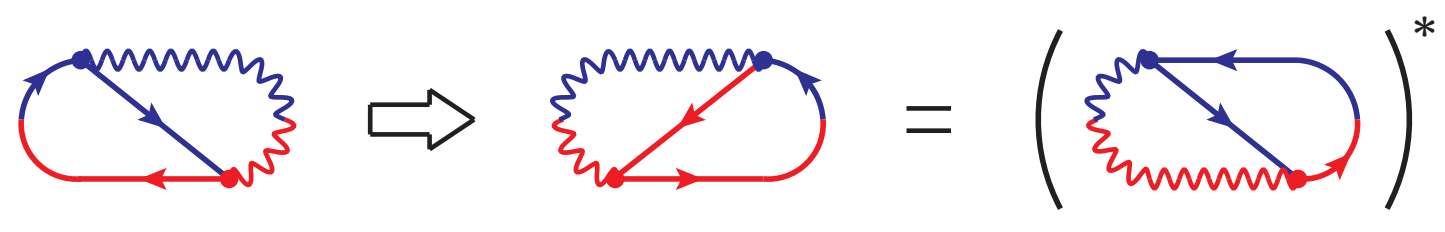

Figure 42. The conversion of the leading-order diagram $x \bar{x}$ for $e \rightarrow \gamma e$ via front-end and back-end transformation into the conjugate of the leading-order diagram $y \bar{y}$ for $\gamma \rightarrow e \bar{e}$.

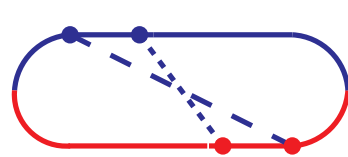

$x y \bar{y} \bar{x}$

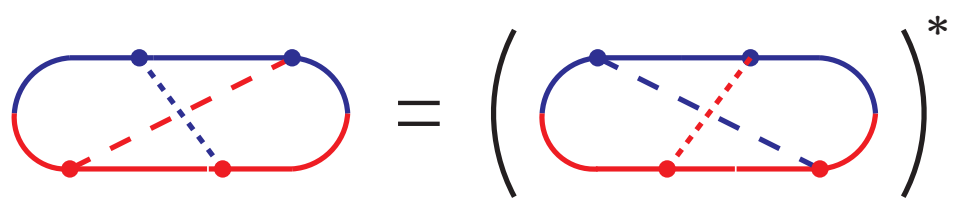

$(x \bar{y} y \bar{x})^{*}$

Figure 43. The conversion of the QCD diagram $x y \bar{y} \bar{x}$ for $g \rightarrow g g g$ via front-end and back-end transformation into the conjugate of the diagram $x \bar{y} y \bar{x}$ for $g \rightarrow g g g$. Long and short dashed lines represent final-state gluons with longitudinal momentum fractions $x$ and $y$ respectively.

\section{H.2.2 Transformation of NLO rates}

To get the transformation law for NLO rates, we can just use the obsevation after (4.42) that one can interpret $\left[d \Gamma / d x_{e} d y_{e}\right]_{x \bar{x}}^{e \rightarrow \gamma e} \equiv\left[d \Gamma / d x_{e}\right]_{x \bar{x}}^{e \rightarrow \gamma e} \delta\left(y_{e}\right)$ in the leading-order rate formula. The $d=2-\epsilon$ analog of (4.40) will then be

$$
\begin{array}{r}
2 \operatorname{Re}\left[\frac{d \Gamma}{d y_{e}}\right]_{(\mathrm{r})}=2 N_{\mathrm{f}}\left(1-y_{e}\right)^{-\epsilon} \frac{\mathcal{N}_{e}}{\mathcal{N}_{\gamma}} \operatorname{Re} \int_{0}^{1} d x_{e}\left\{\left[\frac{d \Gamma}{d x_{e} d y_{e}}\right]_{(\mathrm{k})}\right. \text { with } \\
\left.\left(x_{e}, y_{e}, E\right) \rightarrow\left(\frac{-y_{e}}{1-y_{e}}, \frac{x_{e}}{1-y_{e}},\left(1-y_{e}\right) E\right)\right\} .
\end{array}
$$

However, we do not need this in this paper. Because the additional factor of $\left(1-y_{e}\right)^{-\epsilon} \mathcal{N}_{e} / \mathcal{N}_{\gamma}$ we needed to add to the $d=2$ transformation laws was the same for both the leading-order and NLO rates, it can be factored out when transforming the final renormalized rate (F.46). Since the renormalized rate is finite, we can then set $\epsilon=0$, and so the additional factor is just 1 and makes no difference there. We would only need the additional factor if we wanted to individually transform divergent diagrams.

\section{H.2.3 A check: the QCD $x y \bar{y} \bar{x}$ and $x \bar{y} y \bar{x}$ diagrams}

Part of the reason we went through this discussion is so that we could make an explicit check of front-end transformations using previous results [7,9] for QCD diagrams. In particular, a front-end plus back-end trasnformation should relate the two $g \rightarrow g g g$ interference diagrams depicted in figure 43. These diagrams are each UV divergent (particular time-orderings of tree-level diagrams can be UV divergent even though the sum of all time-orderings is not), and so one must use the $d=2-\epsilon$ version of the transformation. Here, the initial particles 
are always gluons, so that transformation law is simply

$$
2 \operatorname{Re}\left[\frac{\Delta d \Gamma}{d x d y}\right]_{x \bar{y} y \bar{x}}=2(1-y)^{-\epsilon}\left\{\left[\frac{\Delta d \Gamma}{d x d y}\right]_{x y \bar{y} \bar{x}} \text { with }(x, y, E) \rightarrow\left(\frac{-y}{1-y}, \frac{x}{1-y},(1-y) E\right)\right\} .
$$

Using formulas for $x y \bar{y} \bar{x}$ and $x \bar{y} y \bar{x}$ from AI1 [7] and ACI3 [9], we have checked that the above transformation indeed works. ${ }^{53}$

Open Access. This article is distributed under the terms of the Creative Commons Attribution License (CC-BY 4.0), which permits any use, distribution and reproduction in any medium, provided the original author(s) and source are credited.

\section{References}

[1] L.D. Landau and I. Pomeranchuk, Limits of applicability of the theory of bremsstrahlung electrons and pair production at high-energies (in Russian), Dokl. Akad. Nauk Ser. Fiz. 92 (1953) 535 [INSPIRE].

[2] L.D. Landau and I. Pomeranchuk, Electron cascade process at very high energies (in Russian), Dokl. Akad. Nauk Ser. Fiz. 92 (1953) 735 [InSPIRE].

[3] A.B. Migdal, Bremsstrahlung and pair production in condensed media at high-energies, Phys. Rev. 103 (1956) 1811 [INSPIRE].

[4] J.-P. Blaizot and Y. Mehtar-Tani, Renormalization of the jet-quenching parameter, Nucl. Phys. A 929 (2014) 202 [arXiv:1403.2323] [InSPIRE].

[5] E. Iancu, The non-linear evolution of jet quenching, JHEP 10 (2014) 095 [arXiv:1403.1996] [INSPIRE].

[6] B. Wu, Radiative energy loss and radiative $p_{\perp}$-broadening of high-energy partons in $Q C D$ matter, JHEP 12 (2014) 081 [arXiv:1408.5459] [INSPIRE].

[7] P. Arnold and S. Iqbal, The LPM effect in sequential bremsstrahlung, JHEP 04 (2015) 070 [Erratum ibid. 09 (2016) 072] [arXiv: 1501.04964] [INSPIRE].

[8] P. Arnold, H.-C. Chang and S. Iqbal, The LPM effect in sequential bremsstrahlung 2: factorization, JHEP 09 (2016) 078 [arXiv: 1605.07624] [INSPIRE].

[9] P. Arnold, H.-C. Chang and S. Iqbal, The LPM effect in sequential bremsstrahlung: dimensional regularization, JHEP 10 (2016) 100 [arXiv:1606.08853] [INSPIRE].

[10] P. Arnold, H.-C. Chang and S. Iqbal, The LPM effect in sequential bremsstrahlung: 4-gluon vertices, JHEP 10 (2016) 124 [arXiv: 1608.05718] [INSPIRE].

\footnotetext{
${ }^{53}$ Specifically, $x y \bar{y} \bar{x}$ consists of (i) a divergent "pole contribution" given by ACI3 (4.36) and (4.37) plus (ii) the non-pole piece given by AI1 (5.45) integrated just over $\Delta t>a$. The corresponding contributions to $x \bar{y} y \bar{x}$ are then obtained from the transformations described in AI1 section VI.A. To get the overall sign in (H.14) above, one must add absolute value signs to the various longitudinal momentum fractions in the formulas AI1 (4.39) for $(\alpha, \beta, \gamma)$, just like we had to add absolute value signs to the $(\bar{\alpha}, \bar{\beta}, \bar{\gamma})$ of this paper in order to be able to implement front-end transformations (see footnote 38). Since it is only $x$ (and not $y$ or $1-x$ or $1-y$ or $1-x-y$ ) that negates under the transformation in (H.14), it is sufficient to just replace the factors of $x$ and $x^{3}$ by $|x|$ and $|x|^{3}$ in AI (4.39).
} 
[11] G.P. Lepage and S.J. Brodsky, Exclusive Processes in Perturbative Quantum Chromodynamics, Phys. Rev. D 22 (1980) 2157 [InSPIRE].

[12] S.J. Brodsky and G.P. Lepage, Exclusive Processes in Quantum Chromodynamics, Adv. Ser. Direct. High Energy Phys. 5 (1989) 93 [INSPIRE].

[13] S.J. Brodsky, H.-C. Pauli and S.S. Pinsky, Quantum chromodynamics and other field theories on the light cone, Phys. Rept. 301 (1998) 299 [hep-ph/9705477] [INSPIRE].

[14] Y.V. Kovchegov and E. Levin, Quantum chromodynamics at high energy, Camb. Monogr. Part. Phys. Nucl. Phys. Cosmol. 33 (2012) [errata available at https://www.physics.ohio-state.edu/ yuri/typos.pdf] [INSPIRE].

[15] L. Mantovani, B. Pasquini, X. Xiong and A. Bacchetta, Revisiting the equivalence of light-front and covariant QED in the light-cone gauge, Phys. Rev. D 94 (2016) 116005 [arXiv: 1609.00746] [INSPIRE].

[16] P. Arnold, S. Iqbal and T. Rase, Strong-vs. weak-coupling pictures of jet quenching: a dry run using QED, arXiv:1810.06578 [INSPIRE].

[17] G.D. Moore, Transport coefficients in large $N_{f}$ gauge theory: Testing hard thermal loops, JHEP 05 (2001) 039 [hep-ph/0104121] [INSPIRE].

[18] A. Ipp, G.D. Moore and A. Rebhan, Comment on and erratum to 'Pressure of hot QCD at large $N_{f}^{\prime}$, JHEP 01 (2003) 037 [hep-ph/0301057] [INSPIRE].

[19] G.D. Moore, Pressure of hot QCD at large $N_{f}$, JHEP 10 (2002) 055 [hep-ph/0209190] [INSPIRE].

[20] B.G. Zakharov, Fully quantum treatment of the Landau-Pomeranchuk-Migdal effect in QED and QCD, JETP Lett. 63 (1996) 952 [hep-ph/9607440] [INSPIRE].

[21] B.G. Zakharov, Radiative energy loss of high-energy quarks in finite size nuclear matter and quark-gluon plasma, JETP Lett. 65 (1997) 615 [hep-ph/9704255] [INSPIRE].

$[22]$ G. Beuf, Dipole factorization for DIS at NLO: Loop correction to the $\gamma_{T, L}^{*} \rightarrow q \bar{q}$ light-front wave functions, Phys. Rev. D 94 (2016) 054016 [arXiv: 1606.00777] [INSPIRE].

[23] G. Beuf, Dipole factorization for DIS at NLO: Combining the $q \bar{q}$ and $q \bar{q} g$ contributions, Phys. Rev. D 96 (2017) 074033 [arXiv:1708.06557] [INSPIRE].

[24] T. Lappi and R. Paatelainen, The one loop gluon emission light cone wave function, Annals Phys. 379 (2017) 34 [arXiv:1611.00497] [INSPIRE].

[25] H. Hänninen, T. Lappi and R. Paatelainen, One-loop corrections to light cone wave functions: the dipole picture DIS cross section, Annals Phys. 393 (2018) 358 [arXiv: 1711.08207] [INSPIRE].

[26] Particle Data Group collaboration, C. Patrignani et al., Review of Particle Physics, Chin. Phys. C 40 (2016) 100001 [INSPIRE].

[27] R. Baier, Y.L. Dokshitzer, A.H. Mueller, S. Peigne and D. Schiff, The Landau-Pomeranchuk-Migdal effect in QED, Nucl. Phys. B 478 (1996) 577 [hep-ph/9604327] [INSPIRE].

[28] R. Baier, Y.L. Dokshitzer, A.H. Mueller, S. Peigne and D. Schiff, Radiative energy loss of high-energy quarks and gluons in a finite volume quark-gluon plasma, Nucl. Phys. B 483 (1997) 291 [hep-ph/9607355] [INSPIRE]. 
[29] R. Baier, Y.L. Dokshitzer, A.H. Mueller, S. Peigne and D. Schiff, Radiative energy loss and $p_{\perp}$-broadening of high-energy partons in nuclei, Nucl. Phys. B 484 (1997) 265 [hep-ph/9608322] [INSPIRE].

[30] P.B. Arnold and W. Xiao, High-energy jet quenching in weakly-coupled quark-gluon plasmas, Phys. Rev. D 78 (2008) 125008 [arXiv:0810.1026] [InSPIRE].

[31] S. Caron-Huot, O(g) plasma effects in jet quenching, Phys. Rev. D 79 (2009) 065039 [arXiv: 0811.1603] [INSPIRE].

[32] A. Peshier, QCD running coupling and collisional jet quenching, J. Phys. G 35 (2008) 044028 [INSPIRE].

[33] P.B. Arnold, Simple Formula for High-Energy Gluon Bremsstrahlung in a Finite, Expanding Medium, Phys. Rev. D 79 (2009) 065025 [arXiv:0808.2767] [INSPIRE].

[34] T. Liou, A.H. Mueller and B. Wu, Radiative $p_{\perp}$-broadening of high-energy quarks and gluons in QCD matter, Nucl. Phys. A 916 (2013) 102 [arXiv:1304.7677] [INSPIRE].

[35] S. Klein, Suppression of Bremsstrahlung and pair production due to environmental factors, Rev. Mod. Phys. 71 (1999) 1501 [hep-ph/9802442] [InSPIRE].

[36] S. Catani and M. Grazzini, Collinear factorization and splitting functions for next-to-next-to-leading order QCD calculations, Phys. Lett. B 446 (1999) 143 [hep-ph/9810389] [INSPIRE].

[37] G. 't Hooft and M.J.G. Veltman, Regularization and Renormalization of Gauge Fields, Nucl. Phys. B 44 (1972) 189 [InSPIRE].

[38] I.S. Gradshteyn and I.M. Ryzhik, Table of Integrals, Series, and Products, 4th corrected and enlarged edition, Academic Press (1980). 